\title{
Ward identities and combinatorics of rainbow tensor models
}

\author{
H. Itoyama, ${ }^{a, b}$ A. Mironov ${ }^{c, d, e, f}$ and A. Morozov ${ }^{d, e, f}$ \\ ${ }^{a}$ Department of Mathematics and Physics, Graduate School of Science, Osaka City University, \\ 3-3-138, Sugimoto, Sumiyoshi-ku, Osaka, 558-8585, Japan \\ ${ }^{b}$ Osaka City University Advanced Mathematical Institute (OCAMI), \\ 3-3-138, Sugimoto, Sumiyoshi-ku, Osaka, 558-8585, Japan \\ ${ }^{c}$ I.E.Tamm Theory Department, Lebedev Physics Institute, \\ Leninsky prospect, 53, Moscow 119991, Russia \\ ${ }^{d}$ ITEP, \\ B. Cheremushkinskaya, 25, Moscow, 117259, Russia \\ e Institute for Information Transmission Problems, \\ Bolshoy Karetny per. 19, build.1, Moscow 127051 Russia \\ ${ }^{f}$ National Research Nuclear University MEPhI, \\ Moscow 115409, Russia \\ E-mail: itoyama@sci.osaka-cu.ac.jp, mironov@lpi.ru, morozov@itep.ru
}

ABSTRACT: We discuss the notion of renormalization group ( $R G$ ) completion of nonGaussian Lagrangians and its treatment within the framework of Bogoliubov-Zimmermann theory in application to the matrix and tensor models. With the example of the simplest non-trivial RGB tensor theory (Aristotelian rainbow), we introduce a few methods, which allow one to connect calculations in the tensor models to those in the matrix models. As a byproduct, we obtain some new factorization formulas and sum rules for the Gaussian correlators in the Hermitian and complex matrix theories, square and rectangular. These sum rules describe correlators as solutions to finite linear systems, which are much simpler than the bilinear Hirota equations and the infinite Virasoro recursion. Search for such relations can be a way to solving the tensor models, where an explicit integrability is still obscure.

Keywords: Matrix Models, Random Systems, Holography and condensed matter physics (AdS/CMT), Lattice Models of Gravity

ARXIV EPRINT: 1704.08648 


\section{Contents}

1 Introduction 1

2 Combinatorics of matrix models: old results and new claims 4

2.1 Hermitian matrix model 5

2.1.1 Partition function and Ward identities 5

2.1.2 The simplest averages from Virasoro recursion 5

2.1.3 Pictorial representation 6

2.1.4 Genus expansion, spectral curve and topological recursion $\quad 8$

2.1.5 W-representation 9

2.1.6 Alternative generating functions and their Fourier transform 9

2.1.7 Kontsevich representation of Hermitian model 12

$\begin{array}{lll}2.1 .8 & \text { Integrability } & 12\end{array}$

2.2 Complex matrix model of [114, 170-172] 13

$\begin{array}{lll}2.3 & \text { Rectangular complex matrix model } & 15\end{array}$

$\begin{array}{lll}\text { 2.3.1 Partition function and Ward identities } & 15\end{array}$

$\begin{array}{ll}\text { 2.3.2 The simplest averages from Virasoro recursion } & 15\end{array}$

$\begin{array}{lll}2.3 .3 & W \text {-representation } & 18\end{array}$

$\begin{array}{lll}3 & \text { On the universal structure of Virasoro-like constraints } & 19\end{array}$

$\begin{array}{lll}3.1 & \text { Keystone operators and their RG-descendants } & 19\end{array}$

3.2 Tree operators as the base of RG-complete set 21

$\begin{array}{lll}3.3 & \text { The simplest recursions } & 24\end{array}$

$\begin{array}{lll}3.4 & \text { BZ exponential and rooted trees } & 25\end{array}$

3.5 The Bogoliubov-Zimmermann tensor model 27

3.6 Archetypical/universal Virasoro constraint 28

$\begin{array}{lll}3.7 & \text { Relation to Feynman diagrams } & 29\end{array}$

$\begin{array}{lll}3.8 & \text { BZ resolvents } & 29\end{array}$

$\begin{array}{lll}3.9 & \text { The message } & 29\end{array}$

4 RG-closed tensor generalization of the complex matrix model 30

$\begin{array}{ll}4.1 \text { Partition function } & 30\end{array}$

4.2 Notation: two types of diagrams 31

4.3 Ordinary Virasoro constraints for an oversimplified tensor model 32

4.4 Spectral curve as the leading term of the genus expansion 33

4.5 Examples of averages 33

4.6 Recursions 34

4.7 Integrability properties: does Virasoro imply integrability? 36

$\begin{array}{lll}\text { 4.7.1 } W \text {-representation } & 36\end{array}$

$\begin{array}{lll}4.8 & \text { The message } & 36\end{array}$ 
5 A non-trivial RG-closed extension of the Aristotelian tensor model

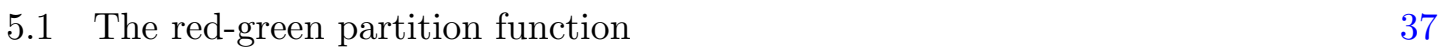

$\begin{array}{lll}5.2 & \text { Hierarachy/tower of Virasoro-like constraints } & 38\end{array}$

5.3 Gaussian averages in the Aristotelian model by direct computation 41

$\begin{array}{lll}\text { 5.3.1 Red rings and green rings } & 41\end{array}$

5.3.2 Reductions at $N=1 \quad 41$

5.3.3 Red and green rings together 41

5.3.4 Direct evaluation of $\left\langle\mathcal{K}_{2,2}\right\rangle \quad 43$

5.3.5 Some other Gaussian averages $\left\langle\mathcal{K}_{m, n}\right\rangle$ and their reductions 44

$\begin{array}{lll}\text { 5.3.6 Wheel operators } & 47\end{array}$

5.3.7 Examples of recursion checks 47

5.4 On RG completion of the Aristotelian model 49

5.5 The structure of RG-complete set of operators 51

5.6 Towards Ward identities $\quad 52$

5.7 Towards solvability of tensor models $\quad 54$

6 Conclusion $\quad 56$

\section{Introduction}

The key problem in study of interactions in quantum field theory (QFT) is an identification of RG-complete actions which contain all local operators that can be generated by the action of renormalization group. Such theories possess rich Ward identities associated with change of integration variables in the functional integral, which can be alternatively associated with the diffeomorphisms in the space of couplings. In the conventional QFT, this technique is known as the Bogoliubov-Zimmermann (BZ) renormalization theory [1-5]. In matrix models, it reduces to the theory of generalized Virasoro/W-constraints [6-10] and to the formalism of check operators [11-15]. In the both cases, the story consists of two steps:

(1) The original interaction (operator), which we will call the keystone operator, is complemented by its tree descendants (see section 3 below for an explanation of this central notion). These are often not all independent and, in the theories possessing the space-time, where one often distinguishes between the UV and IR RG-completeness, many of them can be non-local and neglected in the study of, say, the UV renormalization group. Inclusion of the tree descendants makes the theory quasiclassically complete and reduces the Ward relation (diffeomorphism) symmetry to representation theory of the tree composition algebra in graph theory. The old-fashioned renormalizability relevant for identification of the RG stable low-energy theories like the Standard Model implies that only a finite number of tree operators is generated, but, in generic string/M-theory operative at the Planck scales, this restriction is not necessarily imposed. Of course, it is never imposed in the theory of matrix and tensor models along the lines of [16-24], where the space-time degrees of freedom are not present at all, and there is no difference between local and non-local operators. 
(2) Unfortunately, besides the tree operators, there are also loop operators, and in the study of Ward identities a la [6-10] they emerge from the Jacobians of change of the integration variables. Taming of loop operators is the main problem in the search for the RG-complete theories. In fact, some of loop operators reduce to tree operators, e.g. all the loop operators made from the planar or melonic diagrams in matrix and tensor models respectively. Sometimes, all the other loop operators are algebraically expressed through the tree operators. The famous example is the Hermitian matrix model, where the tree and loop operators are respectively the single- and multi-trace operators, the latter being just the products of single-trace ones.

In one-matrix models, we are accustomed to a very simple description of all possible "gauge-invariant" operators: they are just products of traces, $\prod_{i} \operatorname{tr} M^{k_{i}}$. However, already for the two-matrix models the situation changes drastically: even the single-trace operators $\operatorname{Tr}\left(\prod_{i} A^{k_{i}} B^{l_{i}}\right)$ are labeled by words and are difficult to enumerate in any efficient way. In result, while, in the first case, it is easy to define generating functions: they are products of, say, the resolvents $\operatorname{Tr} \frac{1}{z-M}$, i.e. the functions of a single variable $z$, in the second case, the counterpart of the resolvent is far more involved and depends on infinitely many variables $x_{i}$ and $y_{i}$ : $\operatorname{Tr} \prod_{i} \frac{1}{x_{i}-A} \frac{1}{y_{i}-B}$. In the multi-matrix and tensor models, the set of operators allowed by symmetry becomes more and more involved. However, the point is that most of these enumerable operators are in fact a kind of alien to the original one. If the keystone operator was, say $\operatorname{Tr}(A B A B)$, then neither $\operatorname{Tr}\left(A B^{2} A B\right)$ nor $\operatorname{Tr}\left(A B^{2} A^{2} B\right)$ would ever arise as (RG) time goes, if they were not present from the very beginning. This does not mean that they have vanishing correlators, this means that they have vanishing averages at all times provided they were not present in the initial state. In other words, such operators can be excluded from consideration by a superselection rule (like superpositions of neutron and proton, it can also deserve reminding that the gauge invariance is also present in YangMills theories in the sense of the same rule: if the initial state was gauge invariant, then such are all the states in the course of its evolution).

In fact, the RG-completion is a slightly weaker statement: it admits not only the operators directly present in the keystone evolution operator, but also their further descendants. Allowed are all operators emerging in the course of multi-evolutions (many time variables) associated with all the descendants of the keystone operator. Still, the operators like $\operatorname{Tr}\left(A B^{2} A^{2} B\right)$ and their more sophisticated counterparts would never emerge. In variance with $\operatorname{Tr}\left(A B^{2} A B\right)$, they are not forbidden by any explicit symmetry of the model. What forbids them is a hidden symmetry. In this case, it is the possibility of generalization [26-28] from the square to rectangular matrices $A$ and $B$, where such operators simply can not exist, and, therefore, do not appear among the descendants; in result they can not appear even if one makes the matrices square again, only the generically allowed $\operatorname{Tr}(A B)^{k}$ can emerge. Revealing and exploiting such symmetries is the main idea of studying the rainbow tensor models, their enhanced symmetries make properties of the RG-completeness much simpler and explicit. In [25], we already mentioned one example of this kind: in the rainbow models melonic are the only existing among the planar diagrams, and this is what explains this their dominance at large $N$ in all tensor models. However, the examples are 
not at all exhausted by this one, and we exploit the power of the rainbow models more in the present text.

Thus, in a particular model, not all the operators permitted by symmetry necessarily arise in the course of evolution and the ones which arise can form a smaller set, much easier enumerated than one could expect. Moreover, when considered from the right perspective, these sets come with their own hierarchy, which also provides a useful approach to the RG-evolution problem. As already mentioned, only the tree descendants of the keystone operators matter quasiclassically and already this reduces the set, when the keystone is carefully selected, especially in the theories with high symmetry like the rainbow tensor models of [25]. The question is what happens at the loop operator level. There are at least three options to consider:

First: all loop operators are algebraic functions of the tree ones like it happens in the one-matrix model;

Second: some loop operators are independent of the tree ones, but they also form a comprehensible subset, which can be just added to the action, while all the rest of emerging operators are expressed through them;

Third: this happens at the level of averages in certain limits, as an analogy of the factorization of multi-trace operators at large $N$, only this time this can be used to formulate a model that is RG-complete in the limit (the ordinary matrix model is RG-complete irrespective of any limits and factorizations).

The long-awaited surge in attention [29]-[57] to the tensor models [58-76]-[95] allows one to begin a systematic investigation of these problems. They did not receive enough attention within the matrix model context, because the multi-matrix models [96-99] were long considered as rather exotic objects, but, in the tensor case, the issue arises already in the indisputably beautiful examples. The questions are what are the extended partition functions of these models, where the full sets of symmetry-allowed operators are enormous and practically innumerable? Can we restrict our consideration to some nicer subsets? How do we distinguish between allowed subsets, and what makes them closed and the model consistent?

As already reminded at the beginning of this introduction, in the conventional renormalizable quantum field theory, the requirements come from the unitarity of the regularized evolution operator and are guaranteed by the application of the Bogoliubov-Zimmermann procedure. The question is what substitutes it (or how it looks) in generic string theory, i.e. within the context of generic tensor categories and, to begin with, of generic matrix and tensor models. This question was addressed in [100] in association with the work by A. Connes and D. Kreimer [101-107] (which describes the Bogoliubov-Zimmermann formulas in terms of the Hopf algebra of Feynman diagrams). The true motivation was, however, somewhat broader and included also the search for the QFT reformulation of the problems of non-linear algebra [108-111]. In the present paper, we discuss further steps towards constructing the renormalization group (RG) complete models and the RG-closed sets of 
operators. We adopt a simplest option for the definition of the complete models: to request that they possess a sufficiently rich set of the Ward identities, which can make them potentially integrable (in a sense which still needs to be defined). In other words, one can begin from the search of the tensor models, which are as close in their solvability to the Hermitian matrix model as possible.

Investigating this problem, we actually discovered a previously unknown feature of matrix models: they possess additional, linear and finite, relations between Gaussian averages, which allow one to find them explicitly and provide a tremendously simple character expansion for the extended partition function, with coefficients made from the dimensions of representations of GL $(N)$. This is the long-awaited property explaining what lies at the intersection of $\mathrm{KP}$ integrability and Virasoro constraints and what is so peculiar for the matrix-model $\tau$-functions. More important in the present context is that this is a simple property, for which one can straightforwardly look in the tensor models, once one manages to perform explicit calculations, and at the very end of this paper we provide some initial evidence in favor of its existence.

We begin in section 2 by reminding the basics of matrix model theory from [16-24] and some of more recent papers. We also report the discovery of new relations and explicit formulas for arbitrary Gaussian averages and extended (coupling/time-dependent) partition functions. Then, in section 3 we remind the basics of BZ theory in the formalism of [100], best suited for applications to the matrix and tensor models. In the remaining part of the paper, we discuss two simple examples of the rainbow-type tensor models. The "red" model in section 4 trivially reduces to a rectangular complex matrix model, but another, "red-green" model in section 5 (which actually has three colorings and can be naturally called RGB or Aristotelian) exhibits interesting deviations from it, which are already peculiar for tensor models. Further generalization to the most interesting case with the tetrahedron-like interaction remains as a next natural step to make.

\section{Combinatorics of matrix models: old results and new claims}

The most interesting tensor models are the far-going generalizations of the eigenvalue matrix models, where everything needs to be re-analyzed: expressions for the averages, recurrent relations between them, their solutions provided by the $W$-representations, the genus expansions, the spectral curves and the AMM/EO-topological recursions, and their interpretations in terms of integrable systems, the $\mathrm{KP} /$ Toda and Hurwitz $\tau$-functions. Still, there are artificially designed tensor models, which deviate from the matrix case in a minimal way, with different directions of deviation, while preserving one or another of the matrix model properties. Thus, their study is useful not only for the initial steps in the tensor model theory, but also for clarifying the origins and universality of particular structures, revealed in the matrix model studies. This section provides a basis for such an analysis, which is attempted in the remaining part of the paper.

The first question to address in any model is evaluation of the correlators (averages of various operators). This can be done either by direct calculation or by using the Ward identities. We mostly concentrate on the interplay between these two, with the 
$W$-representations and integrability mentioned only in passing. Instead, we suggest to define the Gaussian correlators from very simple and finite sets of linear equations, which efficiently substitute both the Virasoro constraints and integrability.

\subsection{Hermitian matrix model}

\subsubsection{Partition function and Ward identities}

The model is associated with the integral over the $N \times N$ matrix $M$

$$
Z_{H}=\int d M \exp \left(-\frac{\mu}{2} \operatorname{Tr} M^{2}\right)
$$

where the measure is induced by the norm $\|\delta M\|^{2}=\operatorname{Tr}(\delta M)^{2}$ and the Ward identities are the usual Virasoro constraints [6-10] for the extended partition function

$$
\mathcal{Z}_{H}\{t\}=\int d M \exp \left(-\frac{\mu}{2} \operatorname{Tr} M^{2}+\sum_{k} t_{k} \operatorname{Tr} M^{k}\right)
$$

that is,

$$
\hat{L}_{n}^{H} \mathcal{Z}_{H}=\left(-\mu \frac{\partial}{\partial t_{n+2}}+\sum k t_{k} \frac{\partial}{\partial t_{k+n}}+\sum_{a=1}^{n-1} \frac{\partial^{2}}{\partial t_{a} \partial t_{n-a}}+2 N \frac{\partial}{\partial t_{n}}+N^{2} \delta_{n, 0}\right) \mathcal{Z}_{H}, \quad n \geq-1
$$

(one often simplifies the formula by introducing the time $t_{0}$ with the additional constraint $\frac{\partial \mathcal{Z}_{H}}{\partial t_{0}}=N \mathcal{Z}_{H}$ but a similar counterpart of this trick is not known for the rectangular and tensor models).

\subsubsection{The simplest averages from Virasoro recursion}

The correlators

$$
\mathcal{O}_{\Lambda}=\left\langle\prod_{i=1}^{l_{\Lambda}} \operatorname{Tr} M^{\lambda_{i}}\right\rangle=\left.\frac{1}{\mathcal{Z}}\left(\prod_{i} \frac{\partial}{\partial t_{\lambda_{i}}}\right) \mathcal{Z}\right|_{t=0}
$$

are naturally labeled by the Young diagrams $\Lambda$ with $l_{\Lambda}$ rows,

$$
\Lambda=\left\{\lambda_{1} \geq \lambda_{2} \geq \ldots \geq \lambda_{l_{\Lambda}}>0\right\}
$$

They can be recursively restored by solving the Virasoro constraints and their $t$-derivatives.

The first steps of the recursion are:

$$
\begin{array}{ccc}
\hat{L}_{0}^{H} \mathcal{Z}_{H}=0 & \Longrightarrow & \mu\left\langle\operatorname{Tr} M^{2}\right\rangle=N^{2} \\
\frac{\partial}{\partial t_{1}} \hat{L}_{-1}^{H} \mathcal{Z}_{H}=0 & \Longrightarrow & \mu\langle\operatorname{Tr} M \operatorname{Tr} M\rangle=N \\
\hat{L}_{2}^{H} \mathcal{Z}_{H}=0 & \Longrightarrow \mu\left\langle\operatorname{Tr} M^{4}\right\rangle=2 N\left\langle\operatorname{Tr} M^{2}\right\rangle+\langle\operatorname{Tr} M \operatorname{Tr} M\rangle=\frac{2 N^{3}+N}{\mu}
\end{array}
$$

see [112] for continuation of the list. The parameter $\mu$ is kept in these formulas to identify the "direction" of the recursion: every recursion step adds an extra power of $\mu$ in the denominator. 


\subsubsection{Pictorial representation}

One rarely uses pictures in discussing general features of the matrix models: an analytical language is developed well enough for writing easily readable formulas. Things are still very different in the tensor models, where at this stage we need to express many ideas pictorially. Because of this, we now do the same in the familiar matrix model case, this can facilitate an understanding of pictures in the next sections. We use the same colorings as there. In the rainbow tensor models, there are several $\mathrm{U}(N)$ gauge groups and fields are charged with respect to different collections of these groups, thus, colored are the types of indices in the fields and fields themselves, we call this coloring as multi-coloring, preserving the word "color" for the values of indices inside the fundamental representation of the particular gauge group. The multi-coloring could also be called "flavor", or, even better, "techni-flavor", but we decided to avoid this terminology. In the Hermitian matrix model, only one type of coloring remains, we choose it red. Multi-coloring is also reduced to a single specie: a pair of red lines.

The operators $\operatorname{Tr} M^{k}$ can be depicted as polygons with $k$ angles. In particular, the "keystone" operator $\operatorname{Tr} M^{3}$ and its first descendant $\operatorname{Tr} M^{4}$ are:
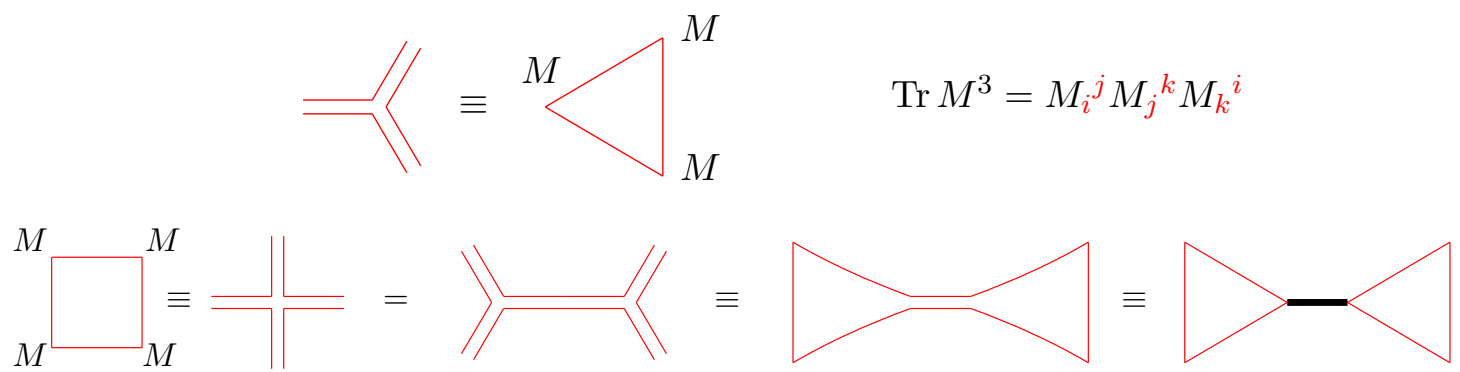

We remind that the lines in the matrix model pictures are used to describe the contraction of indices. Note the interplay between the double and single red lines. The thick black line denotes the tensor $\delta_{i^{\prime}}^{i} j_{j}^{j^{\prime}}$ or the action of the operator $\operatorname{Tr}\left(\frac{\partial}{\partial M} \otimes \frac{\partial}{\partial M}\right)$, which plays the role of the propagator in the matrix model. The identity in the picture is manifestation of the relation

$$
\operatorname{Tr} M^{4}=\frac{1}{9} \frac{\partial \operatorname{Tr} M^{3}}{\partial M_{i}^{j}} \frac{\partial \operatorname{Tr} M^{3}}{\partial M_{i}^{j}}
$$

with a combinatorial coefficient omitted. The parameter $\mu^{-1}$ can be easily included or omitted, as one prefers. In generic QFT, the propagator contains also a "propagating" (space-time dependent) factor, which makes the "composite" operator non-local, still a similar formalism is useful to describe the convolution of indices, it is enough to omit $\mu$, with all the derivatives it can contain. This blowing up of interaction vertices (operators) in Feynman diagrams does not make too much sense in theories with the matrix-valued fields, like the Yang-Mills theories, however, in the tensor models, where the indices are much less under control, this formalism becomes very useful.

Clearly, all the operators $\prod_{i=1}^{L+1} \operatorname{Tr} M^{k_{i}}$ can be depicted in this blown-up formalism as triangles connected by thick black lines, moreover, in many different ways. The singletrace operators with $L+1=1$ emerge in this way from the trees, while $L$ is the number 
of loops in the graph with black edges. In other words, in this formalism of describing the keystone descendants, the single-traces are the tree operators and the multi-traces are loop operators. This is a formulation which can be easily extended from matrices to tensors, where the notion of trace is not very relevant.

Another element of the formalism is an operator $M^{m}$ with open ends (with no trace), we denote it by the thick red line (vector):

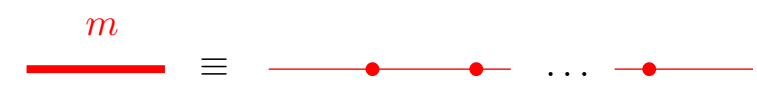

Let us note that the colored lines throughout the text are associated not with elements of the Feynman technique, but depict operators, or, more exactly, their color structure: how the concrete operator is constructed from the colored fields. In particular, the loop colored lines (without external ends) denote the invariant operators.

One can use just the same thick line with another label $z$ to denote a sum over $m$, for example $(z-M)^{-1}$, then its trace, resolvent will be depicted as a thick red circle. One can consider also the traces like $\operatorname{Tr} e^{s M}$ etc. In the next sections, we use only the thick lines and circles with indices $m$.

An important feature of the thick red line is that the thick black propagator can be attached to its interior, moreover we have identities like

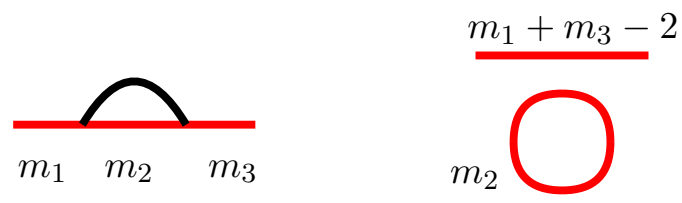

which can be described by the formula

$$
\begin{aligned}
& \left(\operatorname{Tr} \frac{\partial}{\partial M} \frac{\partial}{\partial M}\right)\left[\left(M^{m_{1}-1}\right)_{i}{ }^{m} M_{m}{ }^{k}\left(M^{m_{2}}\right)_{k}{ }^{n} M_{n}{ }^{l}\left(M^{m_{3}-1}\right)_{l}{ }^{j}\right]= \\
& \quad=\left(M^{m_{1}-1}\right)_{i}{ }^{l}\left(M^{m_{2}}\right)_{k}{ }^{k}\left(M^{m_{3}-1}\right)_{l}{ }^{j}=\left(M^{m_{1}+m_{3}-2}\right)_{i}{ }^{j}\left(M^{m_{2}}\right)_{k}{ }^{k}
\end{aligned}
$$

and overbrackets denote the Wick pairing, i.e. the concrete field in the expression that is differentiated.

In application to a thick red circle, this identity converts the thick black propagator into an operator cutting one circle into two pieces, then the recursion relation underlying the Virasoro identities is just

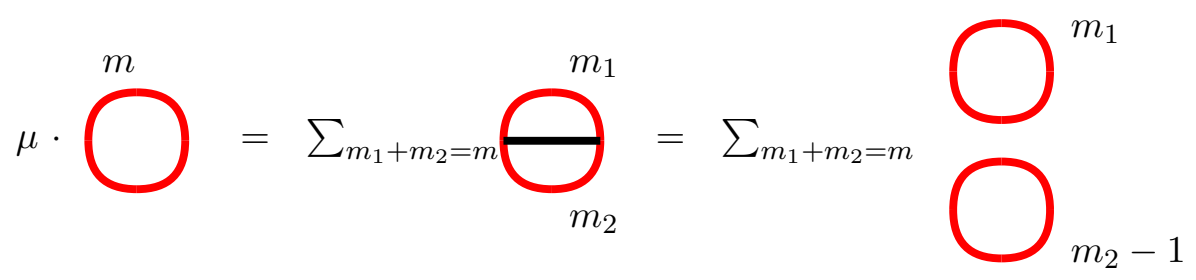




\subsubsection{Genus expansion, spectral curve and topological recursion}

The simplest way to deal with the Ward identities like (2.3) is to rewrite them in the form of the loop equation

$$
-\mu z \rho^{(1)}(z)+\mu N+\rho^{(1)}(z)^{2}+\rho^{(2)}(z, z)+\left[V^{\prime}(z) \rho^{(1)}(z)\right]_{-}=0
$$

for (multi-)resolvents (connected components of multi-trace correlators)

$$
\rho^{(n)}\left(z_{1}, \ldots, z_{n}\right)=\hat{\nabla}_{z_{1}} \ldots \hat{\nabla}_{z_{n}} \log \mathcal{Z}
$$

Note that we further often refer to $\rho^{(1)}(z)$ as just to $\rho(z)$. In these formulas, $[\ldots]_{-}$means projection onto the negative powers of $z$,

$$
V(z)=\sum_{k} t_{k} z^{k} \quad \text { and } \quad \hat{\nabla}_{z}=\sum_{k \geq 0} \frac{1}{z^{k+1}} \frac{\partial}{\partial z_{k}}
$$

and we defined the derivative $\frac{\partial Z}{\partial t_{0}} \equiv N Z$.

One can take the loop equation (2.9) at all $t_{k}=0$,

$$
-\mu z \rho^{(1)}(z)+\mu N+\rho^{(1)}(z)^{2}+\rho^{(2)}(z, z)=0
$$

then, apply the operator $\hat{\nabla}_{z}$ to $(2.9)$ and again put all $t_{k}=0$, which includes $\rho^{(3)}(z)$ etc. This gives a kind of Bogoliubov chain relations. In order to construct an effective recursion, one has to go further and introduce also a parameter $g$ of the quasiclassical, or genus expansion via rescaling $t_{k} \rightarrow \frac{1}{\hbar} t_{k}, \log Z \rightarrow \frac{1}{\hbar^{2}} \log Z, N \rightarrow \frac{1}{\hbar} N$. Now one can consider the planar limit (leading order in $\hbar$ ). This reduces (2.12) to an algebraic equation

$$
-\mu z \rho_{0}(z)+\mu N+\rho_{0}(z)^{2}=0
$$

which solution is

$$
\rho_{0}(z)=\frac{\mu}{2}\left(z-\sqrt{z^{2}-\frac{4 N}{\mu}}\right)
$$

The imaginary part of $\rho_{0}(z)$ describes the density of the eigenvalues in the matrix model, and is equal to

$$
y(z)=\mu \sqrt{z^{2}-\frac{4 N}{\mu}}
$$

This is the notorious semi-circle distribution [113], which satisfies the equation

$$
y(z)^{2}=\mu^{2}\left(z^{2}-\frac{4 N}{\mu}\right)
$$

and is called the spectral curve (in this concrete case, it is a sphere). 
Dealing with the loop equations, one achieves at least two goals:

- One can promote recursions between particular correlators to those between their particular generating functions, the best known example is the genus expansion with the AMM/EO topological recursion [11-13, 112, 114-118] between contributions of different genera $\rho^{(n \mid g)}$ of multi-resolvents,

$$
\rho^{(n)}\left(z_{1}, \ldots, z_{n}\right)=\sum_{g=0}^{\infty} \hbar^{1-g} \rho_{g}^{(n)}\left(z_{1}, \ldots, z_{n}\right)
$$

which are actually meromorphic poly-differentials on the spectral curve, which is an equation for the ordinary resolvent at genus zero. In the particular case of Hermitian model, the Gaussian planar resolvent (2.14) as a function of $1 / \mu$ is a generating function of the Catalan numbers. As pointed out in [115-118], in abstract form, the topological recursion is applicable to arbitrary families of Riemann surfaces and thus works in many examples, where a matrix-model realization is not yet discovered.

- One can shift any time-variable $t_{k} \longrightarrow T_{k}+t_{k}$, not only $t_{2} \longrightarrow t_{2}-\frac{\mu}{2}$, and to consider $t$ expansions of $\mathcal{Z}$ around non-Gaussian points parameterized by the superpotentials $W(z)=\sum T_{k} z^{k}$. This leads to the theory of Dijkraaf-Vafa phases $[11-13,112,119-$ 135], which depend drastically on the power of the polynomial $W(z)$.

Despite best studied, the loop equation/resolvent approach has a serious drawback: even in the simplest case of Hermitian model at the Gaussian point it does not produce a full answer for correlators: no multi-resolvent $\rho^{(n)}$ was so far calculated in closed form, only particular components $\rho^{(n \mid g)}$ of their genus expansions are known. To solve this kind of problems, one can proceed at least in two other ways: look at the $W$-representations of $\mathcal{Z}(t)$ and look at less naive (model-dependent) generating functions rather than at the ordinary (universal) resolvents.

\subsubsection{W-representation}

$W$-representation [136-140] provides a simple "dual" formula for $\mathcal{Z}\{t\}$, expressing it through differentiation rather than integration:

$$
\mathcal{Z}_{H}\{t\}=e^{\frac{1}{2 \mu} \hat{W}_{H}} e^{N t_{0}}
$$

where the relevant cut-and-join operator is a $(-2)$-harmonic of the simplest operator from a big family studied in [141, 142]:

$$
\hat{W}_{H}=\sum_{a, b}\left(a b t_{a} t_{b} \frac{\partial}{\partial t_{a+b-2}}+(a+b+2) t_{a+b+2} \frac{\partial^{2}}{\partial t_{a} \partial t_{b}}\right)
$$

\subsubsection{Alternative generating functions and their Fourier transform}

Instead of resolvents, one can consider various other generating functions of the correlators in the matrix model. For instance, one can use the Wilson loops [143-148]

$$
\operatorname{Tr} e^{s M}=\sum_{k} \frac{s^{k}}{k !} \operatorname{Tr} M^{k}
$$


and, especially interesting, the Harer-Zagier generating function [149-153]

$$
\sum_{k=0}^{\infty} \frac{z^{k}}{(2 k-1) ! !}\left\langle\operatorname{Tr}_{N \times N} M^{2 k}\right\rangle=\frac{\mu}{2 z}\left(\left(\frac{\mu+z}{\mu-z}\right)^{N}-1\right)
$$

and its Fourier transform (FT) in the matrix size [112]

$$
\sum_{N, k} \frac{\lambda^{N} z^{k}}{(2 k-1) ! !}\left\langle\operatorname{Tr}_{N \times N} M^{2 k}\right\rangle=\frac{\lambda}{(1-\lambda)(1-\lambda-(1+\lambda) z / \mu)}
$$

This FT generating function leads to far more explicit expressions for matrix model averages. In result,

$$
\mathrm{FT}_{[k]}=\sum_{\lambda} \lambda^{N}\left\langle\operatorname{Tr}_{N \times N} M^{2 k}\right\rangle=\frac{\lambda(1+\lambda)^{k}}{\mu^{k}(1-\lambda)^{k+2}} \cdot(2 k-1) ! !
$$

which one can easily use with the help of binomial expansion

$$
\frac{1}{(1-\lambda)^{k+2}}=\sum_{N} \lambda^{N} \frac{(N+k+1) !}{(k+1) ! N !}
$$

In particular,

$$
\begin{aligned}
\mu\left\langle\operatorname{Tr} M^{2}\right\rangle & =N^{2}, & \mu^{2}\left\langle\operatorname{Tr} M^{4}\right\rangle & =2 N^{3}+N, \\
\mu^{3}\left\langle\operatorname{Tr} M^{6}\right\rangle & =5 N^{4}+10 N^{2}, & \mu^{4}\left\langle\operatorname{Tr} M^{8}\right\rangle & =14 N^{5}+70 N^{3}+21 N, \\
\mu^{5}\left\langle\operatorname{Tr} M^{10}\right\rangle & =42 N^{6}+420 N^{4}+483 N^{2}, & \mu^{6}\left\langle\operatorname{Tr} M^{12}\right\rangle & =132 N^{7}+2310 N^{5}+6468 N^{3}+1485 N, \ldots
\end{aligned}
$$

Similar generating functions for the exact Gaussian correlators are also available from [152, 153] for the double- and triple-trace averages $\left\langle\operatorname{Tr} M^{k_{1}} \operatorname{Tr} M^{k_{2}}\right\rangle$ and $\left\langle\operatorname{Tr} M^{k_{1}} \operatorname{Tr} M^{k_{2}} \operatorname{Tr} M^{k_{3}}\right\rangle$.

These Gaussian correlators satisfy amusing sum rules, for example:

$$
\begin{gathered}
\pm\left\langle\operatorname{Tr} M^{2}\right\rangle+\left\langle(\operatorname{Tr} M)^{2}\right\rangle= \pm N(N \pm 1), \\
\pm 6\left\langle\operatorname{Tr} M^{4}\right\rangle+8\left\langle\operatorname{Tr} M^{3} \operatorname{Tr} M\right\rangle+3\left\langle\left(\operatorname{Tr} M^{2}\right)^{2}\right\rangle \pm 6\left\langle\operatorname{Tr} M^{2}(\operatorname{Tr} M)^{2}\right\rangle+\left\langle(\operatorname{Tr} M)^{4}\right\rangle \\
=3 N(N \pm 1)(N \pm 2)(N \pm 3), \\
\mp 2\left\langle\operatorname{Tr} M^{4}\right\rangle-\left\langle\left(\operatorname{Tr} M^{2}\right)^{2}\right\rangle \pm 2\left\langle\operatorname{Tr} M^{2}(\operatorname{Tr} M)^{2}\right\rangle+\left\langle(\operatorname{Tr} M)^{4}\right\rangle=-(N+1) N(N-1)(N \pm 2), \\
-4\left\langle\operatorname{Tr} M^{3} \operatorname{Tr} M\right\rangle+3\left\langle\left(\operatorname{Tr} M^{2}\right)^{2}\right\rangle+\left\langle(\operatorname{Tr} M)^{4}\right\rangle=3(N+1) N^{2}(N-1),
\end{gathered}
$$

The coefficients at the l.h.s. are actually the properly normalized symmetric group characters $\varphi_{R}(\Lambda)$ from $[141,142]$, so that, in general, the sum rules are

$$
\left.\frac{1}{d_{R} \cdot \mathcal{Z}_{H}} \cdot \chi_{R}\left\{\frac{1}{n} \frac{\partial}{\partial t_{n}}\right\} \mathcal{Z}_{H}\right|_{t=0}=\sum_{\Lambda \vdash|R|} \varphi_{R}(\Lambda) \cdot \mathcal{O}_{\Lambda}=c_{R} \cdot \frac{D_{R}(N)}{d_{R}}
$$

for all Young diagrams $R$ of even size (number of boxes) $|R|$. Here $\chi_{R}$ and $D_{R}(N)$ are respectively the Schur polynomials and the dimensions of representation $R$ of the linear 
group $\mathrm{GL}(N)$, the factor $d_{R}=\chi_{R}\left(t_{n}=\delta_{n, 1}\right)$ is the dimension of representation $R$ of the symmetric group $S_{|R|}$ divided by $|R|$ ! [154]. The coefficients $c_{R}$ are occasionally equal to $\varphi_{R}([2]), \varphi_{R}([2,2])$ and $\varphi_{R}([2,2,2])$ for $|R|=2,|R|=4$ and $|R|=6$ respectively, with an obvious implication for the general case. These sum rules allow one to express all averages $\mathcal{O}_{\Lambda}$ through those for the single-line Young diagrams $\mathcal{O}_{[|\Lambda|]}$, which are fully described by (2.23). They also provide a simple formula for the character expansion of the partition function:

$$
\begin{aligned}
\mathcal{Z}_{H}\{t\} & =\sum_{\text {even size } R} \varphi_{R}(\underbrace{[2, \ldots, 2]}_{|R| / 2}) \cdot D_{R}(N) \cdot \chi_{R}\{t\} \\
& =\sum_{\text {even size } R} \varphi_{R}(\underbrace{[2, \ldots, 2]}_{|R| / 2}) \cdot \chi_{R}\left\{t_{n}=\frac{N}{n}\right\} \cdot \chi_{R}\{t\}
\end{aligned}
$$

Existence of simple formulas like (2.21), and thus of their far-going generalization (2.27) for the Hermitian matrix model seems to reflect $[112,152,153]$ its $\mathrm{KP} /$ Toda integrability [16-24, 155-157], i.e. a somewhat deeper structure than just the Ward identities. In particular, integrability requires the coefficients $c_{R}$ to be made from the Casimir exponentials [158-161]. Like the Virasoro recursion these relations are linear in correlators and like the Hirota bilinear identities they preserve the grading: hence, they combine the advantages of these both. They are sufficient to obtain any Gaussian correlator: using the orthogonality relation

$$
\sum_{R} d_{R}^{2} \varphi_{R}(\Lambda) \varphi_{R}\left(\Lambda^{\prime}\right)=\frac{1}{z_{\Lambda}} \delta_{\Lambda, \Lambda^{\prime}}
$$

where $z_{\Lambda}$ is the standard symmetric factor of the Young diagram (order of the automorphism) [154], one can obtain from (2.27)

$$
\mathcal{O}_{\Lambda}=z_{\Lambda} \sum_{R \vdash|\Lambda|} c_{R} \cdot d_{R} \cdot D_{R}(N) \cdot \varphi_{R}(\Lambda)=\sum_{R \vdash|\Lambda|} \varphi_{R}(\underbrace{[2, \ldots, 2]}_{|R| / 2}) \cdot D_{R}(N) \cdot \psi_{R}(\Lambda)
$$

where $\psi_{R}(\Lambda)=z_{\Lambda} d_{R} \varphi_{R}(\Lambda)$ are the conventionally normalized characters [154] called by the command $C h i(R, \Lambda)$ in MAPLE. Since all the quantities $\varphi_{R}(\Lambda), d_{R}, z_{\Lambda}$ and $D_{R}(N)$ are well-known from the basic representation theory, these formulas provide a long-lookedfor complete perturbative solution to the Hermitian matrix model (perturbative means that it is still restricted to the Gaussian point, while the non-perturbative analysis of the Dijkraaf-Vafa phases still requires the use of Virasoro constraints a la [112]). It would be very interesting to find a counterpart of this phenomenon and these formulas for the tensor model, see section 5.7 below for a first step in this direction.

One more important property of the Gaussian correlators is much simpler: it just reflects the fact that $-\frac{\mu}{2}$ is a background value of the time variable $t_{2}$. Because of this, the very special kind of averages gets factorized:

$$
\begin{aligned}
\mathcal{O}_{\left[\Lambda, 2^{n}\right]} & =\left.\mu^{N^{2} / 2}\left(\frac{\partial}{\partial t_{2}}\right)^{n}\left(\frac{1}{\mu^{N^{2} / 2}} \mathcal{O}_{\Lambda}\right)\right|_{t=0}=\mu^{N^{2} / 2}\left(-2 \frac{\partial}{\partial \mu}\right)^{n}\left(\frac{1}{\mu^{N^{2} / 2}} \mathcal{O}_{\Lambda}\right) \\
& =\frac{1}{\mu^{n}} \mathcal{O}_{\Lambda} \cdot \prod_{i=0}^{n-1}\left(N^{2}+|\Lambda|+2 i\right)
\end{aligned}
$$

where we took into account the obvious fact that $\mathcal{O}_{\Lambda} \sim \mu^{-|\Lambda| / 2}$. 


\subsubsection{Kontsevich representation of Hermitian model}

Integrability of matrix model (see section 2.1.8) inspires a highly non-trivial transform of the partition functions called Miwa transformation which expresses the time-variables $t_{k}$ in terms of a matrix-valued background field $A$,

$$
t_{k}=-\frac{1}{k} \operatorname{Tr} A^{-k}
$$

At the particular values of the matrix size $N$, this would be a counterpart of the topological locus in Chern-Simons/knot theory [164-168] describing particular distinguished slices in the space of time-variables, however, it should be considered at arbitrary large $N$. For the Hermitian model in the Gaussian phase, this transformation was first described in $[157,169]$ (see also [114]) and looks like:

$$
\left.\mathcal{Z}_{H}\right|_{t_{k}=-\frac{1}{k} \operatorname{Tr} A^{-k}}=\mathcal{Z}_{\mathrm{GHK}}(A) \sim \int d M \operatorname{det} M^{N} \exp \left(-\frac{1}{2 \mu} \operatorname{Tr} M^{2}-i \operatorname{Tr} M A\right)
$$

The Virasoro constraints are now straightforward consequences of the equations of motion, and the cut-and-join operator generating the $W$-representation (2.18) is just a Laplacian

$$
\hat{W}=\operatorname{tr}\left(\frac{\partial^{2}}{\partial A^{2}}-\frac{N}{A}\right)
$$

in the Miwa variables.

A counterpart of the Kontsevich transform in non-Gaussian DV phases has been never worked out.

\subsubsection{Integrability}

The Gaussian Hermitian matrix model describes an integrable system: the partition function (2.2) is a $\tau$-function of the (forced) Toda chain [155-157] (see [162] for a discussion of integrability in non-Gaussian phases). This means that it satisfies the equation w.r.t. the size of matrix $N$,

$$
\mathcal{Z}_{H}\{t \mid N\} \frac{\partial^{2} \mathcal{Z}_{H}\{t \mid N\}}{\partial t_{1}^{2}}-\left(\frac{\partial \mathcal{Z}_{H}\{t \mid N\}}{\partial t_{1}^{2}}\right)^{2}=\mathcal{Z}_{H}\{t \mid N+1\} \mathcal{Z}_{H}\{t \mid N-1\}
$$

or, in terms of the resolvent,

$$
\rho(z \mid N+1)+\rho(z \mid N-1)-2 \rho(z \mid N)=\frac{1}{N} \partial_{z}^{2} \rho(z \mid N)
$$

The latter equation is easily transformed into formulas (2.21)-(2.23), [112, Part IV], see also [152, 153]. Explicit solution of the Toda chain that describes this concrete matrix model is distinguished either by the string equation [155-157], or by the determinant representation explicitly

$$
\mathcal{Z}_{H}\{t \mid N\}=\operatorname{det}_{0 \leq i, j \leq N} C_{i+j}, \quad C_{k} \equiv \int d x x^{k} \exp \left(-\frac{\mu x^{2}}{2}+\sum_{k} t_{k} x^{k}\right)
$$

However, there is another possibility to relate the Gaussian Hermitian model with the Toda lattice $\tau$-function [163]: one can note that it is equal to the concrete model from the big family of Hurwitz partition functions considered in [140],

$$
Z_{(k, m)}\left\{\mu, N_{1}, \ldots, N_{m} \mid t^{(i)}\right\}=\sum_{R} \mu^{-|R|} d_{R}^{2-k-m}\left(\prod_{i=1}^{k} \chi_{R}\left\{t^{(i)}\right\}\right)\left(\prod_{i=1}^{m} D_{R}\left(N_{i}\right)\right)
$$


Indeed, from (2.28) one observes that the Gaussian Hermitian model is equivalent to the Toda lattice $\tau$-function $Z_{(2,1)}$ at some special point in the second set of time variables $\{\bar{t}\}$ :

$$
\mathcal{Z}_{H}\{t \mid N\}=Z_{(2,1)}\left\{\mu, N \mid \bar{t}_{k}=\frac{1}{2} \delta_{k, 2}, t_{k}\right\}
$$

This explains emerging the strange group character factor $\varphi_{R}(\underbrace{[2, \ldots, 2]}_{|R| / 2})$ in $(2.30)$ and the sum over the Young diagrams of even sizes, and can be directly obtained from the matrix model of the Kontsevich type describing $Z_{(2,1)}$, [140, eq. (64)]: one can note that this matrix model reduces to (2.33) upon putting $\bar{t}_{k}=\frac{1}{2} \delta_{k, 2}$, the latter being equivalent to the Gaussian Hermitian model, as was explained in the previous subsection.

\subsection{Complex matrix model of $[114,170-172]$}

The complex matrix model is an integral over complex $N \times N$ matrices $M$ with the Gaussian kinetic term $\operatorname{Tr} M M^{\dagger}=\operatorname{Tr} M^{\dagger} M$. In what follows, we often denote $M^{\dagger} \equiv \bar{M}$ to simplify formulas, i.e. $\bar{M}$ denotes the Hermitian, not just complex conjugation. The kinetic term can be perturbed in two essentially different ways:

$$
\int d^{2} M \exp \left(-\mu \operatorname{Tr} M M^{\dagger}+\operatorname{Tr} M^{m}+\operatorname{Tr} M^{\dagger^{m}}\right)
$$

with $m=3$ or $m=4$ or

$$
\int d^{2} M \exp \left(-\mu \operatorname{Tr} M M^{\dagger}+\operatorname{Tr}\left(M M^{\dagger}\right)^{2}\right)
$$

These different choices of keystone operators lead to RG-completions with essentially different symmetries: $\mathrm{U}(N)$ and $\mathrm{U}(N) \otimes \mathrm{U}(N)$. In the latter case, "gauge" invariant are only the operators made from $\operatorname{Tr}(M \bar{M})^{k}$, while, in the former case, one can take traces of arbitrary matrix products. We mostly consider the latter model with the extended symmetry in this paper, though the former one is also used in some examples.

The extended partition function with $\mathrm{U}(N) \otimes \mathrm{U}(N)$ symmetry is defined by the integral

$$
\mathcal{Z}_{C}\{t\}=\int d^{2} M \exp \left(-\mu \operatorname{Tr} M M^{\dagger}+\sum_{k} t_{k} \operatorname{Tr}\left(M M^{\dagger}\right)^{k}\right)
$$

Its significant difference from the Hermitian model is that the odd powers of $M$ can not appear in the action, and, therefore, this time the $\hat{L}_{-1}$ constraint is absent:

$$
\left(-\mu \frac{\partial}{\partial t_{n+1}}+\sum_{k} k t_{k} \frac{\partial}{\partial t_{k+n}}+\sum_{a=1}^{n-1} \frac{\partial^{2}}{\partial t_{a} \partial t_{n-a}}+2 N \cdot\left(1-\delta_{n, 0}\right) \frac{\partial}{\partial t_{n}}+N^{2} \cdot \delta_{n, 0}\right) \mathcal{Z}_{C}\{t\}=0, n \geq 0
$$


Instead, the first term with the coefficient $\mu$ contains $t_{n+1}$ rather than $t_{n+2}$, what makes the recursive extraction of correlators well defined. The first few examples are (they are particular cases of (2.44) below with $\alpha=2 N$ and $\beta=N^{2}$ ):

$\mathcal{O}_{[1]}=\frac{N^{2}}{\mu}$

$\mathcal{O}_{[2]}=\frac{2 N^{3}}{\mu^{2}} \quad \mathcal{O}_{[1,1]}=\frac{N^{2}\left(N^{2}+1\right)}{\mu^{2}}$

$\mathcal{O}_{[3]}=\frac{N^{2}\left(5 N^{2}+1\right)}{\mu^{3}} \quad \mathcal{O}_{[2,1]}=\frac{2 N^{3}\left(N^{2}+2\right)}{\mu^{3}} \quad \mathcal{O}_{[1,1,1]}=\frac{N^{2}\left(N^{2}+1\right)\left(N^{2}+2\right)}{\mu^{3}}$

$\mathcal{O}_{[4]}=\frac{2 N^{3}\left(7 N^{2}+5\right)}{\mu^{4}} \quad \mathcal{O}_{[3,1]}=\frac{N^{2}\left(N^{2}+3\right)\left(5 N^{2}+1\right)}{\mu^{4}} \quad \mathcal{O}_{[2,2]}=\frac{N^{2}\left(2 N^{4}+9 N^{2}+1\right)}{\mu^{4}}$

$\mathcal{O}_{[2,1,1]}=\frac{2 N^{3}\left(N^{2}+2\right)\left(N^{2}+3\right)}{\mu^{4}} \quad \mathcal{O}_{[1,1,1,1]}=\frac{N^{2}\left(N^{2}+1\right)\left(N^{2}+2\right)\left(N^{2}+3\right)}{\mu^{4}}$

$\mathcal{O}_{[5]}=\frac{2 N^{2}\left(21 N^{4}+35 N^{2}+4\right)}{\mu^{5}} \quad \mathcal{O}_{[4,1]}=\frac{2 N^{3}\left(N^{2}+4\right)\left(7 N^{2}+5\right)}{\mu^{5}} \quad \mathcal{O}_{[3,2]}=\frac{2 N^{3}\left(9 N^{4}+37 N^{2}+18\right)}{\mu^{5}}$

$\mathcal{O}_{[3,1,1]}=\frac{N^{2}\left(5 N^{2}+1\right)\left(N^{2}+3\right)\left(N^{2}+4\right)}{\mu^{5}} \quad \mathcal{O}_{[2,2,1]}=\frac{2 N^{2}\left(11 N^{2}+1\right)\left(N^{2}+4\right)}{\mu^{5}}$

$\mathcal{O}_{[2,1,1,1]}=\frac{2 N^{3}\left(N^{2}+2\right)\left(N^{2}+3\right)\left(N^{2}+4\right)}{\mu^{5}} \quad \mathcal{O}_{[1,1,1,1,1]}=\frac{N^{2}\left(N^{2}+1\right)\left(N^{2}+2\right)\left(N^{2}+3\right)\left(N^{2}+4\right)}{\mu^{5}}$

So far the generating functions like (2.21) and (2.23) were not available for these correlators. Moreover, in this respect the situation may look somewhat hopeless:

$$
\begin{aligned}
\sum_{N} \lambda^{N} \mathcal{O}_{[1]}^{N \times N} & =\frac{\lambda(\lambda+1)}{\mu(1-\lambda)^{3}} \\
\sum_{N} \lambda^{N} \mathcal{O}_{[2]}^{N \times N} & =\frac{2 \lambda\left(\lambda^{2}+4 \lambda+1\right)}{\mu^{2}(1-\lambda)^{4}} \\
\sum_{N} \lambda^{N} \mathcal{O}_{[3]}^{N \times N} & =\frac{6 \lambda(\lambda+1)\left(\lambda^{2}+8 \lambda+1\right)}{\mu^{3}(1-\lambda)^{5}} \\
\sum_{N} \lambda^{N} \mathcal{O}_{[4]}^{N \times N} & =\frac{24 \lambda\left(\lambda^{4}+16 \lambda^{2}+36 \lambda^{2}+16 \lambda+1\right)}{\mu^{4}(1-\lambda)^{6}} \\
\sum_{N} \lambda^{N} \mathcal{O}_{[5]}^{N \times N} & =\frac{120 \lambda(\lambda+1)\left(\lambda^{4}+24 \lambda^{3}+76 \lambda^{2}+24 \lambda+1\right)}{\mu^{5}(1-\lambda)^{7}} \\
& \ldots
\end{aligned}
$$

The reason for this is, however, the unjustified restriction to square matrices, see section 2.3 below.

Instead seen from the table is factorization of the averages for the Young diagrams with the single-line tails:

$$
\mathcal{O}_{\left[\Lambda, 1^{k}\right]}=\frac{1}{\mu^{k}} \mathcal{O}_{[\Lambda]} \cdot \prod_{i=0}^{k-1}\left(N^{2}+k+i\right)
$$


This is somewhat similar to the property of extended symmetric group characters $\varphi$ in $[141,142]$. It is a direct counterpart of $(2.31)$ for the complex matrix model: a corollary of the fact that $-\mu$ is the background value of the first time-variable, and everything is invariant under simultaneous shift of $\mu$ and $t_{1}$.

\subsection{Rectangular complex matrix model}

\subsubsection{Partition function and Ward identities}

In fact, there is no need for the matrix $M$ to be square, it can be arbitrary rectangular matrix $N_{1} \times N_{2}$, so that square are the matrices $M M^{\dagger}$ and $M^{\dagger} M$. There is an evident duality between $N_{1}$ and $N_{2}$ in the matrix integral

$$
\mathcal{Z}_{C}\{t\}=\int d^{2} M \exp \left(-\mu \operatorname{Tr} M M^{\dagger}+\sum_{k} t_{k} \operatorname{Tr}\left(M M^{\dagger}\right)^{k}\right)
$$

Considering the deformation

$$
\delta M=\left(M M^{\dagger}\right)^{n} M
$$

of the integration variable in this integral, one deduces that the partition function satisfies the same Virasoro constraints (with $n \geq 0$ ) as in the square case:

$$
(-\mu \frac{\partial}{\partial t_{n+1}}+\sum_{k} k t_{k} \frac{\partial}{\partial t_{k+n}}+\sum_{a=1}^{n-1} \frac{\partial^{2}}{\partial t_{a} \partial t_{n-a}}+\underbrace{\left(N_{1}+N_{2}\right)}_{\alpha} \cdot\left(1-\delta_{n, 0}\right) \frac{\partial}{\partial t_{n}}+\underbrace{N_{1} N_{2}}_{\beta} \cdot \delta_{n, 0}) \mathcal{Z}_{C}\{t\}=0
$$

only the parameters $\alpha$ and $\beta$ are now independent.

\subsubsection{The simplest averages from Virasoro recursion}

From the Virasoro relations one can recursively deduce the Gaussian correlators in the rectangular model:

$$
\begin{aligned}
& \mathcal{O}_{[1]}=\langle\operatorname{Tr} M \bar{M}\rangle=\frac{N_{1} N_{2}}{\mu} \\
& \mathcal{O}_{[2]}=\left\langle\operatorname{Tr}(M \bar{M})^{2}\right\rangle=\frac{N_{1} N_{2}\left(N_{1}+N_{2}\right)}{\mu^{2}} \\
& \mathcal{O}_{[3]}=\left\langle\operatorname{Tr}(M \bar{M})^{3}\right\rangle=\frac{N_{1} N_{2}\left(N_{1}^{2}+3 N_{1} N_{2}+N_{2}^{2}+1\right)}{\mu^{3}} \\
& \mathcal{O}_{[4]}=\frac{N_{1} N_{2}\left(N_{1}+N_{2}\right)\left(N_{1}^{2}+5 N_{1} N_{2}+N_{2}^{2}+5\right)}{\mu^{4}} \\
& \mathcal{O}_{[1,1]}=\left\langle(\operatorname{Tr} M \bar{M})^{2}\right\rangle=\frac{N_{1} N_{2}\left(N_{1} N_{2}+1\right)}{\mu^{2}} \\
& \mathcal{O}_{[2,1]}=\left\langle\operatorname{Tr}(M \bar{M})^{2} \operatorname{Tr} M \bar{M}\right\rangle=\frac{N_{1} N_{2}\left(N_{1}+N_{2}\right)\left(N_{1} N_{2}+2\right)}{\mu^{3}} \\
& \mathcal{O}_{[1,1,1]}=\left\langle(\operatorname{Tr} M \bar{M})^{3}\right\rangle=\frac{N_{1} N_{2}\left(N_{1} N_{2}+1\right)\left(N_{1} N_{2}+2\right)}{\mu^{3}} \\
& \mathcal{O}_{[3,1]}=\frac{N_{1} N_{2}\left(N_{1} N_{2}+3\right)\left(N_{1}^{2}+3 N_{1} N_{2}+N_{2}^{2}+1\right)}{\mu^{4}} \\
& \mathcal{O}_{[2,2]}=\frac{N_{1} N_{2}\left(\left(N_{1}+N_{2}\right)^{2} N_{1} N_{2}+4 N_{1}^{2}+10 N_{1} N_{2}+4 N_{2}^{2}+2\right)}{\mu^{4}} \\
& \mathcal{O}_{[2,1,1]}=\frac{N_{1} N_{2}\left(N_{1}+N_{2}\right)\left(N_{1} N_{2}+2\right)\left(N_{1} N_{2}+3\right)}{\mu^{4}} \\
& \mathcal{O}_{[1,1,1,1]}=\frac{N_{1} N_{2}\left(N_{1} N_{2}+1\right)\left(N_{1} N_{2}+2\right)\left(N_{1} N_{2}+3\right)}{\mu^{4}} \\
& \mathcal{O}_{[5]}=\frac{N_{1} N_{2}\left(N_{1}^{4}+10 N_{1}^{3} N_{2}+20 N_{1}^{2} N_{2}^{2}+10 N_{1} N_{2}^{3}+N_{2}^{4}+15 N_{1}^{2}+40 N_{1} N_{2}+15 N_{2}^{2}+8\right)}{\mu^{5}} \\
& \mathcal{O}_{[4,1]}=\frac{N_{1} N_{2}\left(N_{1}+N_{2}\right)\left(N_{1} N_{2}+4\right)\left(N_{1}^{2}+5 N_{1} N_{2}+N_{2}^{2}+5\right)}{\mu^{5}} \\
& \mathcal{O}_{[3,2]}=\frac{N_{1} N_{2}\left(N_{1}+N_{2}\right)\left(\left(N_{1}^{2}+3 N_{1} N_{2}+N_{2}^{2}\right) N_{1} N_{2}+6 N_{1}^{2}+25 N_{1} N_{2}+6 N_{2}^{2}+18\right)}{\mu^{5}} \\
& \mathcal{O}_{[3,1,1]}=\frac{N_{1} N_{2}\left(N_{1} N_{2}+3\right)\left(N_{1} N_{2}+4\right)\left(N_{1}^{2}+3 N_{1} N_{2}+N_{2}^{2}+1\right)}{\mu^{5}} \\
& \mathcal{O}_{[2,2,1]}=\frac{N_{1} N_{2}\left(N_{1} N_{2}+4\right)\left(\left(N_{1}+N_{2}\right)^{2} N_{1} N_{2}+4 N_{1}^{2}+10 N_{1} N_{2}+4 N_{2}^{2}+2\right)}{\mu^{5}} \\
& \mathcal{O}_{[2,1,1,1]}=\frac{\left(N_{1}+N_{2}\right) N_{1} N_{2}\left(N_{1} N_{2}+2\right)\left(N_{1} N_{2}+3\right)\left(N_{1} N_{2}+4\right)}{\mu^{5}} \\
& \mathcal{O}_{[1,1,1,1,1]}=\frac{N_{1} N_{2}\left(N_{1} N_{2}+1\right)\left(N_{1} N_{2}+2\right)\left(N_{1} N_{2}+3\right)\left(N_{1} N_{2}+4\right)}{\mu^{5}}
\end{aligned}
$$


The FT formula should now include the Fourier transforms in the both variables $N_{1}$ and $N_{2}$

$$
\mathrm{FT}_{\Lambda}=\mu^{|R|} \sum_{N_{1}, N_{2}} \lambda_{1}^{N_{1}} \lambda_{2}^{N_{2}} \mathcal{O}_{\Lambda}^{N_{1} \times N_{2}}
$$

and this immediately provides a simple formula, which substitutes the ugly set (2.45):

$$
\mathrm{FT}_{[m]}=m ! \cdot \frac{\lambda_{1} \lambda_{2}\left(1-\lambda_{1} \lambda_{2}\right)^{m-1}}{\left(1-\lambda_{1}\right)^{m+1}\left(1-\lambda_{2}\right)^{m+1}}
$$

or

$$
\sum_{m} \mathrm{FT}_{[m]} \cdot \frac{z^{m}}{m !}=\frac{\lambda_{1} \lambda_{2}}{1-\lambda_{1} \lambda_{2}} \cdot \frac{1}{\left(1-\lambda_{1}\right)\left(1-\lambda_{2}\right)-z\left(1-\lambda_{1} \lambda_{2}\right)}
$$

The FT functions for other Young diagrams are a little more involved:

$$
\begin{aligned}
& \mathrm{FT}_{[1,1]}=\frac{2 \lambda_{1} \lambda_{2}\left(\lambda_{1} \lambda_{2}+1\right)}{\left(1-\lambda_{1}\right)^{3}\left(1-\lambda_{2}\right)^{3}} \\
& \mathrm{FT}_{[2,1]}=\frac{6 \lambda_{1} \lambda_{2}\left(1-\lambda_{1} \lambda_{2}\right)\left(\lambda_{1} \lambda_{2}+1\right)}{\left.\left(1-\lambda_{1}\right)^{4}\left(1-\lambda_{2}\right)^{4}\right)} \\
& \mathrm{FT}_{[1,1,1]}=\frac{6 \lambda_{1} \lambda_{2}\left(\lambda_{1}^{2} \lambda_{2}^{2}+4 \lambda_{1} \lambda_{2}+1\right)}{\left.\left(1-\lambda_{1}\right)^{4}\left(1-\lambda_{2}\right)^{4}\right)} \\
& \mathrm{FT}_{[3,1]}=\frac{12 \lambda_{1} \lambda_{2}\left(2 \lambda_{1}^{3} \lambda_{2}^{3}-\lambda_{1}^{2} \lambda_{2}^{2}-\lambda_{1}^{2} \lambda_{2}-\lambda_{1} \lambda_{2}^{2}-\lambda_{1} \lambda_{2}+2\right)}{\left(1-\lambda_{1}\right)^{5}\left(1-\lambda_{2}\right)^{5}} \\
& \mathrm{FT}_{[2,2]}=\frac{24 \lambda_{1} \lambda_{2}\left(\lambda_{1}^{3} \lambda_{2}^{3}-2 \lambda_{1}^{2} \lambda_{2}^{2}+\lambda_{1}^{2} \lambda_{2}+\lambda_{1} \lambda_{2}^{2}-2 \lambda_{1} \lambda_{2}+1\right)}{\left(1-\lambda_{1}\right)^{5}\left(1-\lambda_{2}\right)^{5}} \\
& \mathrm{FT}_{[2,1,1]}=\frac{24 \lambda_{1} \lambda_{2}\left(1-\lambda_{1} \lambda_{2}\right)\left(\lambda_{1}^{2} \lambda_{2}^{2}+4 \lambda_{1} \lambda_{2}+1\right)}{\left(1-\lambda_{1}\right)^{5}\left(1-\lambda_{2}\right)^{5}} \\
& \mathrm{FT}_{[1,1,1,1]}=\frac{24 \lambda_{1} \lambda_{2}\left(\lambda_{1}^{3} \lambda_{2}^{3}+10 \lambda_{1}^{2} \lambda_{2}^{2}+\lambda_{1}^{2} \lambda_{2}+\lambda_{1} \lambda_{2}^{2}+10 \lambda_{1} \lambda_{2}+1\right)}{\left(1-\lambda_{1}\right)^{5}\left(1-\lambda_{2}\right)^{5}} \\
& \mathrm{FT}_{[4,1]}=\frac{24 \lambda_{1} \lambda_{2}\left(1-\lambda_{1} \lambda_{2}\right)\left(5 \lambda_{1}^{3} \lambda_{2}^{3}+\lambda_{1}^{2} \lambda_{2}^{2}-6 \lambda_{1}^{2} \lambda_{2}-6 \lambda_{1} \lambda_{2}^{2}+\lambda_{1} \lambda_{2}+5\right)}{\left(1-\lambda_{1}\right)^{6}\left(1-\lambda_{2}\right)^{6}} \\
& \mathrm{FT}_{[3,2]}=\frac{24 \lambda_{1} \lambda_{2}\left(1-\lambda_{1} \lambda_{2}\right)\left(5 \lambda_{1}^{3} \lambda_{2}^{3}-11 \lambda_{1}^{2} \lambda_{2}^{2}+6 \lambda_{1}^{2} \lambda_{2}+6 \lambda_{1} \lambda_{2}^{2}-11 \lambda_{1} \lambda_{2}+5\right)}{\left(1-\lambda_{1}\right)^{6}\left(1-\lambda_{2}\right)^{6}} \\
& \mathrm{FT}_{[3,1,1]}=\frac{24 \lambda_{1} \lambda_{2}\left(5 \lambda_{1}^{4} \lambda_{2}^{4}+16 \lambda_{1}^{3} \lambda_{2}^{3}-6 \lambda_{1}^{3} \lambda_{2}^{2}-6 \lambda_{1}^{2} \lambda_{2}^{3}-18 \lambda_{1}^{2} \lambda_{2}^{2}-6 \lambda_{1}^{2} \lambda_{2}-6 \lambda_{1} \lambda_{2}^{2}+16 \lambda_{1} \lambda_{2}+5\right)}{\left(1-\lambda_{1}\right)^{6}\left(1-\lambda_{2}\right)^{6}} \\
& \mathrm{FT}_{[2,2,1]}=\frac{24 \lambda_{1} \lambda_{2}\left(5 \lambda_{1}^{4} \lambda_{2}^{4}+4 \lambda_{1}^{3} \lambda_{2}^{3}+6 \lambda_{1}^{3} \lambda_{2}^{2}+6 \lambda_{1}^{2} \lambda_{2}^{3}-42 \lambda_{1}^{2} \lambda_{2}^{2}+6 \lambda_{1}^{2} \lambda_{2}+6 \lambda_{1} \lambda_{2}^{2}+4 \lambda_{1} \lambda_{2}+5\right)}{\left(1-\lambda_{1}\right)^{6}\left(1-\lambda_{2}\right)^{6}} \\
& \mathrm{FT}_{[2,1,1,1]}=\frac{24 \lambda_{1} \lambda_{2}\left(1-\lambda_{1} \lambda_{2}\right)\left(5 \lambda_{1}^{3} \lambda_{2}^{3}+49 \lambda_{1}^{2} \lambda_{2}^{2}+6 \lambda_{1}^{2} \lambda_{2}+6 \lambda_{1} \lambda_{2}^{2}+49 \lambda_{1} \lambda_{2}+5\right)}{\left(1-\lambda_{1}\right)^{6}\left(1-\lambda_{2}\right)^{6}} \\
& \mathrm{FT}_{[1,1,1,1,1]}=\frac{120 \lambda_{1} \lambda_{2}\left(\lambda_{1}^{4} \lambda_{2}^{4}+20 \lambda_{1}^{3} \lambda_{2}^{3}+6 \lambda_{1}^{3} \lambda_{2}^{2}+6 \lambda_{1}^{2} \lambda_{2}^{3}+54 \lambda_{1}^{2} \lambda_{2}^{2}+6 \lambda_{1}^{2} \lambda_{2}+6 \lambda_{1} \lambda_{2}^{2}+20 \lambda_{1} \lambda_{2}+1\right)}{\left(1-\lambda_{1}\right)^{6}\left(1-\lambda_{2}\right)^{6}}
\end{aligned}
$$


However, they satisfy elegant sum rules, the analogue of (2.26) and (2.27):

$$
\begin{aligned}
\mathrm{FT}_{[1]} & =\frac{\lambda_{1}}{\left(1-\lambda_{1}\right)^{2}} \cdot \frac{\lambda_{2}}{\left(1-\lambda_{2}\right)^{2}}, \\
\mathrm{FT}_{[2]}+\mathrm{FT}_{[1,1]} & =\frac{2 \lambda_{1}}{\left(1-\lambda_{1}\right)^{3}} \cdot \frac{2 \lambda_{2}}{\left(1-\lambda_{2}\right)^{3}}, \\
2 \cdot \mathrm{FT}_{[3]}+3 \cdot \mathrm{FT}_{[2,1]}+\mathrm{FT}_{[1,1,1]} & =\frac{6 \lambda_{1}}{\left(1-\lambda_{1}\right)^{4}} \cdot \frac{6 \lambda_{2}}{\left(1-\lambda_{2}\right)^{4}}, \\
6 \cdot \mathrm{FT}_{[4]}+8 \cdot \mathrm{FT}_{[3,1]}+3 \cdot \mathrm{FT}_{[2,2]}+6 \cdot \mathrm{FT}_{[2,1,1]}+\mathrm{FT}_{[1,1,1,1]} & =\frac{24 \lambda_{1}}{\left(1-\lambda_{1}\right)^{5}} \cdot \frac{24 \lambda_{2}}{\left(1-\lambda_{2}\right)^{5},} \\
24 \cdot \mathrm{FT}_{[5]}+30 \cdot \mathrm{FT}_{[4,1]}+20 \cdot \mathrm{FT}_{[3,2]}+20 \cdot \mathrm{FT}_{[3,1,1]}+15 \cdot \mathrm{FT}_{[2,2,1]} & +10 \cdot \mathrm{FT}_{[2,1,1,1]}+\mathrm{FT}_{[1,1,1,1,1]} \\
& =\frac{120 \lambda_{1}}{\left(1-\lambda_{1}\right)^{6}} \cdot \frac{120 \lambda_{2}}{\left(1-\lambda_{2}\right)^{6},}
\end{aligned}
$$

As in the case of Hermitian matrix model, one easily recognizes in the coefficients here the appropriately normalized symmetric group characters $\varphi_{R}(\Lambda)$ from $[141,142]$. Hence, one immediately obtains the general formula

$$
\sum_{\Lambda \vdash k} \varphi_{[k]}(\Lambda) \cdot \mathrm{FT}_{\Lambda}=\frac{k ! \lambda_{1}}{\left(1-\lambda_{1}\right)^{k+1}} \cdot \frac{k ! \lambda_{2}}{\left(1-\lambda_{2}\right)^{k+1}}
$$

and, for $R=[k]$,

$$
\left.\frac{1}{d_{R}} \chi_{R}\left\{\frac{1}{n} \frac{\partial}{\partial t_{n}}\right\} \log \mathcal{Z}_{C}\right|_{t=0}=\sum_{\Lambda \vdash k} \varphi_{[k]}(\Lambda) \cdot \mathcal{O}_{[\Lambda]}^{N_{1} \times N_{2}}=\frac{1}{\mu^{k}} \frac{\Gamma\left(N_{1}+k\right)}{\Gamma\left(N_{1}\right)} \frac{\Gamma\left(N_{2}+k\right)}{\Gamma\left(N_{2}\right)}
$$

Moreover, the factorization persists for an arbitrary $R$ and it is especially simple for the single-hook diagrams $R=\left[k, 1^{l-1}\right]$ :

$$
\sum_{\Lambda \vdash|R|} \varphi_{[R]}(\Lambda) \cdot \mathrm{FT}_{[\Lambda]}=\sum_{\Lambda \vdash|R|} \varphi_{[R]}(\Lambda) \cdot\left(\sum_{N_{1}, N_{2}} \lambda_{1}^{N_{1}} \lambda_{2}^{N_{2}} \cdot \mathcal{O}_{[\Lambda]}^{N_{1} \times N_{2}}\right)=\frac{|R| ! \cdot \lambda_{1}^{l}}{\left(1-\lambda_{1}\right)^{|R|+1}} \cdot \frac{|R| ! \cdot \lambda_{2}^{l}}{\left(1-\lambda_{2}\right)^{|R|+1}}
$$

where $l$ is the number of lines in $R$. For more complicated diagrams $R$, there are simple factors in the numerator, e.g. for $R=[3,2]$ the factorial 5 ! gets substituted by $4 ! \cdot(3 \lambda+2)$, while the transposition to $R=[2,2,1]$ changes it for $4 ! \cdot(3+2 \lambda)$. Similarly, for $R=[2,2]$ the factorial 4 ! changes for $3 ! \cdot(2 \lambda+2)$. We discuss the origins and implications of these formulas elsewhere.

The complete perturbative solution to the rectangular complex model, i.e. an explicit formula for arbitrary Gaussian correlator is now provided by a somewhat simpler counterpart of (2.30):

$$
\mathcal{O}_{\Lambda}=\frac{1}{\mu^{|\Lambda|}} \sum_{R \vdash|\Lambda|} \frac{D_{R}\left(N_{1}\right) D_{R}\left(N_{2}\right)}{d_{R}} \cdot \psi_{R}(\Lambda)
$$


and similarly for the partition function

$$
\mathcal{Z}_{C}\{t\}=\sum_{R} \frac{1}{\mu^{|R|}} \frac{D_{R}\left(N_{1}\right) D_{R}\left(N_{2}\right)}{d_{R}} \cdot \chi_{R}\{t\}
$$

One can immediately associate this partition function with a partition function from the family (2.38):

$$
\mathcal{Z}_{C}\{t\}=Z_{(1,2)}\left\{\mu, N_{1}, N_{2} \mid t_{k}\right\}=Z_{(2,2)}\left\{\mu, N \mid \bar{t}_{k}=\delta_{k, 1}, t_{k}\right\}
$$

its complex matrix model representation found in [140] being slightly different from $\mathcal{Z}_{C}\{t\}$.

The factorization property (2.46) also survives, with a simple modification:

$$
\mathcal{O}_{\left[\Lambda, 1^{k}\right]}=\frac{1}{\mu^{k}} \mathcal{O}_{[\Lambda]} \cdot \prod_{i=0}^{k-1}\left(N_{1} N_{2}+k+i\right)
$$

A relative complexity of the FT formulas for the averages (2.53) can be attributed to dependence of the factor on the product of two $N_{1} N_{2}$, which can be modeled by action of the Casimir-type operator $\lambda_{1} \lambda_{2} \frac{\partial^{2}}{\partial \lambda_{1} \partial \lambda_{2}}$ on $F T_{[\Lambda]}$ and can not be reduced in any way to just a shift of variables (what could be achieved by an adequate integral transform if it was the action of just $\lambda \frac{\partial}{\partial \lambda}$ ).

For $N_{2}=1$, i.e. for the vector model with $N_{1}=N$, this factorization extends to all Gaussian correlators:

$$
\mathcal{O}_{[\Lambda]}^{N \times 1}=\frac{1}{\mu^{|\Lambda|}} \prod_{i=0}^{|\Lambda|-1}(N+i)=\frac{\Gamma(N+|\Lambda|)}{\mu^{|\Lambda|} \Gamma(N)}
$$

in particular, the generating function (2.51) of single-traced averages in the case of vector model reduces to

$$
\sum_{N=0}^{\infty} \mathcal{O}_{[m]}^{N \times 1} \cdot \lambda^{N}=\sum_{N=0}^{\infty} \frac{(N+m-1) !}{(N-1) !} \cdot \lambda^{N}=\sum_{N=0}^{\infty} \frac{\Gamma(N+m)}{\Gamma(N)} \cdot \lambda^{N}=m ! \cdot \frac{\lambda}{(1-\lambda)^{m+1}}
$$

Moreover, the operators and thus their averages for all other Young diagrams $\Lambda=\left\{m_{1} \geq\right.$ $\left.m_{2} \geq \ldots \geq 0\right\}$ depend only on their sizes $|\Lambda|=\sum_{k} m_{k}$, thus the above answer for the single-line diagrams $\Lambda=[|\Lambda|]=\left[\sum_{k} m_{k}\right]$ is exhaustive in this case:

$$
\mathcal{O}_{[\Lambda]}^{N \times 1}=\left\langle\prod_{k}\left(\sum_{i=1}^{N} M_{i} \bar{M}_{i}\right)^{m_{k}}\right\rangle=\mu^{N} \prod_{i=1}^{N} \int d M_{i} d \bar{M}_{i} e^{-\mu M_{i} \bar{M}_{i}}\left(\sum_{i=1}^{N} M_{i} \bar{M}_{i}\right)^{|\Lambda|}=\mathcal{O}_{[|\Lambda|]}^{N \times 1}
$$

\subsubsection{W-representation}

The $W$-representation for the rectangular complex model can be read off from formulas of [140] upon its identification with $Z_{(1,2)}$ :

$$
\mathcal{Z}_{C}\{t\}=\exp \left\{\frac{1}{\mu}\left(N_{1} N_{2} t_{1}+\left(N_{1}+N_{2}\right) \hat{L}_{1}+\hat{W}_{1}\right)\right\} \cdot 1
$$


with

$$
\begin{aligned}
\hat{L}_{1} & =\sum_{m}(m+1) t_{m+1} \frac{\partial}{\partial t_{m}}, \\
\hat{W}_{1} & =\sum_{a, b} a b t_{a} t_{b} \frac{\partial}{\partial t_{a+b-1}}+(a+b+1) t_{a+b+1} \frac{\partial^{2}}{\partial t_{a} \partial t_{b}}
\end{aligned}
$$

In variance with (2.18)-(2.19), when the $W$-operator has the grading +2 , these operators have the grading +1 , which is related with the fact that the bare action is given by the shift of the first time, i.e. $-\mu$ is the background value of $t_{1}$, while, in the Gaussian Hermitian case, it is $t_{2}$ whose background value is equal to $-\mu / 2$.

\section{On the universal structure of Virasoro-like constraints}

In fact, in many different models the construction of Ward identities follows one and the same line. The principal player in the game is the special set of operators originating from those in the bare action. We call the non-bilinear operators in the bare action keystone, and the set of interest is built from them by various kinds of contractions leading to tree operators and loop operators. These are the only ones needed for the RG-completion of the theory, and they do not necessarily include all possible operators allowed by symmetries. Instead, these are exactly the operators emerging in the derivation of Ward identities along the lines of $[6-10]$.

\subsection{Keystone operators and their RG-descendants}

Usually in theoretical physics, one begins from the study of QFT models at some intermediate energy scale, and describes them as a collection of certain degrees of freedom (say, moving (quasi)particles, or spins at fixed positions, etc), which can interact with each other. Accordingly, we write down an action consisting of kinetic terms which are quadratic in fields, and certain interaction, which, within the context of the present paper, we call non-quadratic keystone operators. In the case of Hermitian matrix model, this starting action is

$$
-\frac{\mu}{2} \operatorname{Tr} M^{2}+\operatorname{Tr} M^{3}
$$

The main feature of QFT is that in general such an action turns out to be drastically changed by quantum corrections modulo a few notable exceptions, which include the fundamental theory of nature, the Standard Model of elementary particles, and the starting action gets "dressed" and acquires an absolutely different form. New interaction terms are immediately generated, and the resulting action has many operators with the entire variety of couplings. In the Hermitian matrix model, this corresponds to switching from (3.1) to

$$
-\frac{\mu}{2} \operatorname{Tr} M^{2}+\sum_{k=1}^{\infty} t_{k} \operatorname{Tr} M^{k}
$$

Usually this dressing process is described in terms of the renormalization group (RG) flows in the moduli space of couplings (time-variables) $\left\{t_{k}\right\}$, and the resulting action is the one 
which is $R G$-complete: no more operators are needed to describe any correlator that is nonvanishing. One of the basic problems in QFT is to find the RG-completion of the given starting action, i.e. to identify all the RG-descendants of the given keystone operators. The thing is that this set can actually be smaller than all the operators which are allowed by symmetries, this phenomenon is well known in the conventional QFT as the existence of UV- or IR-renormalizable models. There, however, one usually deals with theories that possess the space-time, where one can additionally distinguish between local and nonlocal operators, and often only local operators are included into the RG considerations, at least, in the UV region. The standard renormalizability in the UV region is then usually restricted by various types of unitarity constraints and requires the RG-completion by local operators. In matrix models as well as in general in string theory, there is no space time, locality does not play any special role and unitarity is present by the construction. Criteria for the RG-completeness are instead related to existence of rich Ward identities, known in matrix models under the name of Virasoro/W-constrains (because these are the algebras to which they belong, as Borel subalgebras, in the simplest matrix models). In general QFT, these Ward identities underlying the theory of RG flows are representations of the peculiar algebra of rooted trees, the corresponding construction is known as BogoliubovZimmermann theory and we are going to briefly review it in this section. Since our main task in this paper is lifting the matrix model theory to the tensor models, we rely upon the BZ-formalism in the presentation of [100] (see also [173] for an interesting related issue). Similar considerations can be also found in [83, 84, 174, 175].

Though we do not go that far in this paper, the first really interesting tensor model to analyze within this context is the rainbow model of [25] (see also [77, 78] for earlier works). In the rainbow model, each index of the rank- $r$ tensor field belongs to the representation of its own unitary group and, as a consequence, all the $r+1$ fields merging at the hypertetrahedron (simplex) vertex are different. In result, there are $r+1$ different propagators, each being a tube/cable with $r$ lines of different coloring, and the total number of different colorings is $\frac{r(r+1)}{2}$. For the simplest non-trivial case of $r$ this is 6 , hence, the name "rainbow". The keystone operators are provided by the tetrahedron vertices (since the vertex is tetrahedron in the first non-trivial (tensor) case of $r=3$, for the sake of simplicity, we always call it just tetrahedron), which can be depicted as follows:

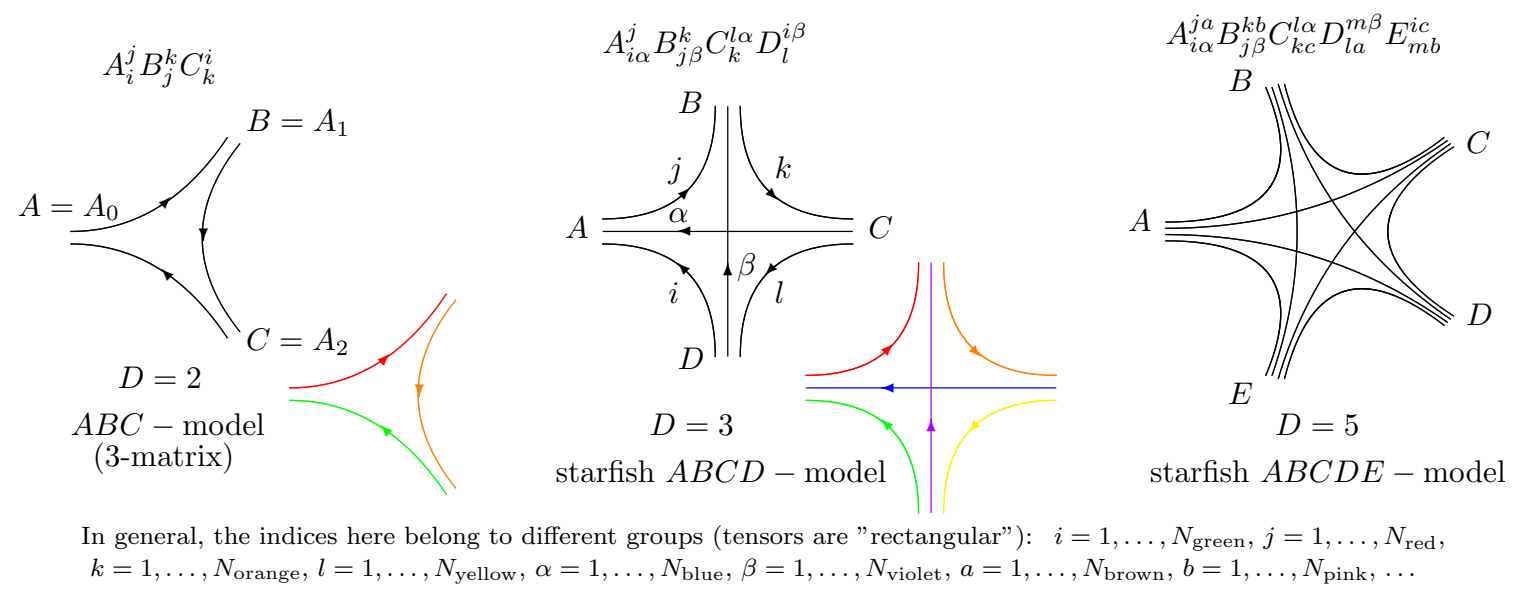


The first task in the study of this model is to build the RG-descendants of these keystone operators and describe this emerging set in some efficient way. The first step on this way i.e. in constructing the tree and loop operators from the keystones is, in fact, universal, while the relation of the loop and tree operators is model-dependent and its investigation is still a piece of art. In this section, we describe the universal part of the story, while in the following two we use much simpler tensor models to illustrate a possibility of artistic steps. Lifting these considerations to the rainbow models themselves remains for the future.

As to the Ward identities, they can be formulated at two different levels. The easy and universal step to be actually described below is constructing recursion relations between particular Gaussian averages, which can allow one to build them one after another. Usually this recursion is just in the power (the number) of fields in the operator. A more artistic step is to collect these recursions into equations in terms of generating functions. As we saw in section 2, this can be actually done in different ways, useful for different purposes. What is important, at the level of generating functions, one can actually move away from the Gaussian point and consider other phases. Once equations for the generating functions are known, this non-perturbative treatment is provided just by a shift of the time-variables $t \longrightarrow T+t$. As soon as such a description of the rainbow models is worked out (not in the present paper, yet), one is able to treat the tetrahedron vertices non-perturbatively, as lifting of the theory of Dijkgraaf-Vafa phases from the matrix to rainbow tensor models.

\subsection{Tree operators as the base of RG-complete set}

We now remind the first steps of the RG-completion of the given keystone interaction. They are absolutely universal and applicable to any QFT model. We will be illustrating this general construction by two examples, relevant for the purpose of this paper: the rectangular complex and rainbow $A B C D$ models.

1) Specify integration variables (fields) and the kinetic term (Gaussian weight), e.g.

$$
\int d^{2} M e^{-\mu \operatorname{Tr} M M^{\dagger}}, \quad \text { or } \quad \int d^{2} A d^{2} B d^{2} C d^{2} D e^{-\mu \operatorname{Tr} A \bar{A}+\operatorname{Tr} B \bar{B}+\operatorname{Tr} C \bar{C}+\operatorname{Tr} D \bar{D}}
$$

2) Select a keystone operator or a pair of these, e.g.

$$
\mathcal{K}=\operatorname{Tr}\left(M M^{\dagger}\right)^{2}
$$

or, in the square-matrix case,

$$
\left(\mathcal{K}=\operatorname{Tr} M^{4}\right) \oplus\left(\mathcal{K}=\operatorname{Tr} \bar{M}^{4}\right)
$$

for the first matrix model in (3.3), and, for the second model, in (3.3),

$$
(\mathcal{K}=[A B C D]) \oplus(\overline{\mathcal{K}}=[\bar{D} \bar{C} \bar{B} \bar{A}])
$$

which we depict as fat points (black $\oplus$ white) with four (in these examples) thick ( $r$-fat) external lines. The tin lines will be used to describe the internal structure of propagators 
and vertices: in matrix models, the thick lines are called fat and made from a pair of think lines. For rank- $r$ tensors, the thick lines are tubes/cables containing $r$ thin lines, which, for the rainbow models, are all of different colors. Moreover, the cables can contain different (but not arbitrary) combinations of $r$ colors and thus are themselves multicolored. The fat points, where different cables merge, can have a complicated internal structure describing reshuffling of the thin lines between the cables, and they can be very different. To handle this variety, we agree to denote by the thick points only the keystone vertices, while all other types of cable mergers will be induced from them, actually, by the Feynman diagrams.

Indeed, the thick points and lines are the ones describing vertices and propagators in the ordinary Feynman diagrams for the keystone interaction. In fact, these Feynman diagrams generate new operators. In conventional QFT, we do not pay too much attention to this, because these new operators are usually non-local, and only some of them contain essentially local contributions (like, say, the tadpoles or the UV-divergent diagrams). However, in theories where one does not care about the space-time and locality, like in the case of matrix models, all operators arising from the Feynman diagrams are relevant.

3) Construct new connected operators from these by connecting some of the thick lines, i.e. by applying the operations

$$
\operatorname{Tr} \frac{\partial}{\partial M^{\dagger}} \otimes \frac{\partial}{\partial M} \quad \text { or } \quad \operatorname{Tr} \frac{\partial}{\partial \bar{A}} \otimes \frac{\partial}{\partial A}+\operatorname{Tr} \frac{\partial}{\partial \bar{B}} \otimes \frac{\partial}{\partial B}+\operatorname{Tr} \frac{\partial}{\partial \bar{C}} \otimes \frac{\partial}{\partial C}+\operatorname{Tr} \frac{\partial}{\partial \bar{D}} \otimes \frac{\partial}{\partial D}
$$

Let us consider the second keystone operator (3.5). If applied once to a pair of points, the operation (3.7) provides an operator with six external legs $\operatorname{Tr} M^{3} \bar{M}^{3}$. In our notations of section 2.1.3, this is depicted as

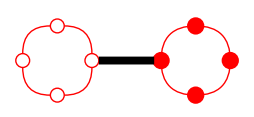

$$
\operatorname{Tr} M^{3} \bar{M}^{3}
$$

However, for illustrative purposes, in this paragraph we temporarily return to the standard Feynman graph notation, though it will be used to enumerate the local operators. Then, the Feynman diagrams with six ( $\operatorname{Tr} M^{3} \bar{M}^{3}$ drawn above) and eight external legs (if the operation is applied twice to a set of six points) look like

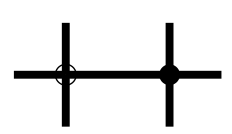

$\operatorname{Tr} M^{3} \bar{M}^{3}$

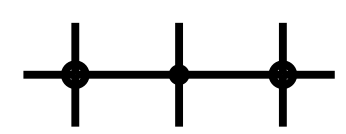

$\operatorname{Tr} M^{3} \bar{M} M^{3} \bar{M}$

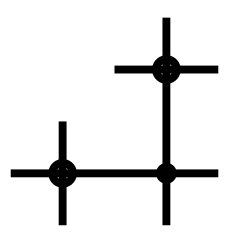

$\operatorname{Tr} M^{6} \bar{M}^{2}$

and so on. In this picture, we show an example of the square matrix model, for the rectangular case there are no chiral operators for the role of keystone ones, only $\mathcal{K}=\operatorname{Tr}\left(M M^{\dagger}\right)^{2}$, 
thus, all vertices will be the same (no black and white), and the emerging operators will be just two instead of three: $\operatorname{Tr}\left(M M^{\dagger}\right)^{3}$ and $\operatorname{Tr}\left(M M^{\dagger}\right)^{4}$, according to the higher symmetry of the model. For the rainbow model, the pictures will remain the same, but the internal structure of emerging operators (contraction of indices) will be a little more involved, and can be easily depicted in terms of the thin-line diagrams. The total number of emerging operators will not actually increase too much, because the growth of the number of fields ( $A, B, C, D$ instead of a single $M$ ) will be compensated by the increased symmetry: the $r$-colorings of propagator tubes/cables will not be arbitrary and there will be at most four options per each thick line, with additional constraints that all the thick lines in each tetrahedron vertex are different.

One can also apply the same operation twice to just two points, giving rise to operators with four external legs:

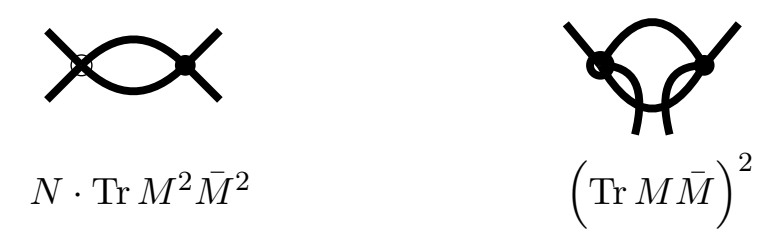

but these will be "loop" rather than "tree" operators. If we look at these operators in the thin-line representation, then, for the matrix models, it gets clear that all tree operators are just the ordinary single-trace operators, while the loop operators are either single- or multi-trace operators:

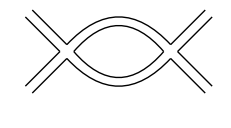

$$
N \times \operatorname{Tr} M^{2} \bar{M}^{2}
$$

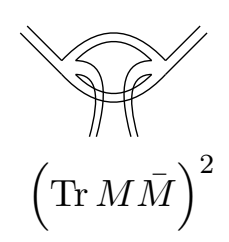

However, in the tensor case, there is no better term than tree and loop operators for the substitutes of the matrix model single- and multi-traces.

Note that, in the case of matrix models, all planar diagrams actually give rise to the single-trace operators (times traces of unity, which are just powers of $N$ ), while the true multi-trace operators emerge only from the non-planar diagrams. The number of traces is related to the degree of non-planarity (that is, to the genus of the surface obtained by putting all external lines together in a cyclic order). The counterpart of this feature for the tensor models depends on the choice of keystone operators and also plays a role in structure of the extended partition functions and the Ward identities.

4) Define the extended partition function by putting all tree operators in the action:

$$
\mathcal{Z}(t)=d^{2} \mathcal{M} \exp \left(-[\mathcal{M} \overline{\mathcal{M}}]+t \mathcal{K}+\bar{t} \overline{\mathcal{K}}+\sum_{\text {trees }} t_{\text {tree }} \mathcal{K}_{\text {tree }}\right)
$$

$\mathcal{M}$ is a symbolic unifying notation for the dynamical field. The keystone operators can be considered as associated with the simplest tree consisting of one vertex (black or white, in the chiral case). 
5) Virasoro like constraints reflect the invariance of extended partition function under the changes of integration variable, generated by a gradient of any operator in the action,

$$
\delta_{\text {tree }} \mathcal{M}=\frac{\partial}{\partial \overline{\mathcal{M}}} \mathcal{K}_{\text {tree }}
$$

(the exact correspondence between the indices in $\mathcal{M}$ and $\overline{\mathcal{M}}$ is dictated by the kinetic term). In other words, as any Ward identities, they are essentially averages of the equations of motion. This transformation changes any term in the extended action by

$$
t_{\text {tree }^{\prime}} \frac{\partial}{\partial \bar{M}} \mathcal{K}_{\text {tree }} \frac{\partial}{\partial \bar{M}} \mathcal{K}_{\text {tree }^{\prime}}
$$

which is by definition again a tree operator, this produces a term like

$$
\sum_{T^{\prime}} t_{T}^{\prime} \frac{\partial}{\partial t_{T \circ T^{\prime}}} \mathcal{Z}
$$

in the Virasoro constraints with a clear notion of tree composition $T \circ T^{\prime}$ (the tree $T$ is attached by some of its vertices to the tree $T^{\prime}$ at some of its vertices, and all possible choices are summed up).

Actually the trees are rooted and it is also convenient to consider variation, where $\mathcal{M}$ gradient is taken w.r.t. the fields in the root vertex only, then the composition operation o of the rooted trees becomes even simpler: one attaches a root of one tree to any vertex of another.

6) However, the Jacobian of the transformation (3.9) contains loop operators, i.e. is no longer expanded in the trees. Moreover, there is no reason for it to be expressed via any number of derivatives w.r.t. the tree time-variables $t_{\text {tree }}$, as it happens in the matrix models. In other words, the extended partition function (3.8) looks to be not RG-complete.

From this point, we have two obvious ways to proceed: introduce more terms into the extended action to make it RG complete or to look for a factorization of loop operator averages at large $N$ and to get a closed set of constraints, at least, in this limit (i.e. to construct a counterpart of the spectral curve with the hope to build further a counterpart of the AMM/EO topological recursion over it).

\subsection{The simplest recursions}

If we begin with the action $-\mu[\mathcal{M} \overline{\mathcal{M}}]+t_{\circ} \mathcal{K}+t_{\bullet} \overline{\mathcal{K}}$, with $\mathcal{K}$ being of the forth power in $\mathcal{M}$, and consider its variation $\delta \overline{\mathcal{M}}=d_{M} \mathcal{K}$, then we get

$$
-\mu\left[M d_{M} \mathcal{K}\right]+t_{\bullet} d_{\bar{M}} \overline{\mathcal{K}} d_{M} \mathcal{K}=-4 \mu \mathcal{K}+16 t_{\bullet} \mathcal{K}_{1, \overline{1}}
$$

or, pictorially (with combinatorial factors omitted),

$$
\left(-\mu_{0}+t_{\bullet} \longrightarrow+\bigcirc\right) \cdot: e^{t_{\bullet} \circ} \cdot e^{t_{0}}:: \cong 0
$$


The last term comes from the Jacobian $d_{M} \delta \bar{M}=d_{M} d_{M} \mathcal{K}$. The Ward identity says that the Gaussian average of this sum should vanish (the fact that vanishing takes place only after averaging is expressed by the sign $\cong$ instead of equality). The Gaussian averages of the two chiral operators in the sum are zero, but one should take into account the contributions proportional to $t_{\bullet}$ and coming from the exponentials. This is similar to the usual story: in

$$
-\mu \frac{\partial}{\partial t_{n}}+\sum_{k} k t_{k} \frac{\partial}{\partial t_{k+n}}
$$

one should either put $t$ to zero and stay with just

$$
-\mu \frac{\partial}{\partial t_{n}}
$$

or first differentiate over $t_{k}$ to stay with

$$
-\mu \frac{\partial^{2}}{\partial t_{k} \partial t_{n}}+k \frac{\partial}{\partial t_{k+n}}
$$

In result, we get in the first non-vanishing order in $t$

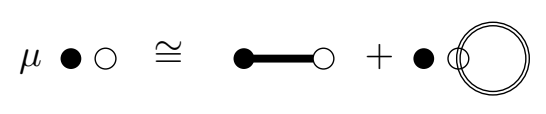

In application to the particular model, one should also insert combinatorial factors and put the normal ordering around the operator $\bullet$. Taking all this into account, together with vanishing of the Gaussian averages of chiral operators, which explains the elimination of disconnected averages, one recognizes a trivial identity. Not quite trivial is only the matching of combinatorics at all orders in $\mu^{-1}$.

Similarly one can draw a generic tree Virasoro constraint, with one tree attached to all vertices of another, in the above example each of the two trees consists of a single vertex.

\subsection{BZ exponential and rooted trees}

As already mentioned in [100] and [25], one of the ways to construct generating functions of trees is provided by the Bogoliubov-Zimmermann forest formula

$$
e^{\hat{V}}=1+\sum_{\text {forests } \mathcal{F}} \frac{1}{\operatorname{Tree}(\mathcal{F}) !} \prod_{\text {trees }} \frac{\hat{V}_{\mathcal{T}}}{\sigma_{\mathcal{F}} \mathcal{T} !}
$$

for the expansion of the exponentiated vector field

$$
\hat{V}=\sum_{\alpha=1}^{P} v^{\alpha} \partial_{\alpha}
$$


Notation in this expansion is best explained pictorially:

$$
\begin{aligned}
& e^{\hat{V}}=\sum_{n=0}^{\infty} \frac{\hat{V}^{n}}{n !}=1+v^{\alpha} \partial_{\alpha}+\frac{1}{2} v^{\gamma} \partial_{\gamma} v^{\alpha} \partial_{\alpha}+\frac{1}{6} v^{\gamma} \partial_{\gamma} v^{\beta} \partial_{\beta} v^{\alpha} \partial_{\alpha}+\ldots= \\
& 0 j_{0}^{\infty} \sum_{0}^{\infty} \\
& =1+\left(v^{\alpha}+\frac{1}{2} v^{\gamma}\left(\partial_{\gamma} v^{\alpha}\right)+\frac{1}{6} v^{\gamma}\left(\partial_{\gamma} v^{\beta}\right)\left(\partial_{\beta} v^{\alpha}\right)+\frac{1}{6} v^{\beta} v^{\gamma}\left(\partial_{\beta} \partial_{\gamma} v^{\alpha}\right)+\ldots\right) \partial_{\alpha}+ \\
& \$ 8 \\
& \text { is } \\
& i_{0}^{\infty} \\
& \sum_{0}^{\infty} \\
& +\frac{1}{2} \cdot \overbrace{\left(v^{\alpha}+\frac{1}{2} v^{\gamma}\left(\partial_{\gamma} v^{\alpha}\right)+\ldots\right)\left(v^{\beta}+\frac{1}{2} v^{\gamma}\left(\partial_{\gamma} v^{\beta}\right)+\ldots\right) \partial_{\alpha} \partial_{\beta}}^{v^{\alpha} v^{\beta} \partial_{\alpha} \partial_{\beta}+\frac{1}{2} v^{\gamma}\left(\partial_{\gamma} v^{\alpha}\right) v^{\beta} \partial_{\alpha} \partial_{\beta}+\frac{1}{2} v^{\alpha} v^{\gamma}\left(\partial_{\gamma} v^{\beta}\right) \partial_{\alpha} \partial_{\beta}+\frac{1}{4} v^{\gamma}\left(\partial_{\gamma} v^{\alpha}\right) v^{\gamma^{\prime}}\left(\partial_{\gamma^{\prime}} v^{\alpha}\right) \partial_{\alpha} \partial_{\beta}+\ldots}+ \\
& \text { ¿๐ं } \\
& \begin{array}{l}
+\frac{1}{6} \overbrace{\left(v^{\alpha}+\ldots\right)\left(v^{\beta}+\ldots\right)\left(v^{\gamma}+\ldots\right) \partial_{\alpha} \partial_{\beta} \partial_{\gamma}}^{v^{\alpha} v^{\beta} v^{\gamma} \partial_{\alpha} \partial_{\beta} \partial_{\gamma}+\ldots}+\ldots= \\
=1+\sum_{\mathcal{F}} \frac{1}{\operatorname{Tree}(\mathcal{F}) !} \prod_{\mathcal{T} \in \mathcal{F}} \frac{\hat{V}_{\mathcal{T}}}{\sigma_{\mathcal{T}} \mathcal{T} !}=: \exp \left(\sum_{\mathcal{T}} \frac{\hat{V}_{\mathcal{T}}}{\sigma_{\mathcal{T}} \mathcal{T} !}\right):
\end{array}
\end{aligned}
$$

In other words, at any vertex (except for the root) we put a vector field $\hat{V}$, which acts on $v$ at exactly the next vertex towards the root. The emerging combinatorial factors in the sums are of two kinds: the trivial ones, associated with the forests (inverse factorials of the number of trees $\operatorname{Tree}(\mathcal{F})$ in the forest), i.e. coefficients of the Maclaurin expansion of the exponential, and the less trivial ones, associated with the trees: they are described by recursively defined Connes-Moscovici factorials [176]

$$
\mathcal{T} !=\operatorname{Vert}(\mathcal{T}) \cdot \prod_{\tau} \mathcal{T}_{\tau} !
$$

where $\operatorname{Vert}(\mathcal{T})$ is the number of vertices in $\mathcal{T}$, while the product goes over all sub-trees $\tau \subset \mathcal{T}$, in which $\mathcal{T}$ decays if the root (the bottom arrow) is cut away. $\sigma_{\mathcal{T}}$ is just the tree 
symmetry factor (in the above pictures, it is different from unity only for the last tree in the first line, in this case, it is equal to 2). The (non-trivial) fact that the forest dependence of combinatorial factors is so simple allows one to rewrite the exponential of the vector field $\hat{V}$ as a normal ordered exponential of another vector field (while one could expect that it would be some non-trivial poly-vector). The normal ordering means that all the differential operators are put to the right of all the coefficient functions,

$$
: e^{\hat{V}}:=\sum_{n=0}^{\infty}: v^{\alpha_{1}} \partial_{\alpha_{1}} v^{\alpha_{2}} \partial_{\alpha_{2}} \ldots v^{\alpha_{n}} \partial_{\alpha_{n}}: \equiv \sum_{n=0}^{\infty} v^{\alpha_{1}} v^{\alpha_{2}} \ldots v^{\alpha_{n}} \partial_{\alpha_{1}} \partial_{\alpha_{2}} \ldots \partial_{\alpha_{n}}
$$

Then this new vector field is just the sum over all trees in the box in the second line of (3.15): this is the statement of the last equation in this formula.

\subsection{The Bogoliubov-Zimmermann tensor model}

The sum in (3.13) goes over the rooted graphs with vertices of arbitrary valence. In particular tensor models, one needs to restrict it to a particular valence, say $p+1$, or, more precisely, $(p, 1)$. Each such vertex has one exiting link and $p$ incoming ones, and contributes a factor of $v^{\otimes p}\left(\partial^{\otimes p} v\right)$. Thus, contributions with a given $p$ will be the only surviving ones, if $v$ are polynomials of exactly power $p$, then the answer for each tree will consist of the product of $v(x)$ 's at the end-points of the graph times an $x$-independent number obtained by contraction of indices at all vertices. Any vertex of higher valence will automatically drop out, the vertices with lower valence will contain extra powers of $x$ and can be eliminated by putting $x=0$ in the expression for the graph with amputated external vertices.

In other words, if the vector field $\hat{V}$ should be a (rank- $p$ tensor-valued) vector field,

$$
\hat{V}\{x\}=v_{I_{1} \ldots I_{p}}^{J} x^{I_{1}} \ldots x^{I_{p}} \frac{\partial}{\partial x^{J}}=\frac{\partial v(x, \bar{x})}{\partial \bar{x}_{J}} \frac{\partial}{\partial x^{J}}
$$

with $\bar{x}$-linear $v(x, \bar{x})=v_{I_{1} \ldots I_{p}}^{J} x^{I_{1}} \ldots x^{I_{p}} \bar{x}_{J}$, and one considers its exponential, $e^{\hat{V}}$ applied to some function of $x$, say, $e^{x}$, then

$$
\mathcal{Z}\{x\}=e^{\mathcal{F}\{x\}}=e^{\hat{V}\{x\}} e^{x}
$$

will be expanded in graphs with valences $r+1$ or less. It looks very much like a $W$ representation of the tree (quasiclassical) approximation to a peculiar tensor model

$$
Z_{\mathrm{BZ}}=\int d \mathcal{M} d \overline{\mathcal{M}} \exp (-\mu \sum_{I} \mathcal{M}^{I} \overline{\mathcal{M}}_{I}+t \underbrace{\sum_{I_{1}, \ldots, I_{p}, J} v_{I_{1} \ldots I_{r}}^{J} \mathcal{M}^{I_{1}} \ldots \mathcal{M}^{I_{p}} \overline{\mathcal{M}}_{J}}_{v^{J}(\mathcal{M}) \overline{\mathcal{M}}_{J}})
$$

which we naturally name $B Z$ model.

In the context of the usual tensor models like the rainbow one, the indices $I$ and $J$ play the role of multi-indices, labeling the multicolored tubes/cables and the coefficients $v$ encode their coupling via the keystone (e.g. tetrahedron) vertex, in the rainbow model occasionally $p=r$. Of course, all the indices can have different multi-coloring with one being distinguished, associated with the contravariant index $J$, this is similar to the colored (red) 
partition function in (4.2). The extended partition function contains all the tree operators, which, in this case, all contain a single field $\mathcal{M}$ and arbitrarily large number of $\mathcal{M}$ :

$$
\mathcal{Z}_{\mathrm{BZ}}\left\{t_{\text {trees }}\right\}=\int d \mathcal{M} d \overline{\mathcal{M}} \exp (-\mu \cdot \sum_{I} \mathcal{M}^{I} \overline{\mathcal{M}}_{I}+\sum_{\text {trees }} t_{\mathcal{T}} \cdot \underbrace{T_{\mathcal{T}}(\mathcal{M}, \overline{\mathcal{M}})}_{\left[\mathcal{M}_{\mathcal{T}}^{\otimes \nu}(\mathcal{T}), \overline{\mathcal{M}}\right]_{\mathcal{T}}})
$$

Here $T_{\mathcal{T}}(\mathcal{M}, \overline{\mathcal{M}})=\left[\mathcal{M}^{\otimes \nu(\mathcal{T})}, \overline{\mathcal{M}}\right]_{\mathcal{T}}=T_{\mathcal{T}}^{I}(\mathcal{M}) \cdot \overline{\mathcal{M}}_{I}$ is a polynomial in $\mathcal{M}$ of degree $\nu_{\mathcal{T}}=$ $1+(p-1) \cdot \operatorname{Vert}\left(\mathcal{T}\right.$ and a linear function in $\overline{\mathcal{M}}$ with the coefficients from $v^{\otimes \operatorname{Vert}(\mathcal{T})}$ and the conversion of indices dictated by the tree $\mathcal{T}$.

Each $\overline{\mathcal{M}}$-linear function placed inside the Gaussian integral acts on the $\mathcal{M}$-dependent objects as a vector field, $V^{J}(\mathcal{M}) \overline{\mathcal{M}}_{J} \cong \hat{V} \equiv v^{J}(\mathcal{M}) \frac{\partial}{\partial \mathcal{M}^{J}}$, this is basically the meaning in which (3.15) and (3.20) are the same (up to the factors $t$ and $\mu$ ). In (3.21) the vector field is a sum over all possible rooted trees, taken with arbitrary coupling constants (timevariables) $t_{\mathcal{T}}$.

Composition of the vector fields induces an associative non-commutative algebra structure on the "group algebra" of the rooted trees, with multiplication

$$
\hat{T}_{\mathcal{T}_{1}} \circ \hat{T}_{\mathcal{T}_{2}}=\left(T_{1}^{I}(\mathcal{M}) \frac{\partial T_{2}^{J}(\mathcal{M})}{\partial \mathcal{M}^{I}}\right) \frac{\partial}{\partial \mathcal{M}_{J}}=T_{1 \circ 2}(\mathcal{M})^{J} \frac{\partial}{\partial \mathcal{M}_{J}}=\hat{T}_{\mathcal{T}_{1} \circ \mathcal{T}_{2}}
$$

which looks like just attaching a tree $\mathcal{T}_{1}$ to a vertex of $\mathcal{T}_{2}$, summed over all vertices. Of course, when the valence is restricted by $p$, the attachment is possible only to the vertices which have free valencies. This operation will play a crucial role in the structure of the universal Ward identities in section 3.6.

\subsection{Archetypical/universal Virasoro constraint}

The Ward identities in the BZ model form an archetypical set of the Virasoro constraints, which is then inherited this or that way by all other QFT models.

There are two kinds of transformations generated by the "white" functions (i.e. with no free/external indices): $\overline{\mathcal{M}}$-independent $Q(\mathcal{M})$ and $\overline{\mathcal{M}}$-linear $S(\mathcal{M}, \overline{\mathcal{M}})=S^{J}(\mathcal{M}) \overline{\mathcal{M}}_{\mathcal{J}}$. The two kinds of identities reflect the invariance with respect to the shifts

$$
\delta \overline{\mathcal{M}}_{I}=\frac{\partial Q}{\partial \mathcal{M}^{I}} \longrightarrow \mu\left\langle\langle\underbrace{\mathcal{M}^{I} \frac{\partial Q}{\partial \mathcal{M}^{I}}}_{\operatorname{deg}_{Q} \cdot Q(\mathcal{M})}\rangle\right\rangle=\sum_{\mathcal{T}} t_{\mathcal{T}} \cdot\left\langle\langle\underbrace{\frac{\partial T_{\mathcal{T}}}{\partial \overline{\mathcal{M}}_{I}} \frac{\partial Q}{\partial \mathcal{M}^{I}}}_{\hat{T}_{\mathcal{T}} Q(\mathcal{M})}\rangle\right\rangle
$$

and

$$
\delta \mathcal{M}^{I}=\frac{\partial S}{\partial \overline{\mathcal{M}}_{I}} \longrightarrow \mu\left\langle\langle\underbrace{\overline{\mathcal{M}}_{I} \frac{\partial S}{\partial \overline{\mathcal{M}}^{I}}}_{\hat{S}}\rangle\right\rangle=\sum_{\mathcal{T}} t_{\mathcal{T}} \cdot\left\langle\langle\underbrace{\frac{\partial S}{\partial \overline{\mathcal{M}}^{I}} \frac{\partial T_{\mathcal{T}}}{\partial \mathcal{M}_{I}}}_{\hat{S} \hat{T}_{\mathcal{T}}}\rangle\right\rangle+\left\langle\left\langle\frac{\partial^{2} S}{\partial \overline{\mathcal{M}}_{I} \partial \mathcal{M}^{I}}\right\rangle\right\rangle
$$

The averages are in the model (3.21), i.e. not Gaussian; moreover, they should be understood as taken in the background $\mathcal{M}$-field (or with an additional insertion of the source term like $\left.\exp \left(\mathcal{J}^{I} \overline{\mathcal{M}}_{I}\right)\right)$, otherwise all averages are just zero. 
An essential difference between (3.23) and (3.24) is that the former does not contain a Jacobian contribution, while the latter does. If we restrict to these two types of transformations, at least the one in (3.24), then the $t$-linear terms contain just the compositions of trees, i.e. are trees again, and can be expressed as derivatives w.r.t. the variables $t_{T \circ Q}$ and $t_{S \circ T}$. Jacobian contribution in (3.24) can not, but instead it can be treated within the context of (3.23).

\subsection{Relation to Feynman diagrams}

At vanishing $t$, the Ward identities (3.23) and (3.24) and their $t$-derivatives provide concrete recursion relations between particular Gaussian correlators. We can instead calculate these Gaussian correlators directly by the Wick theorem. Comparing these two types of calculations, one can note that the Ward identities contain only action of the vector field $\hat{S}$ on the vector field $\hat{T}_{\mathcal{T}}$, while the Wick theorem calculation also contains contributions with $\hat{T}_{\mathcal{T}}$ acting on $\hat{S}$.

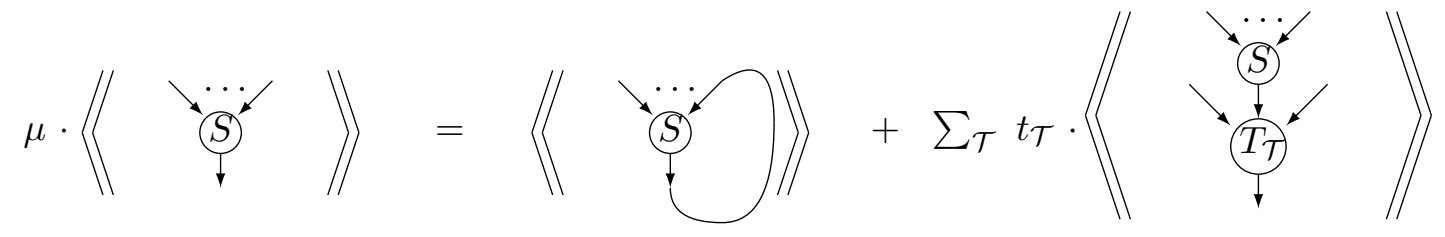

The resolution of this "paradox" is that the Wick theorem application actually provides a combination of two Ward identities.

\subsection{BZ resolvents}

Of course, expansions like (3.13) exist for all other functions of vector fields, not obligatory exponentials, the only difference is in combinatorial coefficients. The tree dependent coefficient is provided by a recursive analogue of the Connes-Moscovici (recursive Maclaurin) formula (3.16). In particular, one can define a BZ-resolvent as a Laplace transform of the BZ exponential (3.13),

$$
\frac{1}{z-\hat{V}}=\int_{0}^{\infty} e^{-z t} e^{z \hat{V}}
$$

\subsection{The message}

Generalization from the vector to exponentials of poly-vector fields, i.e. to actions nonlinear in $\overline{\mathcal{M}}$, do not possess any enhanced reparametrization symmetry: closed algebra is formed only for the scalar and vector transforms. Indeed, the three types of terms in the Virasoro constraints have $\overline{\mathcal{M}}$-powers

$$
\bar{n} \oplus(\bar{n}+\bar{k}-1) \oplus(\bar{n}-1)
$$

and potentially closed are only the two cases: either $\bar{k}=1$ or $\bar{n}=1$. A possible way out is to make an infinite tower of powers $\bar{k}$, tying them to the powers of $\mathcal{M}$, as it is done in the complex matrix model, where the operators $\operatorname{Tr}\left(M M^{\dagger}\right)^{k}$ have $\bar{k}=k$. This, however, leads to problems with the Jacobians. 
A hope can be that the loop operators arising from the Jacobians are algebraically dependent on the tree operators, like it happens in the one-matrix models. This can happen in particular models, specifically, in the rainbow model. However, this option deserves further investigation.

An alternative remark is that the action of trees is defined on all poly-vectors, and the Jacobians are needed only from the acting tree, thus they are always the same. In case of poly-vectors, this does not provide the necessary recursion (because no poly-vectors arise in this way from the vector fields), still this provides a set of relations describing the extended poly-vector generating functions as representations of the tree algebra.

What definitely exists are recursions like (5.2) between the Gaussian correlators, which allow one to evaluate all averages recursively in the power of fields: first all correlators with two fields, then with four, then with six, and so on. These recursions are obtained from the generic Ward identities when they are expanded in powers of time-variables around the Gaussian point. Such evaluation of the Gaussian correlators is the necessary stage of development in the theory of tensor models, which can hardly be avoided, and we presented some examples in this paper. Lifting to the true Ward identities is important for non-perturbative calculations, i.e. for the study of expansions around non-Gaussian points, and for development of related more sophisticated techniques: character expansions, integrability (KP/Toda and Hurwitz), quasiclassical integrability, spectral curves, AMM/EO topological recursion, $W$ and Kontsevich representations etc. This is also a long work for the future.

\section{RG-closed tensor generalization of the complex matrix model}

\subsection{Partition function}

Substitute now the rectangular matrix $M_{i}^{j}$ by a tensor $A_{i}^{j_{1}, \ldots, j_{r-1}}$ of rank $r$ with one covariant and $r-1$ contravariant indices. Adding a conjugate tensor $\bar{A}_{j_{1}, \ldots, j_{r-1}}^{i}$, one can make a kinetic term and consider the following model:

$$
Z_{T C}=\int d^{2} A \exp \left(\sum_{i, j_{1}, \ldots, j_{r-1}} A_{i}^{j_{1} \ldots j_{r-1}} \bar{A}_{j_{1} \ldots j_{r-1}}^{i}\right)
$$

where the measure is induced by the norm $\|\delta A\|^{2}=\delta A_{i}^{j_{1} \ldots j_{r-1}} \delta \bar{A}_{j_{1} \ldots j_{r-1}}^{i}$. Each index can be rotated by its own unitary group of its own size, so that the model has the symmetry $\otimes_{a=1}^{r} \mathrm{U}\left(N_{a}\right)$. In fact, the symmetry in (4.1) is much higher: the integral is just the same as for the rectangular matrix model of size $N_{1} \times\left(N_{2} \cdot \ldots \cdot N_{r}\right)$, with the symmetry $\mathrm{U}\left(N_{1}\right) \otimes \mathrm{U}\left(N_{2} \cdot \ldots \cdot N_{r}\right)$. What distinguishes the tensor model from such an enveloping matrix model is the choice of allowed operators. If they have lower (tensor-model) symmetry than that of the matrix model, their correlators are not among the matrix model ones and should be calculated from the dedicated Ward identities, which need to be separately derived. For this, it is important to know the what we call "RG (renormalization-group)-closed" sets of operators, for which the Ward identities are self-sufficient and self-consistent, at least, in the certain large- $N$ limits. In this section, we provide some primary examples of such 
considerations. For the sake of simplicity, we draw the pictures and write the formulas for the model with $r=3$, which we call RGB (red-green-blue) or Aristotelian (since Aristotle distinguished 3 colors in the rainbow [177], [178, p. 107], only Newton raised the number to the canonical seven). In most cases, generalization to arbitrary $r$ is obvious just like the further resolution of colors in the spectrum.

\subsection{Notation: two types of diagrams}

If one thinks about the tensor models, the main problem is to find a workable description of indices and their contractions. Algebraically, there are no notions like matrix product and trace and even a small number of tensors can be contracted in many different ways. Drawing pictures can help, but this interferes with already existing technique of the Feynman diagrams in QFT. In fact, this problem already exists with matrices, but there a simple way out was invented: the Feynman propagators in Yang-Mills theory are depicted as double lines, and gauge invariance requires the thin lines to be trivially rearranged (cyclically connected) at the vertices. For rank $r$ tensor fields, the Feynman propagators are thick lines, tubes or cables consisting of $r$ thin lines. The real problem are interaction vertices, where these thin lines can be interconnected in many complicated ways. Thus, there is a separate task of drawing the vertices, i.e. of drawing the gauge-invariant local operators, and most of pictures in this and other tensor model papers are trying to depict them. Things are greatly simplified in the rainbow models of [25], where thin lines have as many different colorings as only possible, and this both decreases the number of invariant operators and simplifies pictures for them.

Coming back to the simplest possible Gaussian rainbow model (4.1), we use it to introduce the convenient notation, which allow one to separately treat the drawings (diagrams) for the local operators and for the Feynman diagrams for their correlators and interactions.

The vertices ("local" operators) are represented by "thin" diagrams, where the vertices are fields (tensors) and they connected by thin colored lines, which describe the contraction of indices.

The Feynman diagrams ("thick diagrams") describe averages (correlators of "local" operators): they are also diagrams where "local" operators shrink to thick points of different kinds (with different internal structures) and different external valencies associated with the fields, which were the vertices in the "thin" diagrams. The Feynman propagators are depicted as thick ( $r$-colored) lines (tubes/cables).

This double-level diagram technique, where the Feynman vertices and propagators have their own non-trivial internal structure, is getting more and more important in modern theory: for example, something very similar appears under the name of double-fat diagrams in the effective theory of arborescent knots in [179-181].

Kinetic term and Feynman propagator. As an operator, $\operatorname{Tr} A \bar{A}$ in (4.1) can be depicted by three thin lines of different colors, connecting two vertices $A$ and $\bar{A}$, which we will usually depict as a circle of "unit length". Directions of arrows depend on the choice of covariant and contravariant indices, which is not essential for the models in this paper. However, we choose them in accord with the tetrahedron model, despite it is beyond the 
scope of the present text.

$$
\begin{aligned}
& \operatorname{Tr} A \bar{A} \quad=A_{i_{r}}^{j_{g} \alpha_{b}} \bar{A}_{j_{g} \alpha_{b}}^{i_{r}}=A_{i_{r}}^{j_{g} \alpha_{b}} \bar{A}_{j_{g} \alpha_{b}}^{i_{r}}=A \rightleftarrows \bar{A}=\square=\square=\bullet \\
& \langle A \bar{A}\rangle=A \longrightarrow \bar{A}=\delta_{i}^{i^{\prime}} \cdot \delta_{j^{\prime}}^{j} \cdot \delta_{\alpha^{\prime}}^{\alpha} \\
& \langle\operatorname{Tr} A \bar{A}\rangle=\square=\square=\square=N_{r} \cdot N_{b} \cdot N_{g}
\end{aligned}
$$

From the point of view of Feynman diagrams, this operator is just a vertex of valence 2 , to be denoted by a fat point with just two vacancies for possible attachment of the Feynman propagators. The propagator depicted by the thick black line is itself defined by the same kinetic term $\operatorname{Tr} A \bar{A}$ or, if one prefers, as a correlator of $A$ and $\bar{A}$. In this sense, the thick black line is a tube or a cable consisting of three thing colored lines. The average $\langle\operatorname{Tr} A \bar{A}\rangle$ is a closed circle made from the Feynman propagator.

"Single-trace" non-chiral operators. The matrix model single-trace operators $\operatorname{Tr}\left(A A^{\dagger}\right)^{k}=\operatorname{Tr}(A \bar{A})^{k}$ are now substituted by $K_{k}=K_{k}$ and $\tilde{K}_{k}=K_{k}$
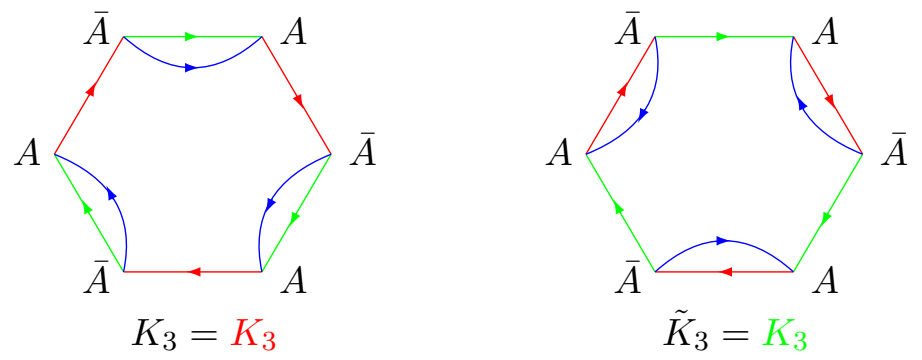

which we often call respectively red and green circles (or benzene rings) of length $k$ referring to the color and number of single-line sides. The circle of unit length can be considered as either "red" or "green", $\mathcal{K}_{1}=\mathcal{K}_{1}$. In fact, there are also a blue cousins $\mathcal{K}_{m}$ of the green operators, but we begin from just red, then add green, and blue then automatically emerges.

\subsection{Ordinary Virasoro constraints for an oversimplified tensor model}

The simplest possibility for an extension of the partition function (4.1) is to include only the operators $K_{k}$, i.e. only the red circles, this makes one of the colors distinguished:

$$
\mathcal{Z}\{t\}=\int d^{2} A \exp \left(-\mu \operatorname{Tr} A \bar{A}+\sum_{k} t_{k} K_{k}\right)
$$

and we call this "red" tensor model. Considering a deformation

$$
\delta A=\frac{1}{n+1} \frac{\partial K_{n+1}}{\partial \bar{A}}=\frac{1}{n+1} \nabla\left(K_{n+1}\right)
$$

of the integration variable in this integral, we deduce that $\mathcal{Z}$ satisfies the nearly conventional Virasoro constraints (with $n \geq 0$ ):

$$
(-\mu \frac{\partial}{\partial t_{n+1}}+\sum_{k} k t_{k} \frac{\partial}{\partial t_{k+n}}+\sum_{a=1}^{n-1} \frac{\partial^{2}}{\partial t_{a} \partial t_{n-a}}+\underbrace{\left(N_{r}+N_{g} N_{b}\right)}_{\alpha}\left(1-\delta_{n, 0}\right) \frac{\partial}{\partial t_{n}}+\underbrace{N_{r} N_{g} N_{b}}_{\beta} \cdot \delta_{n, 0}) Z\{t\}=0
$$


or

for the resolvent

$$
\left(-\mu+\frac{\alpha}{z}\right) \rho+\rho^{2}+\frac{\beta}{z^{2}}+\nabla_{z} \rho=0
$$

$$
\rho(z)=\nabla_{z} \log Z=\frac{1}{Z} \sum_{n=1}^{\infty} \frac{1}{z^{n+1}} \frac{\partial}{\partial t_{n}} \log Z
$$

at $t=0$.

The Virasoro constrains (4.4) provide a rigorous identification of the minimallyextended partition function (4.2) with that of rectangular matrix model: they differ only in interpretation of the parameters $\alpha$ and $\beta$. As usual, we consider this identification for the partition functions analytically continued in $N$.

\subsection{Spectral curve as the leading term of the genus expansion}

Neglecting $\nabla_{z} \rho$, we get the spectral curve

$$
\begin{aligned}
\rho_{0} & =\frac{1}{2}\left(\mu-\frac{\alpha}{z}-\sqrt{\left(\mu-\frac{\alpha}{z}\right)^{2}-\frac{4 \beta}{z^{2}}}\right)= \\
& =\frac{\beta}{\mu z^{2}}+\frac{\alpha \beta}{\mu^{2} z^{3}}+\frac{\left(\alpha^{2}+\beta\right) \beta}{\mu^{3} z^{4}}+\frac{\left(\alpha^{2}+3 \beta\right) \alpha \beta}{\mu^{4} z^{5}}+\frac{\left(\alpha^{4}+6 \alpha^{2} \beta+2 \beta^{2}\right) \beta}{\mu^{5} z^{6}}+\ldots
\end{aligned}
$$

or

$$
y^{2}=\left(\mu-\frac{\alpha}{z}\right)^{2}-\frac{4 \beta}{z^{2}}
$$

which describes the single-trace averages in the limit of large $\alpha$ and $\beta$.

\subsection{Examples of averages}

The simplest correlators are recursively deduced from (4.4), and they are basically the same as in section 2.3.2, if expressed through $\alpha$ and $\beta$ :

$$
\begin{aligned}
& \mathcal{O}_{[1]}=\left\langle\mathcal{K}_{1}\right\rangle=\underline{\underline{\mu}} \\
& \mathcal{O}_{[2]}=\left\langle\mathcal{K}_{2}\right\rangle=\frac{\overline{\alpha \beta}}{\mu^{2}} \\
& \mathcal{O}_{[3]}=\left\langle\mathcal{K}_{3}\right\rangle=\frac{\frac{\beta\left(\alpha^{2}+\beta+1\right)}{\mu^{3}}}{\mathcal{O}^{3}} \\
& \mathcal{O}_{[4]}=\frac{\alpha \beta\left(\frac{\alpha^{2}+3 \beta+5}{\mu^{4}}\right)}{\mu^{4}} \\
& \mathcal{O}_{[5]}=\frac{\beta\left(\frac{\alpha^{4}+6 \alpha^{2} \beta+2 \beta^{2}}{\mu^{5}}+15 \alpha^{2}+10 \beta+8\right)}{\mu^{5}} \\
& \mathcal{O}_{[1,1]}=\left\langle\mathcal{K}_{1} \mathcal{K}_{1}\right\rangle=\frac{\beta(\beta+1)}{\mu^{2}} \\
& \mathcal{O}_{[2,1]}=\left\langle\mathcal{K}_{2} \mathcal{K}_{1}\right\rangle=\frac{\alpha \beta(\beta+2)}{\mu^{3}} \quad \mathcal{O}_{[1,1,1]}=\left\langle\mathcal{K}_{1} \mathcal{K}_{1} \mathcal{K}_{1}\right\rangle=\frac{\beta(\beta+1)(\beta+2)}{\mu^{3}} \\
& \mathcal{O}_{[3,1]}=\frac{\beta(\beta+3)\left(\alpha^{2}+\beta+1\right)}{\mu^{4}} \quad \mathcal{O}_{[2,2]}=\frac{\beta\left(\alpha^{2} \beta+4 \alpha^{2}+2 \beta+2\right)}{\mu^{4}} \\
& \mathcal{O}_{[2,1,1]}=\frac{\alpha \beta(\beta+2)(\beta+3)}{\mu^{4}} \quad \mathcal{O}_{[1,1,1,1]}=\frac{\beta(\beta+1)(\beta+2)(\beta+3)}{\mu^{4}} \\
& \mathcal{O}_{[4,1]}=\frac{\alpha \beta(\beta+4)\left(\alpha^{2}+3 \beta+5\right)}{\mu^{5}} \quad \mathcal{O}_{[3,2]}=\frac{\alpha \beta\left(\alpha^{2} \beta+6 \alpha^{2}+\beta^{2}+13 \beta+18\right)}{\mu^{5}} \\
& \mathcal{O}_{[3,1,1]}=\frac{\left(\alpha^{2}+\beta+1\right) \beta(\beta+3)(\beta+4)}{\mu^{5}} \mathcal{O}_{[2,2,1]}=\frac{\beta(\beta+4)\left(\alpha^{2} \beta+4 \alpha^{2}+2 \beta+2\right)}{\mu^{5}} \\
& \mathcal{O}_{[2,1,1,1]}=\frac{\alpha \beta(\beta+2)(\beta+3)(\beta+4)}{\mu^{5}} \quad \mathcal{O}_{[1,1,1,1,1]}=\frac{\beta(\beta+1)(\beta+2)(\beta+3)(\beta+4)}{\mu^{5}} \\
& \mathcal{O}_{[6]}=\frac{\alpha \beta\left(\frac{\alpha^{4}+10 \alpha^{2} \beta+10 \beta^{2}}{\mu^{6}}+35 \alpha^{2}+70 \beta+84\right)}{\varkappa^{6}}
\end{aligned}
$$

As usual, every step of recursion produces an extra power of $\mu^{-1}$. The underlined terms in the single-trace averages (for the single-line Young diagrams in the first column) are described by the spectral curve formula (4.7). From these formulas, it is clear what the genus zero approximation actually means in this case: one picks up the highest possible powers in $\alpha$ and $\beta$, irrespective of actual relation between $\alpha$ and $\beta$. Of course, other interesting large- $N$ limits are also possible in this case. 
These correlators satisfy an analogue of the sum rules (2.56), e.g.

$$
\begin{array}{r}
\mathcal{O}_{[2]}+\mathcal{O}_{[1,1]}=\frac{(\alpha+\beta+1) \beta}{\mu^{2}}=\frac{N_{r}\left(N_{r}+1\right) N_{g} N_{b}\left(N_{g} N_{b}+1\right)}{\mu^{2}} \\
\left.\frac{1}{d_{[k]} \mathcal{Z}} \chi_{[k]}\left\{\frac{\partial}{\partial t_{n}}\right\} \mathcal{Z}\right|_{t=0}=\sum_{\Lambda \vdash k} \varphi_{[k]}(\Lambda) \cdot \mathcal{O}_{[\Lambda]}^{N_{1} \times N_{2}}=\frac{\Gamma\left(N_{r}+k\right)}{\Gamma\left(N_{r}\right)} \frac{\Gamma\left(N_{g} N_{b}+k\right)}{\Gamma\left(N_{g} N_{b}\right)}
\end{array}
$$

and so on, but they are not expressible through the FT functions (which are now triplegraded) as simply as their matrix model predecessors in section 2.3.2. Moreover, one can obtain formulas similar to those in s.2.3 for arbitrary correlators and the partition function

$$
\begin{aligned}
\mathcal{O}_{\Lambda} & =\frac{1}{\mu^{|\Lambda|}} \sum_{R \vdash|\Lambda|} \frac{D_{R}\left(N_{r}\right) D_{R}\left(N_{g} N_{b}\right)}{d_{R}} \cdot \psi_{R}(\Lambda) \\
Z\{t\} & =\sum_{R} \frac{1}{\mu^{|R|}} \frac{D_{R}\left(N_{r}\right) D_{R}\left(N_{g} N_{b}\right)}{d_{R}} \cdot \chi_{R}\{t\}
\end{aligned}
$$

in terms of sizes of tensors, or with the replace $\left(N_{r}, N_{g} N_{b}\right) \rightarrow \alpha / 2 \pm \sqrt{\alpha^{2} / 4-\beta}$ in terms of $\alpha$ and $\beta$.

Factorization (2.31) in the case, when the Young diagram has a single-line tale, is now:

$$
\mathcal{O}_{\left[\Lambda, 1^{n}\right]}=\left.\mu^{\beta}\left(\frac{\partial}{\partial t_{1}}\right)^{n}\left(\frac{1}{\mu^{\beta}} \mathcal{O}_{\Lambda}\right)\right|_{t=0}=\mu^{\beta}\left(-\frac{\partial}{\partial \mu}\right)^{n}\left(\frac{1}{\mu^{\beta}} \mathcal{O}_{\Lambda}\right)=\frac{1}{\mu^{n}} \mathcal{O}_{\Lambda} \cdot \prod_{i=0}^{n-1}(\beta+|\Lambda|+i)
$$

where $\mathcal{O}_{\Lambda} \sim \mu^{-|\Lambda|}$, so that

$$
\mathcal{O}_{\left[\Lambda, 1^{n}\right]}=\frac{1}{\mu^{n}} \mathcal{O}_{[\Lambda]} \prod_{i=|\Lambda|}^{|\Lambda|+n-1}(\beta+i)
$$

for arbitrary Young diagram $\Lambda$. This property resembles a similar structure of symmetric group characters, see $[141,142]$. Irreducible in the above table are just the averages in the first column and the averages in boxes.

\subsection{Recursions}

With the above formulas, it is easy to check the simplest recursions following from the Virasoro constraints (4.4) and their $t$-derivatives at $t=0$. In fact, they can also be considered as examples of the basic Ward identity

$$
\mu\left\langle A \frac{\partial \mathcal{K}}{\partial A}\right\rangle=\left\langle\frac{\partial^{2} \mathcal{K}}{\partial A \partial \bar{A}}\right\rangle
$$

reflecting the invariance under the shift $\delta \bar{A}=\frac{\partial \mathcal{K}}{\partial A}$ of the integration variable. Here $\mathcal{K}$ can be any product of the operators $\mathcal{K}_{m}$, and, for homogeneous $\mathcal{K}$, the 1.h.s. is proportional to $\mathcal{K}$ itself. Indices are suppressed: their contraction is obvious at the both sides. The r.h.s. contains contributions from gluing (underlined) and cutting, cutting of the circle of unit length provides a factor of $\alpha$, gluing the two vertices of $\mathcal{K}_{1}$ (if it is present at the 1.h.s.) provides a factor of $\beta$ (double underlined): 


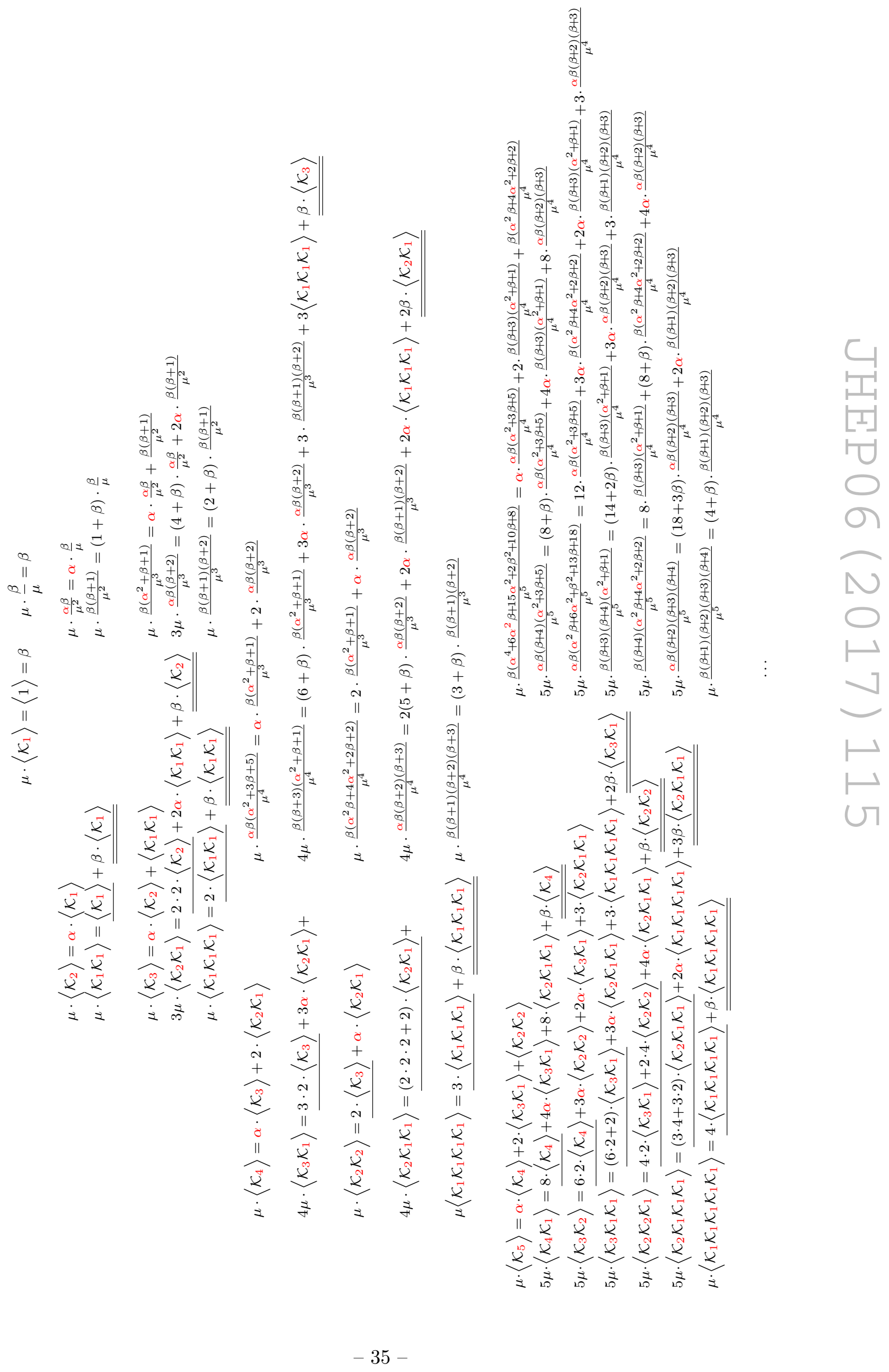


Such corollaries of the universal recursion (4.15) is the kind of relations that one can first look for in more complicated tensor models, where their generating functions like (4.4) are not immediately available.

\subsection{Integrability properties: does Virasoro imply integrability?}

Since the model (4.2) is equivalent to the rectangular complex matrix model, which, according to $[114,170-172]$ is integrable, at least, when the matrix is square, the partition function $Z\{t\}$ at $\beta=\alpha^{2} / 4$ is actually again a $\tau$-function of the (forced) Toda chain hierarchy, however, it is a different solution to the hierarchy: $C_{k}$ in the determinant representation (2.37) is now of the form

$$
C_{k}=\int_{0}^{\infty} d x x^{k} \exp \left(-\mu x+\sum_{k} t_{k} x^{k}\right)
$$

In fact, since the model (4.2) has the representation (4.12), similarly to the rectangular complex model (2.59), it can be associated [163] for arbitrary $\alpha$ and $\beta$ with $Z_{(1,2)}$

$$
Z\{t\}=Z_{(1,2)}\left\{\mu, N_{r}, N_{g} N_{b} \mid t_{k}\right\}
$$

which is a hypergeometric KP $\tau$-function [140, 158-161]. Moreover, by switching on another set of times, one makes of it $Z_{(2,2)}$ which is a Toda lattice $\tau$-function, however, different from the Toda chain.

\subsubsection{W-representation}

The $W$-representation for this model also can be read off from formulas of [140] upon its identification with $Z_{(1,2)}$ :

$$
Z\{t\}=\exp \left\{\frac{1}{\mu}\left(\beta t_{1}+\alpha \hat{L}_{1}\{t\}+\hat{W}_{1}\{t\}\right)\right\} \cdot 1
$$

with

$$
\begin{aligned}
\hat{L}_{1}\{t\} & =\sum_{m}(m+1) t_{m+1} \frac{\partial}{\partial t_{m}} \\
\hat{W}_{1}\{t\} & =\sum_{a, b} a b t_{a} t_{b} \frac{\partial}{\partial t_{a+b-1}}+(a+b+1) t_{a+b+1} \frac{\partial^{2}}{\partial t_{a} \partial t_{b}}
\end{aligned}
$$

\subsection{The message}

Thus, the "red" tensor model (4.2) is not really tensor: it is equivalent to a rectangular matrix model, all its averages are deducible from a single set of Virasoro constraints and satisfy the linear sum rules, i.e. the model is solvable in extreme sense.

The only thing we lose are the advantages of the Fourier transform in $N$. It worked perfectly for the single-trace correlators $\mathcal{O}_{[k]}$ in the Hermitian model, i.e. in the "vertical" direction in the table of averages. However, in the "horizontal" direction, a "composite" variable $N_{1} N_{2}$ appears through the factorization formula, and there is no nice way to express the Fourier transform in such variable through the two separate transformations in 
$N_{1}$ and $N_{2}$ (unless both $N$ 's are large and the Fourier sums can be substituted by integrals). Still, the double Fourier transform allowed one to find simple combinations of averages, where the complexities drop out, and this led to the powerful sum rules. At the tensor level, however, we have a triple (for three colorings) Fourier transform and the triply-composed variable $N_{r} N_{g} N_{b}$, with no efficient way to work with it. Of course, the correlators in the red model can still be found, but with the reference to the matrix models, not by an adequate triple-Fourier technique. Thus, if one wants to look at tensor peculiarities in the simplest possible place, the issue of the Fourier transform is the right choice. In the next section, we see the need for a solution to this problem even better.

Still, to really penetrate in the field of tensor models and see the deviations from the matrix model intuition, we need more than just the "red" model. Thus, we add blue to red, and then green emerges as promised in [177]. Actually, we begin from adding green.

\section{A non-trivial RG-closed extension of the Aristotelian tensor model}

\subsection{The red-green partition function}

Of course, in considerations of the previous section one could use operators $\mathcal{K}_{k}$ instead of $\mathcal{K}_{k}$. The only difference between them is the switch of some contravariant indices to covariant ones, and it plays no role at this stage. The Gaussian averages of $\mathcal{K}_{k}$ are given by the same formulas (4.9).

However, we can also consider mixed correlators like $\left\langle\mathcal{K}_{k} \mathcal{K}_{m}\right\rangle$, i.e. study a less trivial "mixed" partition function

$$
Z\{t, t\}=\int d^{2} A \exp \left(-\mu \operatorname{Tr} A \bar{A}+\sum_{k} t_{k} K_{k}+\sum_{k} t_{k} K_{k}\right)
$$

For this purpose the standard Virasoro constraints are no longer sufficient. Moreover, to get the Ward identities, we now need to deform, say, $K_{k}$ by $\nabla\left(K_{m}\right)$, and this produces new operators like

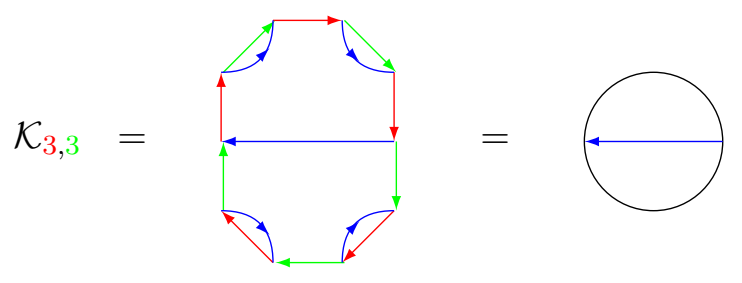

This means that (5.1) is not RG-complete, and more operators and time-variables should be added to its extended action. Clearly, just $\mathcal{K}_{k, m}$ are not enough: further variations of these operators can produce even more. Typical examples of diagrams contributing to the 
full set of Ward identities are

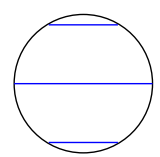

$k t \frac{\partial}{\partial t}$

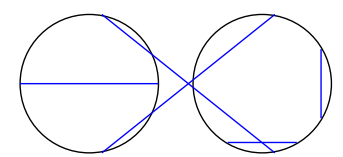

$\frac{\partial^{2}}{\partial t \partial t}$

The question is what the minimal RG-completion is.

We can look for it by examining the tree and loop descendants of the keystone operators, but a more practical approach is to do this directly at the Ward identities. Namely, there is a universal recursion for the Gaussian averages,

$$
\mu\left\langle A_{i_{r} \alpha_{b}}^{j_{g}} \frac{\partial \mathcal{K}}{\partial A_{i_{r} \alpha_{b}}^{j_{g}}}\right\rangle=\left\langle\frac{\partial^{2} \mathcal{K}}{\partial A_{i_{r} \alpha_{b}}^{j_{g}} \partial \bar{A}_{j_{g}}^{i_{r} \alpha_{b}}}\right\rangle
$$

applicable to arbitrary operator $\mathcal{K}$. It can be considered either as the Ward identity for the shift $\delta \bar{A}=\partial \mathcal{K} / \partial A$ evaluated at the point where all $t_{k}=0$, or just as a simple consequence of the Wick theorem (Wick recursion, result of just one propagator insertion). The point is that the operators arising at the r.h.s. can be of more general nature than those at the 1.h.s. That is, if one takes for $\mathcal{K}$ arbitrary functions of keystone operator(s), their $A, \bar{A}$ derivatives contain both the tree and loop descendants of keystones. Thus, looking at (5.2) and nothing else we iteratively reconstruct the entire RG-closed set of operators generated by the given keystone ones.

\subsection{Hierarachy/tower of Virasoro-like constraints}

Moreover, the same procedure can be promoted to the level of Virasoro-like identities, i.e. for the generating functions of recursion relations.

Step 1. To this end, we can start from a single keystone operator $\mathcal{K}_{2}$, discover that the recursion generates from it all $\mathcal{K}_{k}$ with arbitrary $k$ as the tree operators, while all loop operators appear algebraic function of those. Thus, at this stage, one needs to add all these $\mathcal{K}_{k}$ to extend the action with independent coefficients (time-variables) $t_{k}$, i.e. to obtain the extended red model (4.2). The above reasoning is now expressed in form of the basic level Virasoro constraint (4.4), which we naturally call "red-Virasoro".

Step 2. We could instead begin from another keystone operator $\mathcal{K}_{2}$ and arrive at the green model satisfying the green-Virasoro constraints.

Step 3. What we need, is the model where both $\mathcal{K}_{2}$ and $\mathcal{K}_{2}$ are included as keystones, and the natural starting point for the extended action is the one in (5.1). However, the red-Virasoro constraints hold for this model only at the point where all green times $t_{k}=0$, while the green-Virasoro constraints hold at the point where all $t_{k}=0$. When both types of time-variables are non-vanishing, we should include the descendants coming from the recursion relation (5.2) with $\mathcal{K}=\mathcal{K}_{m} \mathcal{K}_{n}$, i.e. the operators $\mathcal{K}_{m, n}$. Since they are 
algebraically independent of $\mathcal{K}_{k}$ and $\mathcal{K}_{k}$, we should add them to extended the action with their own new couplings (time-variables) $t_{m, n}$. Then, in the Aristotelian model (5.1), we immediately obtain the new Virasoro constraint of the next level

$$
\begin{aligned}
& \left(-\mu \frac{\partial}{\partial t_{m+1}}+\sum_{k} k t_{k} \frac{\partial}{\partial t_{k+m}}+\underline{\sum_{k} k t_{k} \frac{\partial}{\partial t_{m+1, k}}}+\right. \\
& +\sum_{a=1}^{m-1} \frac{\partial^{2}}{\partial t_{a} \partial t_{m-a}}+\underbrace{\left(N_{r}+N_{g} N_{b}\right)}_{\alpha}\left(1-\delta_{m, 0}\right) \frac{\partial}{\partial t_{m}}+\underbrace{N_{r} N_{g} N_{b}}_{\beta} \cdot \delta_{m, 0}) \mathcal{Z}\{t, t\}=0
\end{aligned}
$$

valid for arbitrary values of $t_{k}$ and $t_{n}$ (new as compared to (4.4) is just the underlined term). Eq. (5.3) is the Ward identity for the shift $\delta \bar{A}=\partial \mathcal{K}_{m} / \partial A$. Of course, (5.3) has a "green" counterpart associated with the shift $\delta \bar{A}=\partial \mathcal{K}_{n} / \partial A$ :

$$
\begin{aligned}
& \left(-\mu \frac{\partial}{\partial t_{n+1}}+\sum_{k} k t_{k} \frac{\partial}{\partial t_{k+n}}+\sum_{k} k t_{k} \frac{\partial}{\partial t_{k, n+1}}\right. \\
& +\sum_{a=1}^{n-1} \frac{\partial^{2}}{\partial t_{a} \partial t_{n-a}}+\underbrace{\left(N_{r}+N_{g} N_{b}\right)}_{\alpha}\left(1-\delta_{n, 0}\right) \frac{\partial}{\partial t_{n}}+\underbrace{N_{r} N_{g} N_{b}}_{\beta} \cdot \delta_{n, 0}) \mathcal{Z}\{t, t\}=0
\end{aligned}
$$

These two sets of constraints taken at all zero times $t_{k}$ 's lead to the relations for the correlators that we already know from the previous section:

$$
\begin{array}{r}
-\mu\left\langle\mathcal{K}_{m+1}\right\rangle+\sum_{a=1}^{m-1}\left\langle\mathcal{K}_{a} \mathcal{K}_{m-a}\right\rangle+\alpha\left(1-\delta_{m, 0}\right)\left\langle\mathcal{K}_{m}\right\rangle+\beta \cdot \delta_{m, 0}=0 \\
-\mu\left\langle\mathcal{K}_{n+1}\right\rangle+\sum_{a=1}^{n-1}\left\langle\mathcal{K}_{a} \mathcal{K}_{n-a}\right\rangle+\alpha\left(1-\delta_{n, 0}\right)\left\langle\mathcal{K}_{n}\right\rangle+\beta \cdot \delta_{n, 0}=0
\end{array}
$$

However, one can go further and take the first derivative of (5.3) w.r.t. $t_{p}$ at all zero times $t_{k}$ 's which gives

$$
-\mu\left\langle\mathcal{K}_{m} \mathcal{K}_{p}\right\rangle+p\left\langle\mathcal{K}_{m, p}\right\rangle+\sum_{a=1}^{m-2}\left\langle\mathcal{K}_{a} \mathcal{K}_{m-a-1} \mathcal{K}_{p}\right\rangle+\alpha\left(1-\delta_{m, 1}\right)\left\langle\mathcal{K}_{m-1} \mathcal{K}_{p}\right\rangle+\beta \cdot\left\langle\mathcal{K}_{p}\right\rangle \cdot \delta_{m, 1}=0
$$

Similarly, the derivative of (5.4) w.r.t. $t_{q}$ gives

$$
-\mu\left\langle\mathcal{K}_{n} \mathcal{K}_{q}\right\rangle+q\left\langle\mathcal{K}_{n, q}\right\rangle+\sum_{a=1}^{n-2}\left\langle\mathcal{K}_{a} \mathcal{K}_{n-a-1} \mathcal{K}_{q}\right\rangle+\alpha\left(1-\delta_{n, 1}\right)\left\langle\mathcal{K}_{n-1} \mathcal{K}_{q}\right\rangle+\beta \cdot\left\langle\mathcal{K}_{q}\right\rangle \cdot \delta_{n, 1}=0
$$


Choosing in these expressions $q=m$ and $p=n$ and adding them with the coefficients $m$ and $n$ respectively, one arrives at the recurrent relation (we take into account that $\mathcal{K}_{n, q}=\mathcal{K}_{q, n}$ )

$$
\begin{aligned}
- & (m+n) \mu \cdot\left\langle\mathcal{K}_{m} \mathcal{K}_{n}\right\rangle+2 m n \cdot\left\langle\mathcal{K}_{m, n}\right\rangle+m \alpha\left(1-\delta_{m, 1}\right) \cdot\left\langle\mathcal{K}_{m-1} \mathcal{K}_{n}\right\rangle \\
+ & m \cdot \sum_{\substack{m_{1}, m_{2} \geq 1 \\
m_{1}+m_{2}=m-1}}\left\langle\mathcal{K}_{m_{1}} \mathcal{K}_{m_{2}} \mathcal{K}_{n}\right\rangle+n \alpha\left(1-\delta_{n, 1}\right) \cdot\left\langle\mathcal{K}_{m} \mathcal{K}_{n-1}\right\rangle \\
+ & n \cdot \sum_{\substack{n_{1}, n_{2} \geq 1 \\
n_{1}+n_{2}=n-1}}\left\langle\mathcal{K}_{m} \mathcal{K}_{n_{1}} \mathcal{K}_{n_{2}}\right\rangle+\beta \cdot\left\langle\mathcal{K}_{n}\right\rangle \cdot \delta_{m, 1}+\beta \cdot\left\langle\mathcal{K}_{m}\right\rangle \cdot \delta_{n, 1}=0
\end{aligned}
$$

In fact, using $(5.3)$ or (5.4), one can easily calculate the correlators $\left\langle\mathcal{K}_{m, n}\right\rangle$ in the planar limit. The simplest way to do this is to get the equation for the generating functions. This is done immediately, however, requires first evaluating the two-resolvents in the planar limit. This calculation will be presented elsewhere, and, in the next section, we perform just direct calculation through the Gaussian integration.

Next steps: (5.3) holds, however, only at vanishing values of the newly-added times: $t_{m, n}=0$. If we want to release these time-variables, we will need new operators to add, include them with new couplings and get the Virasoro-like constraints of the next level, and so on. In this way, we arrive at the clearly ordered tower of embedded constraints, which is generically infinite, though can sometimes terminate, if the newly emerging operators get algebraically dependent of the previous ones. This unavoidably happens, for example, if we keep the values of $N_{r}, N_{g}$ and $N_{b}$ fixed and integer. This option was not considered interesting in the simplest matrix models, where much more can be achieved: a single generating function for all Ward identities at all $N$. However, for the tensor models this option can turn out to be much more interesting.

Possible culmination will appear when one manages to understand this entire wellstructured tower of constraints well enough and find a top level generating function(al) unifying them all. There is, however, a long way to go before we reach this point, and one of the ways is a reformulation of particular models in the BZ terms summarized in above section 3 .

Advantages of reformulation in terms of Virasoro-like constraints are not exhausted by their beauty: important is an ability to go beyond the Gaussian phases and to true non-perturbative considerations. We briefly mentioned in section 2 possible techniques involved in this part of the story, and they should be also looked at and for in the study of tensor models.

In the remaining part of this section, we come back down to earth and start doing the first of above steps for the Aristotelian model, the first non-trivial one among the tensor models. Our main concern is developing some technique for the Gaussian calculations. The first results are tested with the basic recursions (5.2) and (5.8). The main goals, however, are the lifting from the recursions to their generating functions and functionals and the search for general formulas and relations like (2.55). 


\subsection{Gaussian averages in the Aristotelian model by direct computation}

\subsubsection{Red rings and green rings}

Like in the red model from section 4 , when only operators $\mathcal{K}_{m}$ are present in the correlators, they can be considered as those in the rectangular matrix model of size $N_{b} \times N_{g} N_{b}$, i.e. taken just from the table in section 2.3.2 with $\alpha=N_{r}+N_{g} N_{b}$ and $\beta=N_{r} N_{g} N_{b}$ :

$$
\left\langle\prod_{i} \mathcal{K}_{m_{i}}\right\rangle=\mathcal{O}_{[\Lambda]}^{N_{b} \times N_{g} N_{b}}
$$

for $\Lambda=\left[m_{1} \geq m_{2} \geq \ldots \geq 0\right]$.

The same is true when only green operators are present:

$$
\left\langle\prod_{j} \mathcal{K}_{n_{j}}\right\rangle=\mathcal{O}_{[\Lambda]}^{N_{g} \times N_{r} N_{b}}
$$

only this time $\alpha=N_{g}+N_{r} N_{b}$ and $\Lambda=\left[n_{1} \geq n_{2} \geq \ldots \geq 0\right]$, while $\beta=N_{r} N_{g} N_{b}$ remains the same.

The operators $\mathcal{K}_{m}$ and $\mathcal{K}_{n}$ are naturally depicted as red and green circles of lengths $m$ and $n$ respectively, as will be more accurately explained in section 5.4 below. Since $\mathcal{K}_{1}=\mathcal{K}_{1}$, the circles of unit length can be considered either red or green. In the rectangular matrix model, the insertion of such unit circles changes averages in a very simple way described by the factorization formula (4.14).

\subsubsection{Reductions at $N=1$}

In more complicated cases, the averages in the Aristotelian model (5.1) are not reduced to those for the rectangular matrices. However, they do so, when any of the three colorings disappear, i.e. when any one of the three numbers $N_{r}, N_{g}$ or $N_{b}$ becomes unity. This provides a convenient check for formulas and also helps to build them by lifting from the three different rectangular model limits: such calculations can provide the answers modulo $\left(N_{r}^{2}-1\right)\left(N_{g}^{2}-1\right)\left(N_{b}^{2}-1\right)$. We illustrate this approach in examples below in this section.

\subsubsection{Red and green rings together}

The next correlators to look at are the collections of rings of different colors, beginning from $\left\langle\mathcal{K}_{m} \mathcal{K}_{n}\right\rangle$.

First of all, if we put, say, $N_{g}=1$ then the operators $\mathcal{K}_{m}$ and $\mathcal{K}_{n}$ turn respectively into $\mathcal{K}_{m}, \mathcal{K}_{1}^{n}$ of the rectangular $N_{r} \times N_{b}$ matrix model, i.e. will be easily calculated with the help of (4.14):

$$
\left\langle\mathcal{K}_{m} \mathcal{K}_{n}\right\rangle \stackrel{N_{g}=1}{=} \mathcal{O}_{\left[m, 1^{n}\right]}^{N_{r} \times N_{b}}=\frac{1}{\mu^{n}} \mathcal{O}_{[m]}^{N_{r} \times N_{b}} \prod_{j=0}^{n-1}\left(N_{r} N_{b}+m+j\right)
$$

Likewise,

$$
\left\langle\mathcal{K}_{m} \mathcal{K}_{n}\right\rangle \stackrel{N_{r}=1}{=} \mathcal{O}_{\left[n, 1^{m}\right]}^{N_{g} \times N_{b}}=\frac{1}{\mu^{n}} \mathcal{O}_{[n]}^{N_{g} \times N_{b}} \prod_{i=0}^{m-1}\left(N_{g} N_{b}+n+i\right)
$$


If $N_{b}=1$, then the both types of operators turn into the circles in the rectangular model $N_{r} \times N_{g}$ and

$$
\left\langle\mathcal{K}_{m} \mathcal{K}_{n}\right\rangle \stackrel{N_{b}=1}{=} \mathcal{O}_{[m, n]}^{N_{r} \times N_{g}}
$$

where this time we should substitute $\alpha=\alpha \equiv N_{r}+N_{g}$. As already mentioned, these formulas provide a nice starting point for evaluating the correlator at generic values of $N_{r}$, $N_{g}$ and $N_{b}$.

For example,

$$
\begin{aligned}
& \left\langle\mathcal{K}_{2} \mathcal{K}_{2}\right\rangle \\
& =\frac{N_{r} N_{g} N_{b}}{\mu^{4}}\left(N_{r}^{2} N_{g}^{2} N_{b}^{3}+\left(N_{r}^{2}+N_{g}^{2}+4\right) N_{r} N_{g} N_{b}^{2}+\left(N_{r}^{2} N_{g}^{2}+4 N_{r}^{2}+4 N_{g}^{2}+2\right) N_{b}+6 N_{r} N_{g}\right)
\end{aligned}
$$

and

$$
\begin{aligned}
\left\langle\mathcal{K}_{3} \mathcal{K}_{2}\right\rangle= & \frac{N_{r} N_{g} N_{b}}{\mu^{5}}\left(N_{r}^{2} N_{g}^{3} N_{b}^{4}+\left(3 N_{r}^{2}+N_{g}^{2}+6\right) N_{r} N_{g}^{2} N_{b}^{3}+\right. \\
& +\left(N_{r}^{4}+3 N_{r}^{2} N_{g}^{2}+19 N_{r}^{2}+6 N_{g}^{2}+6\right) N_{g} N_{b}^{2} \\
& \left.+\left(N_{r}^{2} N_{g}^{2}+6 N_{r}^{2}+25 N_{g}^{2}+18\right) N_{r} N_{b}+12\left(N_{r}^{2}+1\right) N_{g}\right)
\end{aligned}
$$

are fully defined by the three reductions (5.11)-(5.13). The matrix model calculation becomes insufficient beginning from

$$
\begin{aligned}
\left\langle\mathcal{K}_{4} \mathcal{K}_{2}\right\rangle= & \frac{N_{r} N_{g} N_{b}}{\mu^{6}}\left(N_{r}^{2} N_{g}^{4} N_{b}^{5}+\left(6 N_{r}^{2}+N_{g}^{2}+8\right) N_{r} N_{g}^{3} N_{b}^{4}+\right. \\
& +\left(6 N_{r}^{4}+6 N_{r}^{2} N_{g}^{2}+53 N_{r}^{2}+8 N_{g}^{2}+12\right) N_{g}^{2} N_{b}^{3} \\
& +\left(N_{r}^{4}+6 N_{r}^{2} N_{g}^{2}+53 N_{r}^{2}+65 N_{g}^{2}+100\right) N_{r} N_{g} N_{b}^{2}+ \\
& \left.+\left(N_{r}^{4} N_{g}^{2}+8 N_{r}^{4}+85 N_{r}^{2} N_{g}^{2}+80 N_{r}^{2}+68 N_{g}^{2}+32\right) N_{b}+20\left(N_{r}^{2}+5\right) N_{r} N_{g}\right)
\end{aligned}
$$

and

$$
\begin{aligned}
\left\langle\mathcal{K}_{3} \mathcal{K}_{3}\right\rangle= & \frac{N_{r} N_{g} N_{b}}{\mu^{6}}\left(N_{r}^{3} N_{g}^{3} N_{b}^{5}+3\left(N_{r}^{2}+N_{g}^{2}+3\right) N_{r}^{2} N_{g}^{2} N_{b}^{4}+\right. \\
& +\left(N_{r}^{4}+9 N_{r}^{2} N_{g}^{2}+N_{g}^{4}+28 N_{r}^{2}+28 N_{g}^{2}+18\right) N_{r} N_{g} N_{b}^{3}+ \\
& +3\left(N_{r}^{4} N_{g}^{2}+N_{r}^{2} N_{g}^{4}+3 N_{r}^{4}+35 N_{r}^{2} N_{g}^{2}+3 N_{g}^{4}+15 N_{r}^{2}+15 N_{g}^{2}+2\right) N_{b}^{2}+ \\
& \left.+\left(N_{r}^{2} N_{g}^{2}+46 N_{r}^{2}+46 N_{g}^{2}+181\right) N_{r} N_{g} N_{b}+30\left(N_{r}^{2}+1\right)\left(N_{g}^{2}+1\right)\right)
\end{aligned}
$$

From these examples, supplemented by other corollaries of (5.11)-(5.13), one can observe an emerging structure of the answer:

$$
\begin{aligned}
\left\langle\mathcal{K}_{m} \mathcal{K}_{2}\right\rangle= & \frac{N_{r} N_{g} N_{b}}{\mu^{m+1}}\left\{N_{r}^{2} N_{g}^{m} N_{b}^{m+1}+\left(\frac{m(m-1)}{2} N_{r}^{2}+N_{g}^{2}+2 m\right) N_{r} N_{g}^{m-1} N_{b}^{m}+\right. \\
& +\left(\frac{m(m-1)^{2}(m-2)}{12} \cdot N_{r}^{4}+\frac{m(m-1)}{2} N_{r}^{2} N_{g}^{2}+\frac{m(m-1)\left(m^{2}+23 m-2\right)}{24}\right. \\
& \left.\left.\cdot N_{r}^{2}+2 m N_{g}^{2}+m(m-1)\right) N_{g}^{m-2} N_{b}^{m-1}++O\left(N_{b}^{m-2}\right)\right\}
\end{aligned}
$$


and, further,

$$
\begin{aligned}
\left\langle\mathcal{K}_{m} \mathcal{K}_{n}\right\rangle \\
=\frac{N_{r} N_{g} N_{b}}{\mu^{m+1}}\left\{N_{r}^{n} N_{g}^{m} N_{b}^{m+n-1}+\left(\frac{m(m-1)}{2} N_{r}^{2}+\frac{n(n-1)}{2} N_{g}^{2}+m n\right) N_{r}^{n-1} N_{g}^{m-1} N_{b}^{m+n-2}+\right. \\
\quad+\left(\frac{m(m-1)^{2}(m-2)}{12} \cdot N_{r}^{4}+\frac{m(m-1)}{2} \frac{n(n-1)}{2} \cdot N_{r}^{2} N_{g}^{2}+\frac{n(n-1)^{2}(n-2)}{12} \cdot N_{g}^{4}+\right. \\
\quad+\frac{m(m-1) \cdot((m+1)(m-2)+12 m n)}{24} N_{r}^{2}+\frac{n(n-1) \cdot((n+1)(n-2)+12 m n)}{24} N_{g}^{2}+ \\
\left.\left.\quad+\frac{m(m-1) n(n-1)}{2}\right) N_{r}^{n-2} N_{g}^{m-2} N_{b}^{m+n-3}+O\left(N_{b}^{m+n-4}\right)\right\}
\end{aligned}
$$

but more data is needed to fully reconstruct it even for this simplest kind of Gaussian correlators in the simplest of tensor models.

As usual, many terms in the expansion (5.19) can be restored from the reduction to $N_{g}=1$, when it should match the large- $N_{b}$ expansion

$$
\begin{aligned}
& \mathcal{O}_{\left[m, 1^{n}\right]}^{N_{r} \times N_{b}}=\mathcal{O}_{[m]}^{N_{r} \times N_{b}} \prod_{i=m}^{m+n-1}\left(N_{r} N_{b}+i\right)= \\
&=N_{r}^{n} N_{b}^{m+n-1}\left(1+\frac{m(m-1)}{2} \frac{N_{r}}{N_{b}}+\frac{m(m-1)^{2}(m-2)}{12} \frac{N_{r}^{2}}{N_{b}^{2}}+\frac{(m+1) m(m-1)(m-2)}{N_{b}^{2}}+\ldots\right) . \\
& \cdot\left(1+\frac{(2 m+n-1) n}{2 N_{r} N_{b}}+\frac{n(n-1)\left(12 m^{2}+12 m n+3 n^{2}-12 m-7 n+2\right)}{24 N_{r}^{2} N_{b}^{2}}+\ldots\right)
\end{aligned}
$$

Efficient handling of such formulas requires an adequate multi-color generalization of the FT calculus, which will be developed elsewhere.

\subsubsection{Direct evaluation of $\left\langle\mathcal{K}_{2,2}\right\rangle$}

In our notation, the operators $\mathcal{K}_{m, 1}=\mathcal{K}_{m}$, thus, their Gaussian averages are just the same as in (4.9). The averages of $\mathcal{K}_{1, n}=\mathcal{K}_{n}$ are obtained by the substitution of $\alpha=N_{r}+N_{g} N_{b}$ by $\alpha=N_{g}+N_{r} N_{b}$.

Thus, the first non-trivial example is the average of $\mathcal{K}_{2,2}$ :

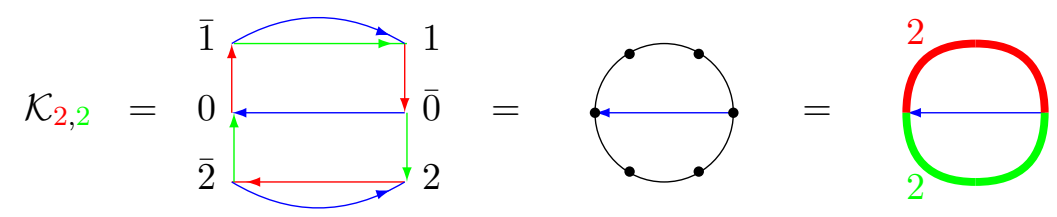

By the Wick theorem, this average is a sum of six different pairings

$$
\left\langle\mathcal{K}_{2,2}\right\rangle=\frac{N_{r} N_{g} N_{b}}{\mu^{3}}(\underbrace{N_{r} N_{g} N_{b}^{2}}_{<0 \overline{0}><1 \overline{1}><2 \overline{2}>}+\overbrace{N_{r}^{2} N_{b}}^{<0 \overline{0}}+\underbrace{N_{g}^{2} N_{b}}_{<0 \overline{2}><1 \overline{1}><2 \overline{0}>}+\overbrace{<0 \overline{1}><1 \overline{2}><2 \overline{0}>+<0 \overline{2}><1 \overline{0}><2 \overline{1}>}^{<0 \overline{0}><1 \overline{2}><2 \overline{1}>})
$$


An alternative calculation uses the recursion relation i.e. the Virasoro constraint at $t=0$. Namely, eq. (5.2) in this case says

$$
3 \mu\left\langle\mathcal{K}_{2,2}\right\rangle=\mu\left\langle A_{i_{r} \alpha_{b}}^{j_{g}} \frac{\partial \mathcal{K}_{2,2}}{\partial A_{i_{r} \alpha_{b}}^{j_{g}}}\right\rangle=\left\langle\frac{\partial^{2} \mathcal{K}_{2,2}}{\partial A_{i_{r} \alpha_{b}}^{j_{g}} \partial \bar{A}_{j_{g}}^{i_{r} \alpha_{b}}}\right\rangle=\sum_{a, b=0}^{2}\left\langle\frac{\partial^{2} \mathcal{K}_{2,2}}{\partial A(a) \partial \bar{A}(\bar{b})}\right\rangle
$$

where the sum goes over pairs of vertices labeled by $0,1,2$ and $\overline{0}, \overline{1}, \overline{2}$ in the picture. Explicitly contracting the indices in these 9 terms, one obtains

$$
\begin{aligned}
& 3 \mu\left\langle\mathcal{K}_{2,2}\right\rangle= \underbrace{\left(2 N_{r}+N_{g} N_{b}\right)\left\langle\mathcal{K}_{2}\right\rangle}_{<0 \overline{1}>,<1 \overline{0}>,<1 \overline{1}>}+\overbrace{\left(2 N_{g}+N_{r} N_{b}\right)\left\langle\mathcal{K}_{2}\right\rangle}^{<0 \overline{2}>,<2 \overline{0}>,<2 \overline{2}>}+\underbrace{2\left\langle\mathcal{K}_{2}\right\rangle}_{<1 \overline{2}>,<2 \overline{1}>}+\overbrace{N_{b}\left\langle\mathcal{K}_{1} \mathcal{K}_{1}\right\rangle}^{<0 \overline{0}>}= \\
& \stackrel{(4.9)}{=} \frac{N_{r} N_{g} N_{b}}{\mu^{2}}\left(\left(2 N_{r}+N_{g} N_{b}\right)\left(N_{r} N_{b}+N_{g}\right)+\left(2 N_{g}+N_{r} N_{b}\right)\left(N_{g} N_{b}+N_{r}\right)+\right. \\
&\left.\quad+2\left(N_{r} N_{g}+N_{b}\right)+N_{b}\left(N_{r} N_{g} N_{b}+1\right)\right)= \\
&=\frac{3 N_{r} N_{g} N_{b}}{\mu^{2}}\left(N_{r} N_{g} N_{b}^{2}+N_{r}^{2} N_{b}+N_{g}^{2} N_{b}+2 N_{r} N_{g}+N_{b}\right)
\end{aligned}
$$

in full accordance with (5.21). We remind that there is a symmetry between $N_{r}$ and $N_{g}$, but not $N_{b}$.

A specifics (simplicity) of this example is that the next-level operator with more blue lines (two in this case) arising in the recursion appeared to be equivalent to what we denoted as $\mathcal{K}_{2}$, for which we actually now the answer: its average is the same $\alpha \beta / \mu^{2}$ as that of $\mathcal{K}_{2}$, with $\alpha=N_{r}+N_{g} N_{b}$ substituted by $\alpha=N_{b}+N_{r} N_{g}$.

The FT generating function is remarkably simple in this case $\mathcal{K}_{2,2}$ :

$$
\sum_{N_{r}, N_{g}, N_{b}} \lambda_{r}^{N_{r}} \lambda_{g}^{N_{g}} \lambda_{b}^{N_{b}} \cdot\left\langle\mathcal{K}_{2,2}^{N_{r} \times N_{g} \times N_{b}}\right\rangle=\frac{6 \lambda_{r} \lambda_{g} \lambda_{b}}{\left(1-\lambda_{r}\right)^{4}\left(1-\lambda_{g}\right)^{4}\left(1-\lambda_{b}\right)^{4}}\left(\left(1-\lambda_{r} \lambda_{g}\right)^{2}-\left(\lambda_{r}-\lambda_{g}\right)^{2} \lambda_{b}{ }^{2}\right)
$$

but it becomes sophisticated for averages with higher $m$ and $n$.

\subsubsection{Some other Gaussian averages $\left\langle\mathcal{K}_{m, n}\right\rangle$ and their reductions}

At $N_{g}=1$ the average of $\mathcal{K}_{m, n}$ becomes equivalent to that of $\operatorname{Tr}(M \bar{M})^{m}(\operatorname{Tr} M \bar{M})^{n-1}$ in the rectangular matrix model with the matrix size $N_{r} \times N_{b}$. For $N_{r}=1$, the same is true for the $N_{g} \times N_{b}$ model. Finally, at $N_{b}=1$ we get the equivalence to just $\operatorname{Tr}(M \bar{M})^{m+n-1}$ in the $N_{r} \times N_{g}$ model. Thus, we get the following expressions through the single-hook averages:

$$
\begin{gathered}
\left\langle\mathcal{K}_{m, n}\right\rangle \stackrel{N_{g}=1}{=} \mathcal{O}_{\left[m, 1^{n-1}\right]}^{N_{r} \times N_{b}} \\
\left\langle\mathcal{K}_{m, n}\right\rangle \stackrel{N_{r}=1}{=} \mathcal{O}_{\left[n, 1^{m-1}\right]}^{N_{g} \times N_{b}} \\
\left\langle\mathcal{K}_{m, n}\right\rangle \stackrel{\stackrel{N_{b}=1}{=}}{\mathcal{O}_{[m+n-1}^{N_{r} \times N_{g}}}
\end{gathered}
$$


When only one of the three $N$ 's is different from unity, the rectangular model reduces to the vector one and we get a universal answer:

$$
\begin{aligned}
\left\langle\mathcal{K}_{m, n}\right\rangle \stackrel{N_{r}=N_{g}=1}{=} N_{b}\left(N_{b}+1\right)\left(N_{b}+2\right) \ldots\left(N_{b}+m+n-1\right)=\frac{\left(N_{b}+m+n-1\right) !}{\left(N_{b}-1\right) !} \\
\left\langle\mathcal{K}_{m, n}\right\rangle \stackrel{N_{r}=N_{b}=1}{=} N_{g}\left(N_{g}+1\right)\left(N_{g}+2\right) \ldots\left(N_{g}+m+n-1\right)=\frac{\left(N_{g}+m+n-1\right) !}{\left(N_{g}-1\right) !} \\
\left\langle\mathcal{K}_{m, n}\right\rangle \stackrel{N_{g}=N_{b}=1}{=} N_{r}\left(N_{r}+1\right)\left(N_{r}+2\right) \ldots\left(N_{r}+m+n-1\right)=\frac{\left(N_{r}+m+n-1\right) !}{\left(N_{r}-1\right) !}
\end{aligned}
$$

With the help of (4.9), one can check that (5.25) is indeed true:

$$
\begin{array}{r}
\left\langle\mathcal{K}_{2,2}\right\rangle=\frac{N_{r} N_{g} N_{b}}{\mu^{3}}\left(N_{r} N_{g} N_{b}^{2}+\left(N_{r}^{2}+N_{g}^{2}+1\right) N_{b}+2 N_{r} N_{g}\right) \\
N_{g}=1 \quad N_{r} N_{b}\left(N_{r}+N_{b}\right)\left(N_{r} N_{b}+2\right)=\mathcal{O}_{[2,1]}^{N_{r} \times N_{b}} \\
N_{b}=1 \quad N_{r} N_{g}\left(N_{r}^{2}+N_{g}^{2}+3 N_{r} N_{g}+1\right)=\mathcal{O}_{[3]}^{N_{r} \times N_{g}}
\end{array}
$$

- Similarly for $\mathcal{K}_{3,2}$ we get:

$$
\begin{aligned}
& \left\langle\mathcal{K}_{3,2}\right\rangle=\frac{N_{r} N_{g} N_{b}}{\mu^{4}}\left(N_{r} N_{g}^{2} N_{b}^{3}+\left(3 N_{r}^{2}+N_{g}^{2}+2\right) N_{g} N_{b}^{2}+\left(N_{r}^{2}+5 N_{g}^{2}+5\right) N_{r} N_{b}+3\left(N_{r}^{2}+1\right) N_{g}\right) \\
& N_{g}=1 \quad N_{r} N_{b}\left(N_{r} N_{b}+3\right)\left(N_{r}^{2}+N_{b}^{2}+3 N_{r} N_{b}+1\right)=\mathcal{O}_{[3,1]}^{N_{r} \times N_{b}} \\
& N_{r}=1 \quad N_{g} N_{b}\left(N_{g}+N_{b}\right)\left(N_{g} N_{b}+2\right)\left(N_{g} N_{b}+3\right)=\mathcal{O}_{[2,1,1]}^{N_{g} \times N_{b}} \\
& N_{b}=1 \quad N_{r} N_{g}\left(N_{r}+N_{g}\right)\left(N_{r}^{2}+N_{g}^{2}+5 N_{r} N_{g}+5\right)=\mathcal{O}_{[4]}^{N_{r} \times N_{g}}
\end{aligned}
$$

Note that for $N_{b} \neq 1$ there is no symmetry between $N_{r}$ and $N_{g}$ in this case: by exchanging $N_{r}$ and $N_{g}$, one gets the expression for $\left\langle\mathcal{K}_{2,3}\right\rangle$. In particular, in this case, we would get

$$
\begin{gathered}
\left\langle\mathcal{K}_{2,3}\right\rangle=\frac{N_{r} N_{g} N_{b}}{\mu^{4}}\left(N_{r}^{2} N_{g} N_{b}^{3}+\left(N_{r}^{2}+3 N_{g}^{2}+2\right) N_{r} N_{b}^{2}+\left(5 N_{r}^{2}+N_{g}^{2}+5\right) N_{g} N_{b}+3\left(N_{g}^{2}+1\right) N_{r}\right) \\
N_{g}=1 \quad N_{r} N_{b}\left(N_{r}+N_{b}\right)\left(N_{r} N_{b}+2\right)\left(N_{r} N_{b}+3\right) \quad=\mathcal{O}_{[2,1,1]}^{N_{r} \times N_{b}} \\
N_{r}=1 \quad N_{g} N_{b}\left(N_{g} N_{b}+3\right)\left(N_{g}^{2}+N_{b}^{2}+3 N_{g} N_{b}+1\right)=\mathcal{O}_{[3,1]}^{N_{g} \times N_{b}}
\end{gathered}
$$


- For $\mathcal{K}_{3,3}$ we obtain:

$$
\begin{aligned}
& \mathcal{K}_{3,3}={ }_{2}= \\
& \left\langle\mathcal{K}_{3,3}\right\rangle=\frac{N_{r} N_{g} N_{b}}{\mu^{5}}\left(N_{r}^{2} N_{g}^{2} N_{b}^{4}+\left(3 N_{r}^{2}+3 N_{g}^{2}+4\right) N_{r} N_{g} N_{b}^{3}+\right. \\
& \left.+\left(N_{r}^{4}+N_{g}^{4}+13 N_{r}^{2} N_{g}^{2}+9 N_{r}^{2}+9 N_{g}^{2}+2\right) N_{b}^{2}+\left(7 N_{r}^{2}+7 N_{g}^{2}+36\right) N_{r} N_{g} N_{b}+6\left(N_{r}^{2}+1\right)\left(N_{g}^{2}+1\right)\right) \\
& N_{g}=1 \quad N_{r} N_{b}\left(N_{r} N_{b}+3\right)\left(N_{r} N_{b}+4\right)\left(N_{r}^{2}+N_{b}^{2}+3 N_{b} N_{r}+1\right) \quad=\mathcal{O}_{[3,1,1]}^{N_{r} \times N_{b}} \\
& N_{b}=1 \quad N_{r} N_{g}\left(N_{r}^{4}+10 N_{r}^{3} N_{g}+20 N_{r}^{2} N_{g}^{2}+10 N_{r} N_{g}^{3}+N_{g}^{4}+15 N_{r}^{2}+40 N_{r} N_{g}+15 N_{g}^{2}+8\right)=\mathcal{O}_{[5]}^{N_{r} \times N_{g}} \\
& \left\langle\mathcal{K}_{4,2}\right\rangle=\frac{N_{r} N_{g} N_{b}}{\mu^{5}}\left(N_{r} N_{g}^{3} N_{b}^{4}+\left(6 N_{r}^{2}+N_{g}^{2}+3\right) N_{g}^{2} N_{b}^{3}+\left(6 N_{r}^{2}+9 N_{g}^{2}+20\right) N_{r} N_{g} N_{b}^{2}+\right. \\
& \left.+\left(N_{r}^{4}+14 N_{r}^{2} N_{g}^{2}+15 N_{r}^{2}+12 N_{g}^{2}+8\right) N_{b}+4 N_{r} N_{g}\left(N_{r}^{2}+5\right)\right) \\
& \begin{array}{ccrl}
N_{g}=1 & N_{r} N_{b}\left(N_{r} N_{b}+4\right)\left(N_{r}^{2}+N_{b}^{2}+5 N_{r} N_{b}+5\right) & =\mathcal{O}_{[4,1]}^{N_{r} \times N_{b}} \\
N_{r}=1 & N_{g} N_{b}\left(N_{g}+N_{b}\right)\left(N_{g} N_{b}+2\right)\left(N_{g} N_{b}+3\right)\left(N_{g} N_{b}+4\right) & =\mathcal{O}_{[2,1,1,1]}^{N_{g} \times N_{b}} \\
N_{b}=1 & N_{r} N_{g}\left(N_{r}^{4}+10 N_{r}^{3} N_{g}+20 N_{r}^{2} N_{g}^{2}+10 N_{r} N_{g}^{3}+N_{g}^{4}+15 N_{r}^{2}+40 N_{r} N_{g}+15 N_{g}^{2}+8\right) & =\mathcal{O}_{[5]}^{N_{r} \times N_{g}}
\end{array}
\end{aligned}
$$

Again, for $N_{b} \neq 1$ there is no symmetry between $N_{r}$ and $N_{g}$ in this case: by exchanging $N_{r}$ and $N_{g}$, one gets the expression for $\left\langle\mathcal{K}_{2,4}\right\rangle$.

$$
\begin{array}{cccc}
\left\langle\mathcal{K}_{4,3}\right\rangle= & \frac{N_{r} N_{g} N_{b}}{\mu^{6}}\left(N_{r}^{2} N_{g}^{3} N_{b}^{5}+3\left(2 N_{r}^{2}+N_{g}^{2}+2\right) N_{r} N_{g}^{2} N_{b}^{4}+\right. & \\
& +\left(6 N_{r}^{4}+N_{g}^{4}+24 N_{r}^{2} N_{g}^{2}+35 N_{r}^{2}+13 N_{g}^{2}+6\right) N_{g} N_{b}^{3}+ & \\
& +\left(N_{r}^{4}+12 N_{g}^{4}+34 N_{r}^{2} N_{g}^{2}+25 N_{r}^{2}+119 N_{g}^{2}+34\right) N_{r} N_{b}^{2}+ & \\
& \left.+\left(9 N_{r}^{4}+25 N_{r}^{2} N_{g}^{2}+140 N_{r}^{2}+22 N_{g}^{2}+78\right) N_{g} N_{b}+10\left(N_{r}^{2}+5\right)\left(N_{g}^{2}+1\right) N_{r}\right)
\end{array}
$$




\subsubsection{Wheel operators}

We consider also the wheel operators:

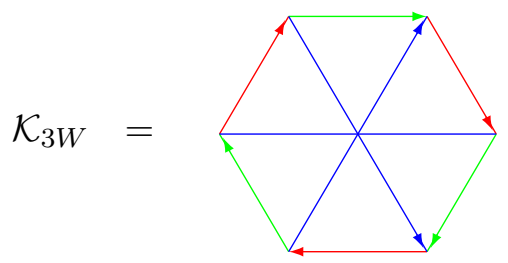

They are totally symmetric in the three colors and they can have only odd lengths $m$. The first non-trivial example after $\mathcal{K}_{1 W}=\mathcal{K}_{1}$ with the average $\left\langle\mathcal{K}_{1 W}\right\rangle=N_{r} N_{g} N_{b}$ and the FT function

$$
\sum_{N_{r}, N_{g}, N_{b}} \lambda_{r}^{N_{r}} \lambda_{g}^{N_{g}} \lambda_{b}^{N_{b}} \cdot\left\langle\mathcal{K}_{1 W}^{N_{r} \times N_{g} \times N_{b}}\right\rangle=\frac{\lambda_{r} \lambda_{g} \lambda_{b}}{\left(1-\lambda_{r}\right)^{2}\left(1-\lambda_{g}\right)^{2}\left(1-\lambda_{b}\right)^{2}}
$$

is

$$
\left\langle\mathcal{K}_{3 W}\right\rangle=\frac{N_{r} N_{g} N_{b}}{\mu^{3}}\left(3 N_{r} N_{g} N_{b}+N_{r}^{2}+N_{g}^{2}+N_{b}^{2}\right)
$$

Unfortunately, the FT formula is somewhat long in this case.

When any of the color numbers is one, this operators turns into an ordinary circle in the rectangular model with the remaining two colorings:

$$
\begin{array}{ll}
N_{g}=1 & \left\langle\mathcal{K}_{m W}\right\rangle=\mathcal{O}_{[m]}^{N_{r} \times N_{b}} \\
N_{r}=1 & \left\langle\mathcal{K}_{m W}\right\rangle=\mathcal{O}_{[m]}^{N_{g} \times N_{b}} \\
N_{b}=1 & \left\langle\mathcal{K}_{m W}\right\rangle=\mathcal{O}_{[m]}^{N_{r} \times N_{g}}
\end{array}
$$

Thus, the average of the wheel operators is the three-coloring continuation of the basic two-color averages in the first column of the table in section 2.3.2.

\subsubsection{Examples of recursion checks}

We have now enough explicit formulas to check the non-trivial example (5.8) of the recursion in the Aristotelian model. For example, for $m=2$ and $n=2$ we get:

$$
4 \mu \cdot\left\langle\mathcal{K}_{2} \mathcal{K}_{2}\right\rangle=8 \cdot\left\langle\mathcal{K}_{2,2}\right\rangle+2 \alpha \cdot \overbrace{\left\langle\mathcal{K}_{2} \mathcal{K}_{1}\right\rangle}^{\left\langle\mathcal{K}_{1} \mathcal{K}_{2}\right\rangle}+2 \alpha \cdot \overbrace{\left\langle\mathcal{K}_{2} \mathcal{K}_{1}\right\rangle}^{\left\langle\mathcal{K}_{2} \mathcal{K}_{1}\right\rangle}
$$

Note that the color of the unit circle operator $\mathcal{K}_{1}$ in the last two terms should be adjusted to that of $\mathcal{K}_{2}$, if one wants to apply the prescription from the end of section 5.3.1. Substituting the averages from $(5.14),(5.27)$ and (4.9) we obtain the identity (the overall factor $4 \beta \mu^{-3}$ is omitted):

$$
\begin{aligned}
& N_{r}^{2} N_{g}^{2} N_{b}^{3}+\left(N_{r}^{2}+N_{g}^{2}+4\right) N_{r} N_{g} N_{b}^{2}+\left(N_{r}^{2} N_{g}^{2}+4 N_{r}^{2}+4 N_{g}^{2}+2\right) N_{b}+6 N_{r} N_{g}= \\
& =2\left(N_{r} N_{g} N_{b}^{2}+\left(N_{r}^{2}+N_{g}^{2}+1\right) N_{b}+2 N_{r} N_{g}\right)+\left(N_{r}+N_{g} N_{b}\right)\left(N_{g}+N_{r} N_{b}\right)\left(N_{r} N_{g} N_{b}+2\right)
\end{aligned}
$$


Likewise, for $m=3$ and $n=2$

$$
5 \mu \cdot\left\langle\mathcal{K}_{3} \mathcal{K}_{2}\right\rangle=12 \cdot\left\langle\mathcal{K}_{3,2}\right\rangle+3 \alpha \cdot\left\langle\mathcal{K}_{2} \mathcal{K}_{2}\right\rangle+3 \cdot \overbrace{\left\langle\mathcal{K}_{2} \mathcal{K}_{1} \mathcal{K}_{1}\right\rangle}^{\left\langle\mathcal{K}_{1} \mathcal{K}_{1} \mathcal{K}_{2}\right\rangle}+2 \alpha \cdot \overbrace{\left\langle\mathcal{K}_{3} \mathcal{K}_{1}\right\rangle}^{\left\langle\mathcal{K}_{3} \mathcal{K}_{1}\right\rangle}
$$

what is indeed true:

$$
\begin{aligned}
& 5 \cdot\left(N_{r}^{2} N_{g}^{3} N_{b}^{4}+\left(3 N_{r}^{2}+N_{g}^{2}+6\right) N_{r} N_{g}^{2} N_{b}^{3}+\right. \\
& \left.+\left(N_{r}^{4}+3 N_{r}^{2} N_{g}^{2}+19 N_{r}^{2}+6 N_{g}^{2}+6\right) N_{g} N_{b}^{2}+\left(N_{r}^{2} N_{g}^{2}+6 N_{r}^{2}+25 N_{g}^{2}+18\right) N_{r} N_{b}+12\left(N_{r}^{2}+1\right) N_{g}\right)= \\
& =12 \cdot\left(N_{r} N_{g}^{2} N_{b}^{3}+\left(3 N_{r}^{2}+N_{g}^{2}+2\right) N_{g} N_{b}^{2}+\left(N_{r}^{2}+5 N_{g}^{2}+5\right) N_{r} N_{b}+3\left(N_{r}^{2}+1\right) N_{g}\right)+ \\
& +3 \cdot\left(N_{r}+N_{g} N_{b}\right)\left(N_{r}^{2} N_{g}^{2} N_{b}^{3}+\left(N_{r}^{2}+N_{g}^{2}+4\right) N_{r} N_{g} N_{b}^{2}+\left(N_{r}^{2} N_{g}^{2}+4 N_{r}^{2}+4 N_{g}^{2}+2\right) N_{b}+6 N_{r} N_{g}\right)+ \\
& +\left(N_{g}+N_{r} N_{b}\right)\left(N_{r} N_{g} N_{b}+3\right)\left(3 \cdot\left(N_{r} N_{g} N_{b}+2\right)+2 \cdot\left(\left(N_{r}+N_{g} N_{b}\right)^{2}+N_{r} N_{g} N_{b}+1\right)\right)
\end{aligned}
$$

When $N_{g}=1$, the operators $\mathcal{K}_{m}, \mathcal{K}_{n}$ and $\mathcal{K}_{m, n}$ turn respectively into $\mathcal{K}_{m}, \mathcal{K}_{1}^{n}$ and $\mathcal{K}_{m} \mathcal{K}_{1}^{n-1}$ of the $N_{r} \times N_{b}$ rectangular matrix model, and the recursion relation (5.8) reduces to

$$
\begin{aligned}
& (m+n) \mu \cdot \mathcal{O}_{\left[m, 1^{n}\right]}^{N_{r} \times N_{b}}=2 m n \cdot \mathcal{O}_{\left[m, 1^{n-1}\right]}^{N_{r} \times N_{b}}+ \\
& +m \alpha \cdot \mathcal{O}_{\left[m-1,1^{n}\right]}^{N_{r} \times N_{b}}+m \cdot \sum_{\substack{m_{1}, m_{2} \geq 1 \\
m_{1}+m_{2}=m-1}} \mathcal{O}_{\left[m_{1}, m_{2}, 1^{n}\right]}^{N_{r} \times N_{b}}+n \alpha \cdot \mathcal{O}_{\left[m, 1^{n-1}\right]}^{N_{r} \times N_{b}}+n(n-2) \cdot \mathcal{O}_{\left[m, 1^{n-1}\right]}^{N_{r} \times N_{b}}
\end{aligned}
$$

For example, at $n=1$ we get:

$$
(m+1) \mu \cdot \mathcal{O}_{[m, 1]}=(2 m+\beta) \cdot \mathcal{O}_{[m]}+m \alpha \cdot \mathcal{O}_{[m-1,1]}+m \cdot \sum_{\substack{m_{1}, m_{2} \geq 1 \\ m_{1}+m_{2}=m-1}} \mathcal{O}_{\left[m_{1}, m_{2}, 1\right]}
$$

(note that for $n=1$ the sum $\alpha+n-2=N_{r} N_{b}=\beta$ ). The factorization property (4.14) reduces this to $m(m+\beta-1)$ times the basic recursion of the rectangular complex model,

$$
\mu \cdot \mathcal{O}_{m}=\alpha \cdot \mathcal{O}_{m-1}+\sum_{\substack{m_{1}, m_{2} \geq 1 \\ m_{1}+m_{2}=m-1}} \mathcal{O}_{\left[m_{1}, m_{2}\right]}
$$

see section 4.6. The data from section 4.5 can be used to check other particular cases (5.40).

Similarly, at $N_{r}=1$ we have

$$
\begin{aligned}
& (m+n) \mu \cdot \mathcal{O}_{\left[n, 1^{m}\right]}^{N_{g} \times N_{b}}=2 m n \cdot \mathcal{O}_{\left[n, 1^{m-1}\right]}^{N_{g} \times N_{b}}+ \\
& \quad+m \alpha \cdot \mathcal{O}_{\left[n, 1^{m-1}\right]}^{N_{g} \times N_{b}}+m(m-2) \cdot \mathcal{O}_{\left[n, 1^{m-1}\right]}^{N_{g} \times N_{b}}+n \alpha \cdot \mathcal{O}_{\left[n, 1^{m-1}\right]}^{N_{g} \times N_{b}}+n \cdot \sum_{\substack{n_{1}, n_{2} \geq 1 \\
n_{1}+n_{2}=n-1}} \mathcal{O}_{\left[n_{1}, n_{2}, 1^{m-1}\right]}^{N_{g} \times N_{b}}
\end{aligned}
$$

For $N_{b}=1$ the operators $\mathcal{K}_{m}, \mathcal{K}_{n}$ and $\mathcal{K}_{m, n}$ turn into $\mathcal{K}_{m}, \mathcal{K}_{n}$ and $\mathcal{K}_{m+n-1}$ of the $N_{r} \times N_{g}$ model, and (5.8) becomes

$$
\begin{aligned}
(m+n) \mu \cdot \mathcal{O}_{[n, m]}^{N_{r} \times N_{g}} & =2 m n \cdot \mathcal{O}_{[m+n-1]}^{N_{r} \times N_{g}}+ \\
+m \alpha \cdot \mathcal{O}_{[m-1, n]}^{N_{r} \times N_{g}} & +m \cdot \sum_{\substack{m_{1}, m_{2} \geq 1 \\
m_{1}+m_{2}=m-1}} \mathcal{O}_{\left[m_{1}, m_{2}, n\right]}^{N_{r} \times N_{g}}+n \alpha \cdot \mathcal{O}_{[m, n-1]}^{N_{r} \times N_{g}}+n \cdot \sum_{\substack{n_{1}, n_{2} \geq 1 \\
n_{1}+n_{2}=n-1}} \mathcal{O}_{\left[m, n_{1}, n_{2}\right]}^{N_{r} \times N_{g}}
\end{aligned}
$$




\subsection{On RG completion of the Aristotelian model}

To study the problem of RG completion in this model, we introduce an additional notation.

The red and green propagators are

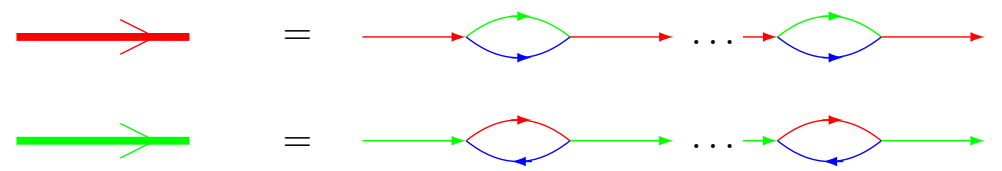

We denote them by the thick lines, but these are not propagators in Feynman diagrams, where the thick lines are multi-colored tubes/cables, here these are just the "dressed" thin-line trees.

The main operation in taking averages with the help of the Wick theorem is connecting two vertices by a Feynman propagator, i.e. just eliminating the two vertices and connecting the lines inside them.

If we consider a diagram which is a set of thick red and green lines meeting at the vertices and further connected by thin blue lines, then we should distinguish between five cases:

(a) merged are two vertices inside a thick propagator

(b) merged are two vertices in two thick propagators of the same color

(c) merged are two vertices in two thick propagators of two different colors

(d) merged are two inter-propagator vertices

(e) merged are the inter-propagator vertex and that inside a thick propagator.

It is easy to see that

(a) leads to decoupling of a closed piece of the thick line, i.e. of the average $\left\langle\mathcal{K}_{m_{2}}\right\rangle$

(b) leads to overcrossing of the two propagators

(c) leads to emerging of two new inter-propagator vertices connected by a blue line

(d) connects the two remote vertices and releases two thick propagators

(e) exchanges a piece of the thick propagator 
Pictorially:

(a)

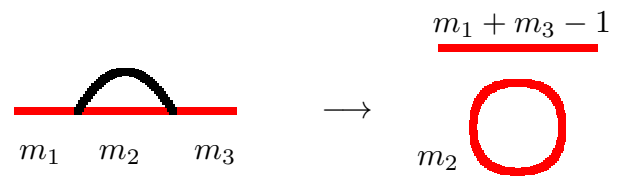

$$
\left(\operatorname{Tr} \frac{\partial}{\partial A} \frac{\partial}{\partial A}\right)\left[\ldots A_{i}^{p a} \bar{A}_{p a}^{k} \hat{O}_{k}^{j} A_{j}^{q b} \bar{A}_{q b}^{l} \ldots\right]=\left[\ldots A_{i}^{p a} \hat{O}_{k}^{k} \bar{A}_{p a}^{l} \ldots\right]
$$

(b)

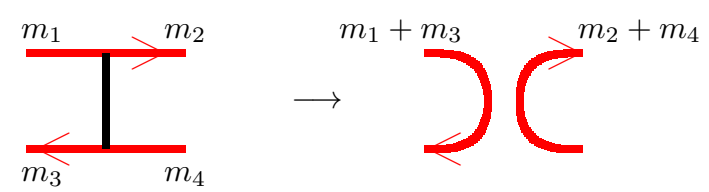

$\left(\operatorname{Tr} \frac{\partial}{\partial A} \frac{\partial}{\partial A}\right)\left[\ldots A_{i}^{p a} \bar{A}_{p a}^{k} \hat{O}_{k}^{j}\right]\left[\hat{O}_{n}^{\prime m} A_{m}^{q b} \bar{A}_{q b}^{l} \ldots\right]=\left[\ldots A_{i}^{p a} \hat{O}_{k}^{j}\right]\left[\hat{O}_{n}^{\prime k} \bar{A}_{p a}^{l} \ldots\right]=\left[\ldots A_{i}^{p a} \bar{A}_{p a}^{l} \ldots\right]\left[\left(\hat{O} \hat{O}^{\prime}\right)_{n}^{j}\right]$

(c)

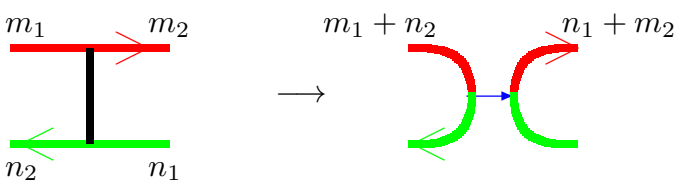

$\left(\operatorname{Tr} \frac{\partial}{\partial A} \frac{\partial}{\partial A}\right)\left[\ldots A_{i}^{p a} \bar{A}_{q a}^{i} \hat{O}_{r}^{q}\right]\left[\hat{O}_{k}^{\prime j} A_{j}^{s b} \bar{A}_{s b}^{l} \ldots\right]=\left[\ldots A_{i}^{p a} \hat{O}_{r}^{q}\right]\left[\hat{O}_{k}^{\prime i} \bar{A}_{q a}^{l} \ldots\right]=\left[\ldots A_{i}^{p a} \hat{O}_{k}^{\prime i}\right]\left[\hat{O}_{r}^{q} \bar{A}_{q a}^{l} \ldots\right]$

$(d)$

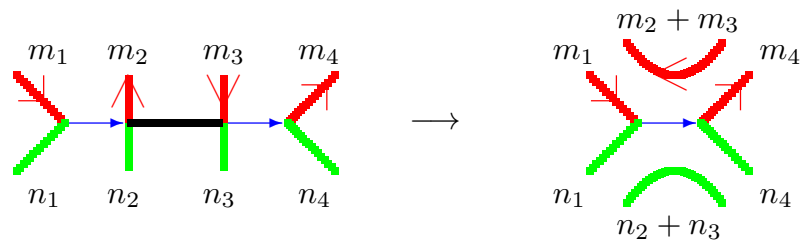

$\left(\operatorname{Tr} \frac{\partial}{\partial A} \frac{\partial}{\partial A}\right)\left[\ldots A_{i}^{p a} \hat{O}_{k}^{i}\right]\left[\hat{O}_{r}^{\prime q} \bar{A}_{q a}^{l} \ldots\right]\left[\ldots A_{j}^{s b} \hat{O}_{m}^{j}\right]\left[{\hat{\hat{O}_{t}^{\prime u}}}_{t}^{\prime n} \bar{A}_{u b}^{n} \ldots\right]=\left[\ldots A_{i}^{p a} \hat{O}_{k}^{i}\right]\left[\hat{\bar{O}}_{t}^{\prime q} \bar{A}_{q a}^{n} \ldots\right]\left[\hat{O}_{r}^{\prime s} \ldots\right]\left[\ldots \hat{O}_{m}^{l}\right]$

$(e)$

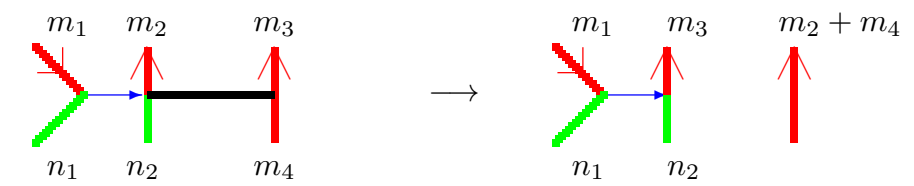

$$
\left(\operatorname{Tr} \frac{\partial}{\partial A} \frac{\partial}{\partial A}\right)\left[\ldots A_{i}^{p a} \hat{O}_{k}^{i}\right]\left[\ldots \bar{A}_{q a}^{o} \hat{O}_{o}^{\prime l}\right]\left[\ldots A_{m}^{s b} \bar{A}_{s b}^{j} \hat{O}_{j}^{\prime \prime n}\right]=\left[\ldots A_{i}^{p a} \hat{O}_{k}^{i}\right]\left[\ldots \hat{O}_{m}^{\prime l}\right]\left[\ldots \bar{A}_{q a}^{j} \hat{O}_{j}^{\prime \prime n}\right]
$$

In result, we obtain that a closed set of operators is formed by tri-valent vertices connected by thin blue lines and thick red and green lines, which carry additional "length" labels. It is now clear that the Laplace operator at the r.h.s. of the recursion relation (5.2) does not take us away from this restricted set of operators, i.e. we get a closed set of Ward identities. This makes such models potentially solvable, though there is still a long way 
to go before these solutions are made as clear and explicit as in [16-24] for the ordinary matrix models.

\subsection{The structure of RG-complete set of operators}

The previous subsection provides a description of the theory (5.1) in terms of the decorated tri-valent graphs.

The keystone operators $\mathcal{K}_{2}$ and $\mathcal{K}_{2}$ are now depicted as red and green circles of length 2.

The tree operators are chains of these operators connected by thick black lines, which can be then eliminated by the rules (a)-(e). The same is true about the loop operators.

The trees made from $\mathcal{K}_{2}$ alone are fully handled by rule (b), and they are just red circles of arbitrary length $m$. The loops made from $\mathcal{K}_{2}$ are also handled by rule (a) and they are disconnected collections of red circles of arbitrary lengths. This simple structure of the RG-completion of $\mathcal{K}_{2}$ was actually underlying the solution of the model in section 4 :

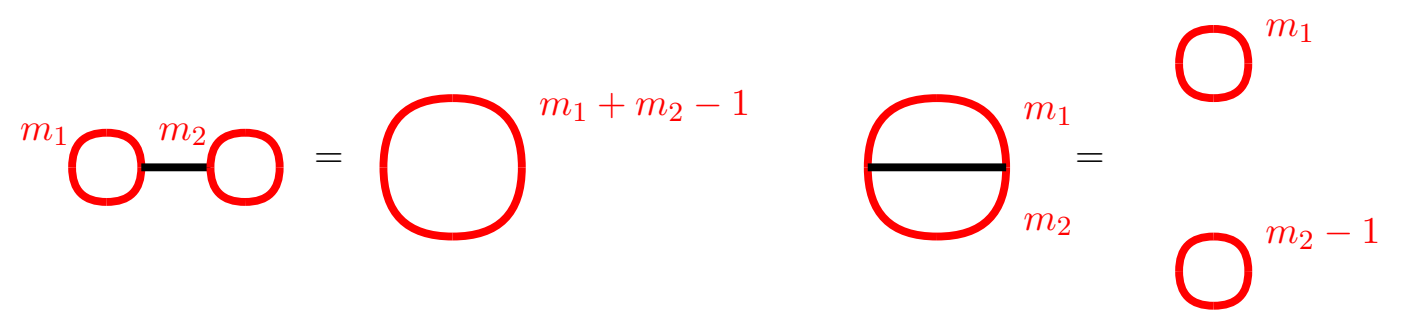

The same is true for the RG-completion of $\mathcal{K}_{2}$ :

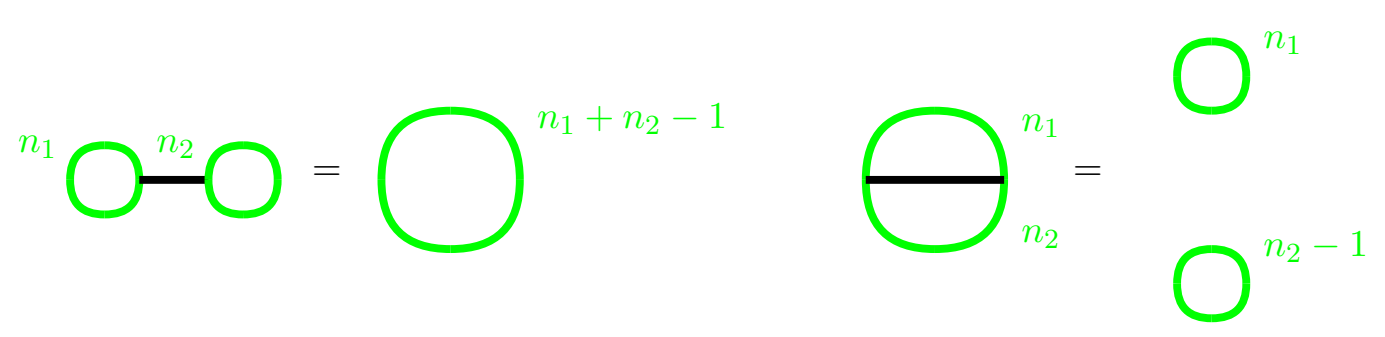

Something new arises when the tree operators involve chains with both types of the Feynman diagram vertices $\mathcal{K}_{2}$ and $\mathcal{K}_{2}$. Now rule (c) is needed and emerging are the operator $\mathcal{K}_{m, n}$ and, further, arbitrary red-green cycles with non-intersecting thin blue shortcuts. Thus all tree-operators are single planar cycles:

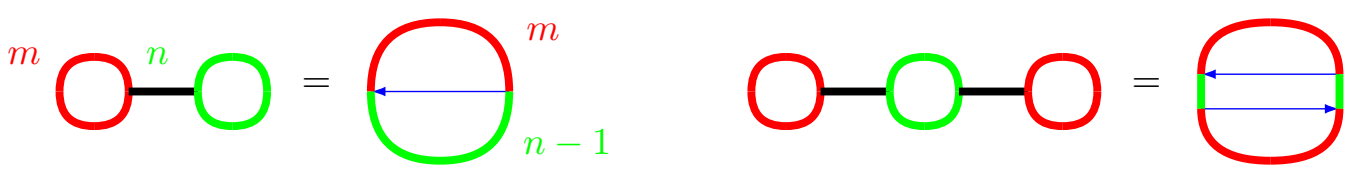

The loop operators are either the red-green cycles with the intersecting blue shortcuts or several such red-green cycles with the shortcuts connected by thin blue lines.
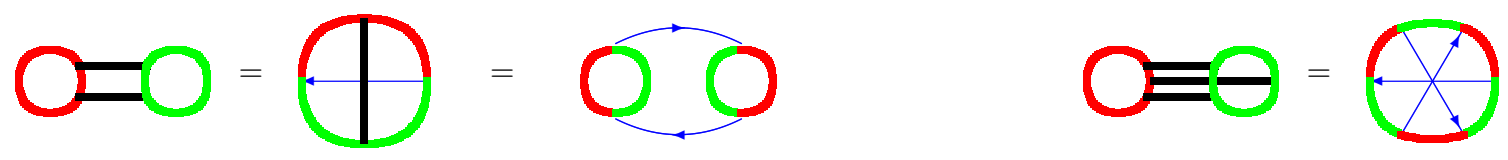
This describes the set of operators in the extended action of the RG-completed model (5.1). Clearly, they have an interpretation in terms of some quantum mechanics: a one-dimensional QFT defined on a collection of circles (which, in turn, can be thought of as boundaries of holes on a plane). The circles are equipped with lengths of their segments (thick red and green propagators), and the field in this new effective $1 \mathrm{~d}$ theory is responsible for the thin blue lines connecting arbitrary points of arbitrary circles. Since the relevant lengths are integer, this quantum mechanics should be discrete and, perhaps, $p$-adic. Following this line one can also approach the old problem of relating the BZ forest formulas with the Bruhat-Tits trees, i.e. finding a $p$-adic interpretation of the measures on the space of trees which the BZ theory associates with arbitrary QFT with a chosen keystone operator. This can bring us back to the old attempts of $[182,183]$ in the simplest string models, to their reformulation in terms of matrix models and further generalizations to tensor models.

\subsection{Towards Ward identities}

The recursion relations (5.2), i.e. the equations of motion in the theory (5.1), are now the defining relations (equivalencies) between the contributions of decorated tri-valent graphs.

The simplest recursion relation we already encountered in section 2.1.3, it remains just the same:

or, pictorially,

$$
\mu\left\langle\mathcal{K}_{m}\right\rangle=\sum_{m_{1}+m_{2}=m-1}=\left\langle\mathcal{K}_{m_{1}} \mathcal{K}_{m_{2}}\right\rangle
$$

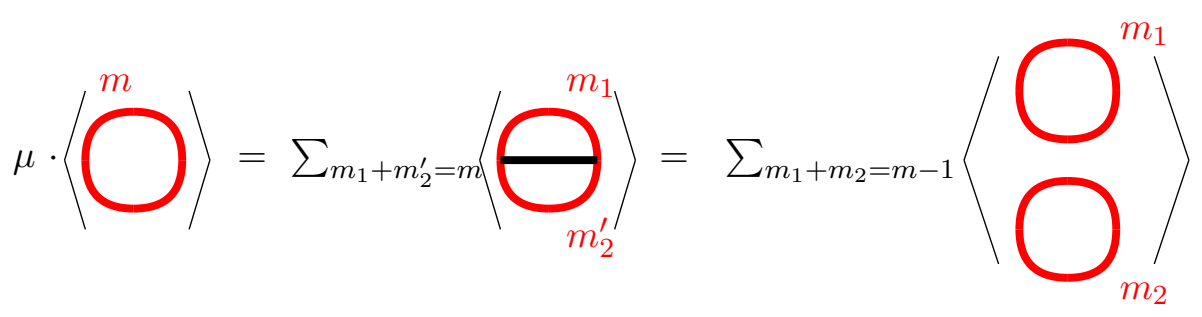

A similar relation holds in the green sector.

The first recursion which mixes the red and green colorings is

$$
\begin{aligned}
\mu \cdot(m+n) \cdot\left\langle\mathcal{K}_{m} \mathcal{K}_{n}\right\rangle= & m \cdot \sum_{m_{1}+m_{2}=m-1}\left\langle\mathcal{K}_{m_{1}} \mathcal{K}_{m_{2}} \mathcal{K}_{n}\right\rangle \\
& +n \cdot \sum_{n_{1}+n_{2}=n-1}\left\langle\mathcal{K}_{m} \mathcal{K}_{n_{1}} \mathcal{K}_{n_{2}}\right\rangle+2 m n \cdot\left\langle\mathcal{K}_{m, n}\right\rangle
\end{aligned}
$$

Pictorially, with coefficients and summations omitted,

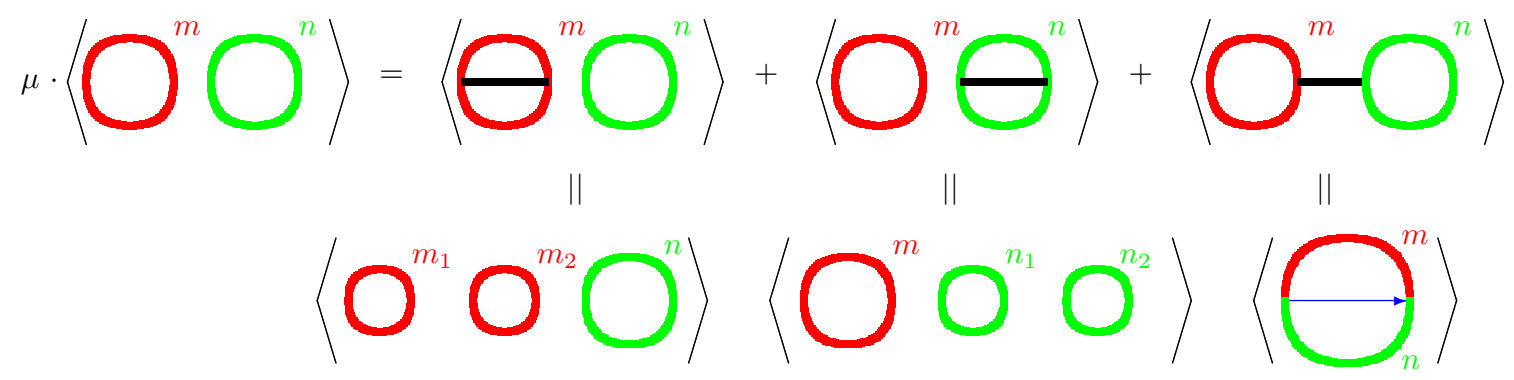


Each correlator at the both sides of such recursions is by itself a result of calculations with the help of the Wick theorem. The recursion describes insertion of just a single propagator: it connect the Feynman diagram with "smaller" Feynman diagrams, which contain less background fields (by two) and smaller power of $\mu^{-1}$ (by one). Instead, it converts the tree operators into the loop ones, i.e. increases the number of thick colored circles. In the above examples, they are disconnected, but in general thin blue lines will appear between them, e.g.

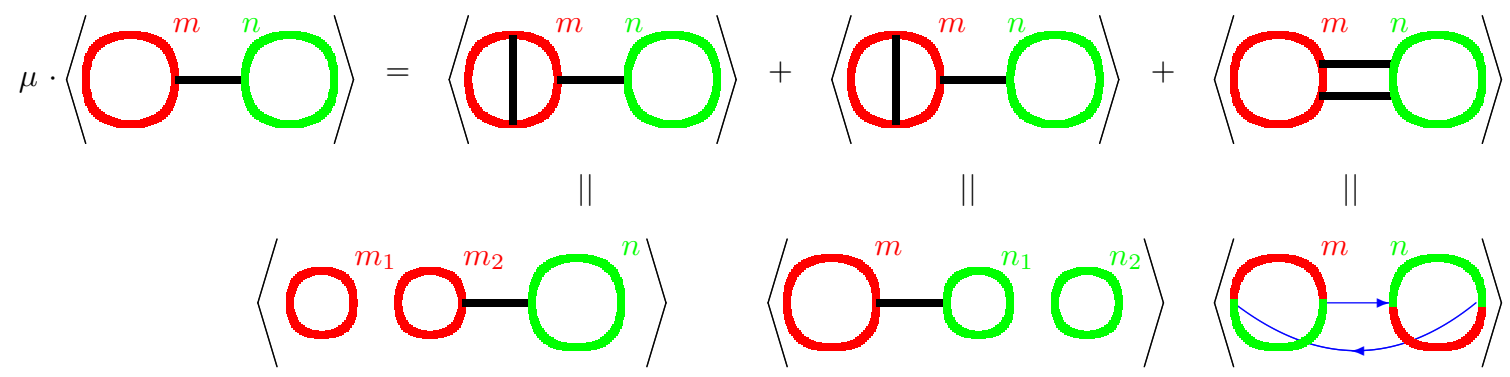

(combinatorial coefficients can be easily restored).

These are the simplest examples of recursions in the simplest non-trivial tensor model (5.1). The next problems to look at will be:

(1) To calculate particular averages as it was done in section 5.3 and to check that they satisfy these recursions. Already this is a rather tedious exercise.

(1a) As an important deviation, one can look at particular large- $N$ limits, where the averages simplify. What needs to be found there is an analogue of the factorization properties, allowing one to simplify disconnected correlators and those with the intersecting blue lines. This is also a line leading to a description in terms of the spectral curves and the AMM/EO topological recursion. The subsequent steps below can also be done separately for generic $N$ and in the limit, and then lifted back to generic $N$ with the help of the genus expansion (which will be not literally genus beyond matrix models).

(1b) Alternatively, one can put one of $N$ 's equal to one and study the emerging (not quite trivial) reduction to the complex matrix model, or, what is the same, to the "red" quasi-tensor model in section 4 . This is a simpler, still an exciting exercise, and this model has its own large- $N$ limits, AMM/EO-topological recursions, check operators etc, all being under-investigated (see, however, [184] for some models). What facilitates this particular study are the known Virasoro constraints (4.4) and their amusing indirect corollary (4.14), which drastically simplifies the examination of the recursion when some circle are of unit length.

(2) To find the analogue of general formulas like (2.21) or (2.27) and check that the simple recursions of this subsection are satisfied functorially in the parameters $m, n$ and promote them to particular generating functions. 
(3) To find generating function(al) of another level, describing the entire set of recursion relations, of which the ones mentioned in this subsection are just the simplest examples.

(4) To proceed to a more complicated rainbow and then to the uncolored tensor models.

\subsection{Towards solvability of tensor models}

Actually, in our consideration of matrix models in section 2 and section 4, we discovered that the infinite Virasoro recursion is not the maximal structure one can look for: there are also finite and linear relations (2.27) between Gaussian correlators. The reason for their existence is presumably the interplay, a combination of infinite linear Virasoro constraints and of the quadratic Hirota relations that reflect integrability. The matrix model $\tau$-functions are peculiar objects in the intersection of these two worlds, and now we recognized that, as was long expected, this indeed leads to a full solvability.

It is natural to look in the same direction in analysis of the tensor models. In this paper, we discussed in some detail what the recursion means in this case. What substitutes integrability for the tensor models is still a mystery. However, we can attempt to bypass this problem, and look directly at finite relations between correlators. A part of the problem is that the relations like (2.27) are not homogeneous: the correlators are expressed through some more fundamental objects, dimensions, i.e. the values characters at the topological locus [164-168]. However, it is not a priori clear what should play their role in the tensor case. We postpone a detailed discussion on this subject in order to avoid mixing clear facts reported in the present paper, with speculations and fantasies. Here me provide just a very simple evidence that things can work.

The averages $\left\langle\mathcal{K}_{\Lambda}\right\rangle$ are already expressed through quantities like $D_{R}\left(N_{r}\right)$ and $D_{R}\left(N_{g} N_{b}\right)$. A more accurate characteristic of emerging quantities is that their double Fourier transforms factorize, see eq. (2.56) and discussion after it. The same is true for expressing $<\mathcal{K}_{\Lambda}>$ through the quantities from the class of $D_{R}\left(N_{g}\right)$ and $D_{R}\left(N_{r} N_{b}\right)$, and, actually, for their blue analogues $\left\langle\mathcal{K}_{\Lambda}>\right.$ through $D_{R}\left(N_{b}\right)$ and $D_{R}\left(N_{r} N_{g}\right)$. Thus, the question is about the new, essentially tensor model correlators, which were not present in matrix models. At the simple level, which we are at in the present paper, we should look at least for expressions of the first non-trivial correlators in the Aristotelian model, $\left\langle\mathcal{K}_{m} \mathcal{K}_{n}\right\rangle$ and $\left\langle\mathcal{K}_{m, n}\right\rangle$ through $\left\langle\mathcal{K}_{m}\right\rangle$ and $\left\langle\mathcal{K}_{n}\right\rangle$, i.e. through the variables $\alpha$ and $\beta$. Surprisingly or not, such expressions indeed exist: formulas from section 5.3 can be converted into

$$
\begin{aligned}
& \beta \cdot\left\langle K_{2,2}\right\rangle=\beta^{2} \cdot\left(\alpha_{r} \alpha_{g}+\alpha_{b}\right)=\left\langle\mathcal{K}_{2}\right\rangle\left\langle\mathcal{K}_{2}\right\rangle+\left\langle\mathcal{K}_{1}\right\rangle\left\langle\mathcal{K}_{2}\right\rangle \\
& \beta \cdot\left\langle K_{3,2}\right\rangle=\beta^{2} \cdot\left(\left(\alpha_{r}^{2}+\beta+1\right) \cdot \alpha_{g}+2 \cdot\left(\alpha_{r} \alpha_{b}+\alpha_{g}\right)\right)=\left\langle\mathcal{K}_{3}\right\rangle\left\langle\mathcal{K}_{2}\right\rangle+2 \cdot \beta^{2} \cdot\left(\alpha_{r} \alpha_{b}+\alpha_{g}\right) \\
& \beta \cdot\left\langle K_{4,2}\right\rangle=\beta^{2} \cdot\left(\alpha_{r}\left(\alpha_{r}^{2}+3 \beta+5\right) \cdot \alpha_{g}+\left(3 \alpha_{r}^{2}+2 \beta+1\right) \alpha_{b}+7 \cdot\left(\alpha_{r} \alpha_{g}+\alpha_{b}\right)\right)=\left\langle\mathcal{K}_{4}\right\rangle\left\langle\mathcal{K}_{2}\right\rangle+\ldots
\end{aligned}
$$


and

$$
\begin{aligned}
\beta \cdot\left\langle\mathcal{K}_{2} \mathcal{K}_{2}\right\rangle & =\beta^{2} \cdot\left(\alpha_{r} \alpha_{g} \cdot(\beta+4)+2 \cdot \alpha_{b}\right)=\left\langle\mathcal{K}_{2}\right\rangle\left\langle\mathcal{K}_{2}\right\rangle \cdot(2 \cdot 2+\beta)+2 \cdot\left\langle\mathcal{K}_{1}\right\rangle\left\langle\mathcal{K}_{2}\right\rangle \\
\beta \cdot\left\langle\mathcal{K}_{3} \mathcal{K}_{2}\right\rangle & =\beta^{2} \cdot\left(\left(\alpha_{r}^{2}+\beta+1\right) \cdot \alpha_{g} \cdot(\beta+6)+6 \cdot\left(\alpha_{r} \alpha_{b}+\alpha_{g}\right)\right) \\
& =\left\langle\mathcal{K}_{3}\right\rangle\left\langle\mathcal{K}_{2}\right\rangle \cdot(3 \cdot 2+\beta)+6 \beta^{2} \cdot\left(\alpha_{r} \alpha_{b}+\alpha_{g}\right) \\
\beta \cdot\left\langle\mathcal{K}_{4} \mathcal{K}_{2}\right\rangle & =\beta^{2} \cdot\left(\alpha_{r}\left(\alpha_{r}^{2}+3 \beta+5\right) \cdot \alpha_{g} \cdot(\beta+8)+4 \cdot\left(3 \alpha_{r}^{2}+2 \beta+1\right) \cdot \alpha_{b}+28 \cdot\left(\alpha_{r} \alpha_{g}+\alpha_{b}\right)\right)= \\
& =\left\langle\mathcal{K}_{4}\right\rangle\left\langle\mathcal{K}_{2}\right\rangle \cdot(4 \cdot 2+\beta)+\ldots
\end{aligned}
$$

Moreover, some combinations familiar from the table (4.9), show up in these expressions. The two next relations include $\left\langle K_{3 W}\right\rangle$ from (5.34), which, at the tensor model level, should probably be included into the set of dimension-like objects:

$$
\begin{aligned}
\beta \cdot\left\langle\mathcal{K}_{3,3}\right\rangle= & \beta^{2} \cdot\left(\left(\alpha_{r}^{2}+\beta+1\right) \cdot\left(\alpha_{g}^{2}+\beta+1\right)+4 \cdot\left(\alpha_{r}^{2}+\beta+1\right)+\left(\alpha_{b}^{2}+\beta+1\right)+4 \cdot\left(\alpha_{r} \alpha_{b}+\alpha_{g}\right) \cdot \alpha_{g}\right)+ \\
& +\beta \cdot\left\langle K_{3 W}\right\rangle=\left\langle\mathcal{K}_{3}\right\rangle\left\langle\mathcal{K}_{3}\right\rangle+4 \cdot\left\langle\mathcal{K}_{1}\right\rangle\left\langle\mathcal{K}_{3}\right\rangle+\left\langle\mathcal{K}_{1}\right\rangle\left\langle\mathcal{K}_{3}\right\rangle+\ldots+\left\langle K_{1}\right\rangle\left\langle K_{3 W}\right\rangle \\
\beta \cdot\left\langle\mathcal{K}_{4,3}\right\rangle= & \beta^{2} \cdot\left(\alpha_{r}\left(\alpha_{r}^{2}+3 \beta+5\right) \cdot\left(\alpha_{g}^{2}+\beta+1\right)+6 \cdot \alpha_{r}\left(\alpha_{r}^{2}+3 \beta+5\right)\right. \\
& +3 \cdot \alpha_{r} \cdot\left(\alpha_{b}^{2}+\beta+1\right)+2 \cdot\left(3 \alpha_{r}^{2}+2 \beta+1\right) \alpha_{b} \cdot \alpha_{g}+ \\
& \left.+14\left(\alpha_{r} \alpha_{g}+\alpha_{b}\right) \cdot \alpha_{g}+12\left(\alpha_{g} \alpha_{b}+\alpha_{r}\right)\right)+3 \alpha_{r} \beta \cdot\left\langle K_{3 W}\right\rangle= \\
= & \left\langle\mathcal{K}_{4}\right\rangle\left\langle\mathcal{K}_{3}\right\rangle+6 \cdot\left\langle\mathcal{K}_{1}\right\rangle\left\langle\mathcal{K}_{4}\right\rangle+3 \cdot\left\langle\mathcal{K}_{2}\right\rangle\left\langle\mathcal{K}_{3}\right\rangle+\ldots+3 \cdot\left\langle K_{2}\right\rangle\left\langle K_{3 W}\right\rangle
\end{aligned}
$$

and

$$
\begin{aligned}
\beta \cdot\left\langle\mathcal{K}_{3} \mathcal{K}_{3}\right\rangle= & \beta^{2} \cdot\left(\left(\alpha_{r}^{2}+\beta+1\right) \cdot\left(\alpha_{g}^{2}+\beta+1\right) \cdot(\beta+9)+18 \cdot\left(\alpha_{r}^{2}+\beta+1\right)+\right. \\
& \left.+3 \cdot\left(\alpha_{b}^{2}+\beta+1\right)+18 \cdot\left(\alpha_{r} \alpha_{b}+\alpha_{g}\right) \cdot \alpha_{g}\right)+3 \beta \cdot\left\langle K_{3 W}\right\rangle \\
= & \left\langle\mathcal{K}_{3}\right\rangle\left\langle\mathcal{K}_{3}\right\rangle \cdot(3 \cdot 3+\beta)+18 \cdot\left\langle\mathcal{K}_{1}\right\rangle\left\langle\mathcal{K}_{3}\right\rangle+ \\
& +3 \cdot\left\langle\mathcal{K}_{1}\right\rangle\left\langle\mathcal{K}_{3}\right\rangle+\ldots+3 \cdot\left\langle K_{1}\right\rangle\left\langle K_{3 W}\right\rangle
\end{aligned}
$$

We omitted $\mu$ factors to avoid overloading the formulas.

Is this exactly what we could dream about?

Not quite: there are four not quite expected new features.

First, not only $\alpha_{r}=N_{r}+N_{g} N_{b}$ and $\alpha_{g}=N_{g}+N_{r} N_{b}$ appear in these expressions, but also $\alpha_{b}=N_{b}+N_{r} N_{g}$. We remind that $\beta=N_{r} N_{g} N_{b}$ is symmetric in the three colorings. The four quantities $\alpha_{r}, \alpha_{g}, \alpha_{b}$ and $\beta$ are, of course, not independent, but relation between them is irrational. More than that, additional quantities like $\left\langle\mathcal{K}_{3 W}\right\rangle$ can need to be added to the set of "dimensions". 
Second, new peculiar combinations emerge involving the blue $\alpha_{b}$, which are not immediately seen in (4.9). They are explicitly written in the intermediate formulas and are substituted by dots at the r.h.s. Presumably, they are made from correlators which mix the green and blue operators and were not calculated in section 5.3.

Third, grading is not fully respected. Still, these formulas are quite different from the Virasoro-related recursions like (5.46) and have a potential to reach the capacity of (2.27) after more examples are worked out and the structure is fully revealed.

Fourth, expressions look at best quadratic in correlators from (4.9). To see this better, one can interpret the additional $\beta$ factors at the l.h.s. as $\left\langle\mathcal{K}_{1}\right\rangle=\beta$.

Thus, the relations we are searching for seem to respect the symmetry between the three colors (what is natural to expect from a fundamental property of the model), but they can actually be non-linear. Since in the matrix models these relations were linear and there they substituted the quadratic Hirota equations, this can mean that the substitute of integrable structure in the tensor case is going to be not quadratic, but have a higher degree of non-linearity, perhaps, in a spirit of the generalized Nambu structure.

All this opens a new exciting perspective for further development of the tensor models.

\section{Conclusion}

In this paper, we reviewed the general notion of RG-completeness and the BZ-induced theory of the universal Virasoro-like constraints (with the Virasoro algebra substituted by that of the rooted trees) in application to matrix and tensor models. A relatively detailed presentation was given of a simple Aristotelian ("red-green" or RGB) tensor model (5.1), with explicit examples of the Gaussian averages and relations between them, some related to the Ward identities, some to a still hidden integrability-like structure. This illustrates a possibility of identifying the RG-completions of the tensor models as potentially solvable, perhaps to the extent of solvability of the simplest matrix models, which we also raised in this paper to a qualitatively new level. However, this solvability needs still to be studied and much more remains to be done in the case of more interesting rainbow models with tetrahedron-like vertices. Fortunately, referring to Aristotle's celebrated thesis that "...three completes the series of colours (as we find three does in most other things), and the change into the rest is imperceptible to sense ..." [177], we can hope that the study of RGB model advanced in the present paper rightly captures the most important sides of the story.

\section{Acknowledgments}

The work of H.I. was supported in part by JSPS KAKENHI Grant Number 15K05059. A.Mor. acknowledges the support of JSPS (\# S16124) and hospitality of OCU. Our work was partly supported by RFBR grants 16-01-00291 (A.Mir.), 16-02-01021 (A.Mor.) and by joint grants 17-51-50051-YaF, 15-51-52031-NSC-a, 16-51-53034-GFEN, 16-51-45029-INDa. Support from JSPS/RFBR bilateral collaborations "Faces of matrix models in quantum field theory and statistical mechanics" is gratefully appreciated. 
Open Access. This article is distributed under the terms of the Creative Commons Attribution License (CC-BY 4.0), which permits any use, distribution and reproduction in any medium, provided the original author(s) and source are credited.

\section{References}

[1] N. Bogolubov and O. Parasyuk, On the Multiplication of the causal function in the quantum theory of fields, Acta Math. 97 (1957) 227 [INSPIRE].

[2] N. Bogolubov and D. Shirkov, Introduction to the Theory of Quantum Fields1, Moscow (1957).

[3] B. Stepanov and O. Zavyalov, Asymptotics of divergent Feynman diagrams, Yad. Phys. 1 (1965) 922.

[4] K. Hepp, Proof of the Bogolyubov-Parasiuk theorem on renormalization, Commun. Math. Phys. 2 (1966) 301 [INSPIRE].

[5] W. Zimmermann, Convergence of Bogolyubov's method of renormalization in momentum space, Commun. Math. Phys. 15 (1969) 208 [INSPIRE].

[6] F. David, Loop Equations and Nonperturbative Effects in Two-dimensional Quantum Gravity, Mod. Phys. Lett. A 5 (1990) 1019 [InSPIRE].

[7] A. Mironov and A. Morozov, On the origin of Virasoro constraints in matrix models: Lagrangian approach, Phys. Lett. B 252 (1990) 47 [INSPIRE].

[8] J. Ambjørn and Yu.M. Makeenko, Properties of Loop Equations for the Hermitean Matrix Model and for Two-dimensional Quantum Gravity, Mod. Phys. Lett. A 5 (1990) 1753 [INSPIRE].

[9] H. Itoyama and Y. Matsuo, Noncritical Virasoro algebra of $d<1$ matrix model and quantized string field, Phys. Lett. B 255 (1991) 202 [INSPIRE].

[10] H. Itoyama and Y. Matsuo, $W_{1+\infty}$ type constraints in matrix models at finite $N$, Phys. Lett. B 262 (1991) 233 [INSPIRE].

[11] A.S. Alexandrov, A. Mironov and A. Morozov, Unified description of correlators in non-Gaussian phases of Hermitean matrix model, Int. J. Mod. Phys. A 21 (2006) 2481 [hep-th/0412099] [INSPIRE].

[12] A.S. Alexandrov, A. Mironov and A. Morozov, Solving Virasoro constraints in matrix models, Fortsch. Phys. 53 (2005) 512 [hep-th/0412205] [INSPIRE].

[13] A. Mironov and A. Morozov, Check-operators and Quantum Spectral Curves, arXiv: 1701.03057 [INSPIRE].

[14] D. Galakhov, A. Mironov and A. Morozov, S-duality and Modular Transformation as a non-perturbative deformation of the ordinary pq-duality, JHEP 06 (2014) 050 [arXiv:1311.7069] [INSPIRE].

[15] D. Galakhov, A. Mironov and A. Morozov, Wall Crossing Invariants: from quantum mechanics to knots, J. Exp. Theor. Phys. 120 (2015) 549 [arXiv:1410.8482] [InSPIRE].

[16] A. Morozov, String theory: what is it?, Phys. Usp. (UFN) 35 (1992) 671.

[17] A. Morozov, Integrability and matrix models, Phys. Usp. (UFN) 37 (1994) 1.

[18] A. Morozov, Matrix models as integrable systems, hep-th/9502091 [INSPIRE]. 
[19] A. Morozov, Challenges of matrix models, hep-th/0502010 [INSPIRE].

[20] A. Mironov, 2-D gravity and matrix models. 1. 2-D gravity, Int. J. Mod. Phys. A 9 (1994) 4355 [hep-th/9312212] [INSPIRE].

[21] A. Mironov, Matrix models of two-dimensional gravity, Phys. Part. Nucl. 33 (2002) 537 [INSPIRE].

[22] A. Mironov, Quantum deformations of tau functions, bilinear identities and representation theory, hep-th/9409190 [INSPIRE].

[23] H. Itoyama and R. Yoshioka, Developments of theory of effective prepotential from extended Seiberg-Witten system and matrix models, PTEP 2015 (2015) 11B103 [arXiv:1507.00260] [INSPIRE].

[24] H. Itoyama, Developments of Supersymmetric Gauge Theory by Matrices (in Japanese), Butsuri 71 (2016) 607.

[25] H. Itoyama, A. Mironov and A. Morozov, Rainbow tensor model with enhanced symmetry and extreme melonic dominance, Phys. Lett. B 771 (2017) 180 [arXiv:1703.04983] [INSPIRE].

[26] R.C. Myers and V. Periwal, From polymers to quantum gravity: Triple scaling in rectangular random matrix models, Nucl. Phys. B 390 (1993) 716 [hep-th/9112037] [INSPIRE].

[27] R. Lafrance and R.C. Myers, Flows for rectangular matrix models, Mod. Phys. Lett. A 9 (1994) 101 [hep-th/9308113] [INSPIRE].

[28] P. Di Francesco, Rectangular matrix models and combinatorics of colored graphs, Nucl. Phys. B 648 (2003) 461 [cond-mat/0208037] [INSPIRE].

[29] E. Witten, An SYK-Like Model Without Disorder, arXiv:1610.09758 [InSPIRE].

[30] R. Gurau, The complete $1 / N$ expansion of a SYK-like tensor model, Nucl. Phys. B 916 (2017) 386 [arXiv: 1611.04032] [INSPIRE].

[31] R. Gurau, Quenched equals annealed at leading order in the colored SYK model, arXiv: 1702.04228 [INSPIRE].

[32] I.R. Klebanov and G. Tarnopolsky, Uncolored random tensors, melon diagrams and the Sachdev-Ye-Kitaev models, Phys. Rev. D 95 (2017) 046004 [arXiv:1611.08915] [InSPIRE].

[33] S. Carrozza and A. Tanasa, $O(N)$ Random Tensor Models, Lett. Math. Phys. 106 (2016) 1531 [arXiv: 1512.06718] [INSPIRE].

[34] D.J. Gross and V. Rosenhaus, A Generalization of Sachdev-Ye-Kitaev, JHEP 02 (2017) 093 [arXiv: 1610.01569$]$ [INSPIRE].

[35] D.J. Gross and V. Rosenhaus, The Bulk Dual of SYK: Cubic Couplings, JHEP 05 (2017) 092 [arXiv: 1702.08016] [INSPIRE].

[36] C. Krishnan, S. Sanyal and P.N. Bala Subramanian, Quantum Chaos and Holographic Tensor Models, JHEP 03 (2017) 056 [arXiv:1612.06330] [INSPIRE].

[37] F. Ferrari, The Large D Limit of Planar Diagrams, arXiv:1701.01171 [INSPIRE].

[38] V. Bonzom, L. Lionni and A. Tanasa, Diagrammatics of a colored SYK model and of an SYK-like tensor model, leading and next-to-leading orders, J. Math. Phys. 58 (2017) 052301 [arXiv: 1702.06944] [INSPIRE]. 
[39] M. Beccaria and A.A. Tseytlin, Partition function of free conformal fields in 3-plet representation, JHEP 05 (2017) 053 [arXiv: 1703.04460] [INSPIRE].

[40] S. Sachdev and J. Ye, Gapless spin fluid ground state in a random, quantum Heisenberg magnet, Phys. Rev. Lett. 70 (1993) 3339 [cond-mat/9212030] [INSPIRE].

[41] J. Polchinski and V. Rosenhaus, The spectrum in the Sachdev-Ye-Kitaev Model, JHEP 04 (2016) 001 [arXiv: 1601.06768] [inSPIRE].

[42] W. Fu, D. Gaiotto, J. Maldacena and S. Sachdev, Supersymmetric Sachdev-Ye-Kitaev models, Phys. Rev. D 95 (2017) 026009 [arXiv:1610.08917] [InSPIRE].

[43] M. Berkooz, P. Narayan, M. Rozali and J. Simón, Higher Dimensional Generalizations of the SYK Model, JHEP 01 (2017) 138 [arXiv: 1610.02422] [INSPIRE].

[44] A. Kitaev, A simple model of quantum holography, talks at KITP, 7 April and 27 May 2015 [http://online.kitp.ucsb.edu/online/entangled15/kitaev/] [http://online.kitp.ucsb.edu/online/entangled15/kitaev2/].

[45] S. Sachdev, Bekenstein-Hawking Entropy and Strange Metals, Phys. Rev. X 5 (2015) 041025 [arXiv: 1506.05111] [INSPIRE].

[46] A. Jevicki, K. Suzuki and J. Yoon, Bi-Local Holography in the SYK Model, JHEP 07 (2016) 007 [arXiv: 1603.06246] [INSPIRE].

[47] J. Maldacena and D. Stanford, Remarks on the Sachdev-Ye-Kitaev model, Phys. Rev. D 94 (2016) 106002 [arXiv: 1604.07818] [INSPIRE].

[48] D. Bagrets, A. Altland and A. Kamenev, Sachdev-Ye-Kitaev model as Liouville quantum mechanics, Nucl. Phys. B 911 (2016) 191 [arXiv:1607.00694] [INSPIRE].

[49] A. Jevicki and K. Suzuki, Bi-Local Holography in the SYK Model: Perturbations, JHEP 11 (2016) 046 [arXiv: 1608.07567] [INSPIRE].

[50] Z. Bi, C.-M. Jian, Y.-Z. You, K.A. Pawlak and C. Xu, Instability of the non-Fermi liquid state of the Sachdev-Ye-Kitaev Model, Phys. Rev. B 95 (2017) 205105 [arXiv:1701.07081] [INSPIRE].

[51] S.-K. Jian and H. Yao, Solvable SYK models in higher dimensions: a new type of many-body localization transition, arXiv: 1703.02051 [INSPIRE].

[52] S. Carrozza, V. Lahoche and D. Oriti, Renormalizable Group Field Theory beyond melons: an example in rank four, arXiv:1703.06729 [INSPIRE].

[53] C. Krishnan, K.V.P. Kumar and S. Sanyal, Random Matrices and Holographic Tensor Models, arXiv:1703.08155 [INSPIRE].

[54] M.R. Casali, P. Cristofori, S. Dartois and L. Grasselli, Topology in colored tensor models via crystallization theory, arXiv:1704.02800 [INSPIRE].

[55] C. Peng, Vector models and generalized SYK models, JHEP 05 (2017) 129 [arXiv: 1704.04223] [INSPIRE].

[56] S.R. Das, A. Jevicki and K. Suzuki, Three Dimensional View of the SYK/AdS Duality, arXiv: 1704.07208 [INSPIRE].

[57] H. Kyono, S. Okumura and K. Yoshida, Comments on $2 D$ dilaton gravity system with a hyperbolic dilaton potential, arXiv:1704.07410 [INSPIRE]. 
[58] F. David, Planar Diagrams, Two-Dimensional Lattice Gravity and Surface Models, Nucl. Phys. B 257 (1985) 45 [inSPIRE].

[59] V.A. Kazakov, A.A. Migdal and I.K. Kostov, Critical Properties of Randomly Triangulated Planar Random Surfaces, Phys. Lett. B 157 (1985) 295 [inSPIRE].

[60] J. Ambjørn, B. Durhuus and T. Jonsson, Three-dimensional simplicial quantum gravity and generalized matrix models, Mod. Phys. Lett. A 6 (1991) 1133 [InSPIRE].

[61] N. Sasakura, Tensor model for gravity and orientability of manifold, Mod. Phys. Lett. A 6 (1991) 2613 [INSPIRE].

[62] P.H. Ginsparg, Matrix models of 2-D gravity, hep-th/9112013 [INSPIRE].

[63] M. Gross, Tensor models and simplicial quantum gravity in $>2-D$, Nucl. Phys. Proc. Suppl. 25A (1992) 144 [INSPIRE].

[64] D.V. Boulatov, A model of three-dimensional lattice gravity, Mod. Phys. Lett. A 7 (1992) 1629 [hep-th/9202074] [INSPIRE].

[65] L. Freidel, Group field theory: An Overview, Int. J. Theor. Phys. 44 (2005) 1769 [hep-th/0505016] [INSPIRE].

[66] D. Oriti, Quantum gravity as a quantum field theory of simplicial geometry, gr-qc/0512103 [INSPIRE].

[67] D. Oriti, The group field theory approach to quantum gravity, gr-qc/0607032 [INSPIRE].

[68] J. Magnen, K. Noui, V. Rivasseau and M. Smerlak, Scaling behaviour of three-dimensional group field theory, Class. Quant. Grav. 26 (2009) 185012 [arXiv:0906.5477] [INSPIRE].

[69] L. Freidel, R. Gurau and D. Oriti, Group field theory renormalization - the $3 d$ case: power counting of divergences, Phys. Rev. D 80 (2009) 044007 [arXiv: 0905.3772] [INSPIRE].

[70] E.T. Akhmedov, Towards the theory of non-Abelian tensor fields. I., Theor. Math. Phys. 145 (2005) 1646 [hep-th/0503234] [INSPIRE].

[71] E.T. Akhmedov, V. Dolotin and A. Morozov, Comment on the surface exponential for tensor fields, JETP Lett. 81 (2005) 639 [hep-th/0504160] [INSPIRE].

[72] R. Gurau, Colored Group Field Theory, Commun. Math. Phys. 304 (2011) 69 [arXiv:0907.2582] [INSPIRE].

[73] R. Gurau, Topological Graph Polynomials in Colored Group Field Theory, Annales Henri Poincaré 11 (2010) 565 [arXiv: 0911.1945] [INSPIRE].

[74] R. Gurau, Lost in Translation: Topological Singularities in Group Field Theory, Class. Quant. Grav. 27 (2010) 235023 [arXiv:1006.0714] [INSPIRE].

[75] R. Gurau, The complete $1 / N$ expansion of colored tensor models in arbitrary dimension, Annales Henri Poincaré 13 (2012) 399 [arXiv:1102.5759] [INSPIRE].

[76] J. Ben Geloun, R. Gurau and V. Rivasseau, EPRL/FK Group Field Theory, Europhys. Lett. 92 (2010) 60008 [arXiv: 1008.0354] [InSPIRE].

[77] R. Gurau and V. Rivasseau, The $1 / N$ expansion of colored tensor models in arbitrary dimension, Europhys. Lett. 95 (2011) 50004 [arXiv:1101.4182] [INSPIRE].

[78] R. Gurau and J.P. Ryan, Colored Tensor Models - a review, SIGMA 8 (2012) 020 [arXiv: 1109.4812] [INSPIRE]. 
[79] V. Bonzom, R. Gurau and V. Rivasseau, Random tensor models in the large- $N$ limit: Uncoloring the colored tensor models, Phys. Rev. D 85 (2012) 084037 [arXiv:1202.3637] [INSPIRE].

[80] R. Gurau et al., Special Issue on Tensor Models, Formalism and Applications, SIGMA 12 (2016) [http://www.emis.de/journals/SIGMA/Tensor_Models.html].

[81] V. Bonzom, R. Gurau, A. Riello and V. Rivasseau, Critical behavior of colored tensor models in the large-N limit, Nucl. Phys. B 853 (2011) 174 [arXiv:1105.3122] [INSPIRE].

[82] R. Gurau, A generalization of the Virasoro algebra to arbitrary dimensions, Nucl. Phys. B 852 (2011) 592 [arXiv:1105.6072] [INSPIRE].

[83] R. Gurau, The Schwinger Dyson equations and the algebra of constraints of random tensor models at all orders, Nucl. Phys. B 865 (2012) 133 [arXiv:1203.4965] [InSPIRE].

[84] V. Bonzom, Revisiting random tensor models at large- $N$ via the Schwinger-Dyson equations, JHEP 03 (2013) 160 [arXiv: 1208.6216] [INSPIRE].

[85] V. Bonzom, New 1/N expansions in random tensor models, JHEP 06 (2013) 062 [arXiv:1211.1657] [INSPIRE].

[86] V. Bonzom and F. Combes, Tensor models from the viewpoint of matrix models: the case of loop models on random surfaces, arXiv:1304.4152 [INSPIRE].

[87] V. Bonzom, R. Gurau, J.P. Ryan and A. Tanasa, The double scaling limit of random tensor models, JHEP 09 (2014) 051 [arXiv: 1404.7517] [INSPIRE].

[88] R. Gurau, A. Tanasa and D.R. Youmans, The double scaling limit of the multi-orientable tensor model, Europhys. Lett. 111 (2015) 21002 [arXiv: 1505.00586] [INSPIRE].

[89] A. Tanasa, Multi-orientable Group Field Theory, J. Phys. A 45 (2012) 165401 [arXiv:1109.0694] [INSPIRE].

[90] A. Tanasa, The Multi-Orientable Random Tensor Model, a Review, SIGMA 12 (2016) 056 [arXiv: 1512.02087] [INSPIRE].

[91] S. Dartois, V. Rivasseau and A. Tanasa, The $1 / N$ expansion of multi-orientable random tensor models, Annales Henri Poincaré 15 (2014) 965 [arXiv:1301.1535] [INSPIRE].

[92] D. Garner and S. Ramgoolam, Holographic Hierarchy in the Gaussian Matrix Model via the Fuzzy Sphere, Nucl. Phys. B 875 (2013) 244 [arXiv:1303.3246] [INSPIRE].

[93] J. Ben Geloun and S. Ramgoolam, Counting Tensor Model Observables and Branched Covers of the 2-Sphere, arXiv:1307.6490 [INSPIRE].

[94] P. Cristofori, E. Fominykh, M. Mulazzani and V. Tarkaev, 4-colored graphs and knot/link complements, arXiv:1609.02357.

[95] D. Benedetti et al., Proceedings of the 2nd French-Russian Conference on Random Geometry and Physics, Institut Henri Poincaré, Paris, 17-21 October 2016, http://www.th.u-psud.fr/RGP16/.

[96] J.M. Daul, V.A. Kazakov and I.K. Kostov, Rational theories of 2-D gravity from the two matrix model, Nucl. Phys. B 409 (1993) 311 [hep-th/9303093] [INSPIRE].

[97] M. Anazawa, A. Ishikawa and H. Itoyama, Macroscopic three loop amplitudes from the two matrix model, Phys. Lett. B 362 (1995) 59 [hep-th/9508009] [INSPIRE]. 
[98] M. Anazawa and H. Itoyama, Macroscopic n loop amplitude for minimal models coupled to two-dimensional gravity: Fusion rules and interactions, Nucl. Phys. B 471 (1996) 334 [hep-th/9511220] [INSPIRE].

[99] P. Zinn-Justin, Some Matrix Integrals related to Knots and Links, math-ph/9910010.

[100] A. Gerasimov, A. Morozov and K. Selivanov, Bogolyubov's recursion and integrability of effective actions, Int. J. Mod. Phys. A 16 (2001) 1531 [hep-th/0005053] [inSPIRE].

[101] K. Norton and G.A. Jaroszkiewicz, Principles of discrete time mechanics: 3. Quantum field theory, J. Phys. A 31 (1998) 977 [hep-th/9707029] [InSPIRE].

[102] D. Kreimer, On overlapping divergences, Commun. Math. Phys. 204 (1999) 669 [hep-th/9810022] [INSPIRE].

[103] A. Connes and D. Kreimer, Hopf algebras, renormalization and noncommutative geometry, Commun. Math. Phys. 199 (1998) 203 [hep-th/9808042] [INSPIRE].

[104] A. Connes and D. Kreimer, Lessons from quantum field theory: Hopf algebras and space-time geometries, Lett. Math. Phys. 48 (1999) 85 [hep-th/9904044] [INSPIRE].

[105] A. Connes and D. Kreimer, Renormalization in quantum field theory and the Riemann-Hilbert problem, JHEP 09 (1999) 024 [hep-th/9909126] [INSPIRE].

[106] A. Connes and D. Kreimer, Renormalization in quantum field theory and the Riemann-Hilbert problem. 1. The Hopf algebra structure of graphs and the main theorem, Commun. Math. Phys. 210 (2000) 249 [hep-th/9912092] [INSPIRE].

[107] A. Connes and D. Kreimer, Renormalization in quantum field theory and the Riemann-Hilbert problem. 2. The $\beta$-function, diffeomorphisms and the renormalization group, Commun. Math. Phys. 216 (2001) 215 [hep-th/0003188] [INSPIRE].

[108] I. Gelfand, M. Kapranov and A. Zelevinsky, Discriminants, Resultants and Multidimensional Determinants, Birkhauser (1994).

[109] I. Gelfand, M. Kapranov and A. Zelevinsky, Discriminants of polynomials in several variables and triangulations of Newton polyhedra, Leningrad Math. J. 2 (1991) 499.

[110] V. Dolotin and A. Morozov, Introduction to Non-Linear Algebra, hep-th/0609022 [INSPIRE].

[111] A. Morozov and S. Shakirov, New and Old Results in Resultant Theory, Theor. Math. Phys. 163 (2010) 587 [arXiv:0911.5278] [InSPIRE].

[112] A.S. Alexandrov, A. Mironov and A. Morozov, Partition functions of matrix models as the first special functions of string theory. 1. Finite size Hermitean one matrix model, Int. J. Mod. Phys. A 19 (2004) 4127 [hep-th/0310113] [INSPIRE].

[113] F.J. Dyson, Statistical theory of the energy levels of complex systems. I, J. Math. Phys. 3 (1962) 140 [INSPIRE].

[114] A. Alexandrov, A. Mironov and A. Morozov, BGWM as Second Constituent of Complex Matrix Model, JHEP 12 (2009) 053 [arXiv:0906.3305] [INSPIRE].

[115] A.S. Alexandrov, A. Mironov and A. Morozov, Instantons and merons in matrix models, Physica D 235 (2007) 126 [hep-th/0608228] [INSPIRE].

[116] A. Alexandrov, A. Mironov and A. Morozov, BGWM as Second Constituent of Complex Matrix Model, JHEP 12 (2009) 053 [arXiv: 0906.3305] [INSPIRE]. 
[117] B. Eynard and N. Orantin, Invariants of algebraic curves and topological expansion, Commun. Num. Theor. Phys. 1 (2007) 347 [math-ph/0702045] [INSPIRE].

[118] N. Orantin, Symplectic invariants, Virasoro constraints and Givental decomposition, arXiv:0808.0635 [INSPIRE].

[119] R. Dijkgraaf and C. Vafa, Matrix models, topological strings and supersymmetric gauge theories, Nucl. Phys. B 644 (2002) 3 [hep-th/0206255] [INSPIRE].

[120] R. Dijkgraaf and C. Vafa, On geometry and matrix models, Nucl. Phys. B 644 (2002) 21 [hep-th/0207106] [INSPIRE].

[121] R. Dijkgraaf and C. Vafa, A perturbative window into nonperturbative physics, hep-th/0208048 [INSPIRE].

[122] L. Chekhov and A. Mironov, Matrix models versus Seiberg-Witten/Whitham theories, Phys. Lett. B 552 (2003) 293 [hep-th/0209085] [INSPIRE].

[123] L. Chekhov, A. Marshakov, A. Mironov and D. Vasiliev, DV and WDVV, Phys. Lett. B 562 (2003) 323 [hep-th/0301071] [INSPIRE].

[124] L. Chekhov, A. Marshakov, A. Mironov and D. Vasiliev, Complex geometry of matrix models, Proc. Steklov Inst. Math. 251 (2005) 254 [hep-th/0506075] [INSPIRE].

[125] R. Dijkgraaf, S. Gukov, V.A. Kazakov and C. Vafa, Perturbative analysis of gauged matrix models, Phys. Rev. D 68 (2003) 045007 [hep-th/0210238] [INSPIRE].

[126] V.A. Kazakov and A. Marshakov, Complex curve of the two matrix model and its tau function, J. Phys. A 36 (2003) 3107 [hep-th/0211236] [INSPIRE].

[127] H. Itoyama and A. Morozov, The Dijkgraaf-Vafa prepotential in the context of general Seiberg-Witten theory, Nucl. Phys. B 657 (2003) 53 [hep-th/0211245] [InSPIRE].

[128] H. Itoyama and A. Morozov, Experiments with the WDVV equations for the gluino condensate prepotential: The Cubic (two cut) case, Phys. Lett. B 555 (2003) 287 [hep-th/0211259] [INSPIRE].

[129] H. Itoyama and A. Morozov, Calculating gluino condensate prepotential, Prog. Theor. Phys. 109 (2003) 433 [hep-th/0212032] [INSPIRE].

[130] H. Itoyama and A. Morozov, Gluino condensate (CIV-DV) prepotential from its Whitham time derivatives, Int. J. Mod. Phys. A 18 (2003) 5889 [hep-th/0301136] [InSPIRE].

[131] H. Itoyama and H. Kanno, Supereigenvalue model and Dijkgraaf-Vafa proposal, Phys. Lett. B 573 (2003) 227 [hep-th/0304184] [INSPIRE].

[132] H. Itoyama and H. Kanno, Whitham prepotential and superpotential, Nucl. Phys. B 686 (2004) 155 [hep-th/0312306] [INSPIRE].

[133] G. Bonelli, L. Bonora and A. Ricco, Conifold geometries, topological strings and multi-matrix models, Phys. Rev. D 72 (2005) 086001 [hep-th/0507224] [INSPIRE].

[134] G. Bonelli, L. Bonora and A. Ricco, Conifold geometries, matrix models and quantum solutions, hep-th/0511152 [INSPIRE].

[135] A. Mironov, Matrix models vs. matrix integrals, Theor. Math. Phys. 146 (2006) 63 [hep-th/0506158] [INSPIRE].

[136] A. Morozov and S. Shakirov, Generation of Matrix Models by W-operators, JHEP 04 (2009) 064 [arXiv:0902.2627] [INSPIRE]. 
[137] A. Alexandrov, Cut-and-Join operator representation for Kontsewich-Witten tau-function, Mod. Phys. Lett. A 26 (2011) 2193 [arXiv: 1009.4887] [InSPIRE].

[138] A. Alexandrov, Open intersection numbers, Kontsevich-Penner model and cut-and-join operators, JHEP 08 (2015) 028 [arXiv:1412.3772] [INSPIRE].

[139] A. Alexandrov, Cut-and-join description of generalized Brezin-Gross-Witten model, arXiv: 1608.01627 [INSPIRE].

[140] A. Alexandrov, A. Mironov, A. Morozov and S. Natanzon, On KP-integrable Hurwitz functions, JHEP 11 (2014) 080 [arXiv:1405.1395] [inSPIRE].

[141] A. Mironov, A. Morozov and S. Natanzon, Complete Set of Cut-and-Join Operators in Hurwitz-Kontsevich Theory, Theor. Math. Phys. 166 (2011) 1 [arXiv:0904.4227] [INSPIRE].

[142] A. Mironov, A. Morozov and S. Natanzon, Algebra of differential operators associated with Young diagrams, J. Geom. Phys. 62 (2012) 148 [arXiv:1012.0433] [INSPIRE].

[143] A. Okounkov, Generating functions for intersection numbers on moduli spaces of curves, math/0101201 [INSPIRE].

[144] E. Brézin and S. Hikami, Intersection numbers of Riemann surfaces from Gaussian matrix models, JHEP 10 (2007) 096 [arXiv: 0709. 3378] [INSPIRE].

[145] E. Brézin and S. Hikami, Intersection theory from duality and replica, Commun. Math. Phys. 283 (2008) 507 [arXiv: 0708.2210] [INSPIRE].

[146] E. Brézin and S. Hikami Level Spacing of Random Matrices in an External Source, Phys. Rev. E 58 (1998) 7176 [cond-mat/9804024].

[147] A.S. Alexandrov, A. Mironov, A. Morozov and P. Putrov, Partition Functions of Matrix Models as the First Special Functions of String Theory. II. Kontsevich Model, Int. J. Mod. Phys. A 24 (2009) 4939 [arXiv:0811.2825] [InSPIRE].

[148] A. Morozov, Faces of matrix models, JETP Lett. 95 (2012) 586 [arXiv:1204.3953] [INSPIRE].

[149] J. Harer and D. Zagier, The Euler characteristic of the moduli space of curves, Invent. Math. 85 (1986) 457.

[150] C. Itzykson and J.B. Zuber, Matrix Integration and Combinatorics of Modular Groups, Commun. Math. Phys. 134 (1990) 197 [InSPIRE].

[151] S.K. Lando and A.K. Zvonkin, Embedded graphs, Max-Plank-Institut für Mathematik, Preprint Series 2001 (63).

[152] A. Morozov and S. Shakirov, Exact 2-point function in Hermitian matrix model, JHEP 12 (2009) 003 [arXiv: 0906.0036] [INSPIRE].

[153] A. Morozov and S. Shakirov, From Brezin-Hikami to Harer-Zagier formulas for Gaussian correlators, arXiv:1007.4100 [INSPIRE].

[154] W. Fulton, Young tableaux: with applications to representation theory and geometry, LMS (1997).

[155] A. Gerasimov, A. Marshakov, A. Mironov, A. Morozov and A. Orlov, Matrix models of $2-D$ gravity and Toda theory, Nucl. Phys. B 357 (1991) 565 [INSPIRE]. 
[156] S. Kharchev, A. Marshakov, A. Mironov, A. Orlov and A. Zabrodin, Matrix models among integrable theories: Forced hierarchies and operator formalism, Nucl. Phys. B 366 (1991) 569 [INSPIRE].

[157] S. Kharchev, A. Marshakov, A. Mironov and A. Morozov, Generalized Kontsevich model versus Toda hierarchy and discrete matrix models, Nucl. Phys. B 397 (1993) 339 [hep-th/9203043] [INSPIRE].

[158] S. Kharchev, A. Marshakov, A. Mironov and A. Morozov, Generalized Kazakov-Migdal-Kontsevich model: Group theory aspects, Int. J. Mod. Phys. A 10 (1995) 2015 [hep-th/9312210] [inSPIRE].

[159] A. Alexandrov, A. Mironov, A. Morozov and S. Natanzon, Integrability of Hurwitz Partition Functions. I. Summary, J. Phys. A 45 (2012) 045209 [arXiv:1103.4100] [InSPIRE].

[160] A. Orlov and D.M. Shcherbin, Hypergeometric solutions of soliton equations, Theor. Math. Phys. 128 (2001) 906.

[161] A. Orlov, Hypergeometric functions as in?nite-soliton Tau functions, Theor. Math. Phys. 146 (2006) 183.

[162] A. Mironov, A. Morozov and Z. Zakirova, Comment on integrability in Dijkgraaf-Vafa beta-ensembles, Phys. Lett. B 711 (2012) 332 [arXiv:1202.6029] [INSPIRE].

[163] A. Mironov and A. Morozov, On the complete perturbative solution of one-matrix models, arXiv: 1705.00976 [INSPIRE].

[164] M. Rosso and V.F.R. Jones, On the invariants of torus knots derived from quantum groups, J. Knot Theory Ramifications 2 (1993) 97.

[165] X.-S. Lin and H. Zheng, On the Hecke algebras and the colored HOMFLY polynomial, Trans. Am. Math. Soc. 362 (2010) 1 [math/0601267].

[166] P. Dunin-Barkowski, A. Mironov, A. Morozov, A. Sleptsov and A. Smirnov, Superpolynomials for toric knots from evolution induced by cut-and-join operators, JHEP 03 (2013) 021 [arXiv: 1106.4305] [INSPIRE].

[167] A. Mironov, A. Morozov and An. Morozov, Character expansion for HOMFLY polynomials. I. Integrability and difference equations, in Strings, Gauge Fields, and the Geometry Behind: The Legacy of Maximilian Kreuzer, A. Rebhan et al. eds., World Scietific Publishins Co.Pte.Ltd. (2013), pg.101-118 [arXiv: 1112.5754].

[168] A. Mironov, A. Morozov and A. Morozov, Character expansion for HOMFLY polynomials. II. Fundamental representation. Up to five strands in braid, JHEP 03 (2012) 034 [arXiv:1112.2654] [INSPIRE].

[169] L. Chekhov and Yu. Makeenko, A Hint on the external field problem for matrix models, Phys. Lett. B 278 (1992) 271 [hep-th/9202006] [INSPIRE].

[170] T.R. Morris, Checkered surfaces and complex matrices, Nucl. Phys. B 356 (1991) 703 [INSPIRE].

[171] Yu. Makeenko, Complex-matrix model and 2D quantum gravity, Pis'ma Zh. Eksp. Teor. Fiz. 52 (1990) 885.

[172] Yu. Makeenko, A. Marshakov, A. Mironov and A. Morozov, Continuum versus discrete Virasoro in one matrix models, Nucl. Phys. B 356 (1991) 574 [INSPIRE]. 
[173] V. Rivasseau, Loop Vertex Expansion for Higher Order Interactions, arXiv:1702.07602 [INSPIRE].

[174] M. Raasakka and A. Tanasa, Combinatorial Hopf algebra for the Ben Geloun-Rivasseau tensor field theory, arXiv:1306.1022 [INSPIRE].

[175] R.C. Avohou, V. Rivasseau and A. Tanasa, Renormalization and Hopf algebraic structure of the five-dimensional quartic tensor field theory, J. Phys. A 48 (2015) 485204 [arXiv: 1507.03548] [INSPIRE].

[176] A. Connes and H. Moscovici, Hopf Algebras, Cyclic Cohomology and the Transverse Index Theorem, Commun. Math. Phys. 198 (1998) 199.

[177] Aristotle, Meteorology, english translation by E.W. Webster, http://classics.mit.edu//Aristotle/meteorology.html.

[178] R.L. Lee and A.B. Fraser, The Rainbow Bridge: Rainbows in Art, Myth, and Science, The Pennsylvania State University Press (2001) [ISBN: 978-0-271-01977-2].

[179] A. Mironov, A. Morozov, A. Morozov, P. Ramadevi and V.K. Singh, Colored HOMFLY polynomials of knots presented as double fat diagrams, JHEP 07 (2015) 109 [arXiv: 1504.00371] [INSPIRE].

[180] A. Mironov and A. Morozov, Towards effective topological field theory for knots, Nucl. Phys. B 899 (2015) 395 [arXiv:1506.00339] [INSPIRE].

[181] A. Mironov, A. Morozov, A. Morozov, P. Ramadevi, V.K. Singh and A. Sleptsov, Tabulating knot polynomials for arborescent knots, J. Phys. A 50 (2017) 085201 [arXiv: 1601.04199] [INSPIRE].

[182] A.V. Zabrodin, Nonarchimedean Strings and Bruhat-tits Trees, Commun. Math. Phys. 123 (1989) 463 [INSPIRE].

[183] L.O. Chekhov, A.D. Mironov and A.V. Zabrodin, Multiloop Calculations in p-adic String Theory and Bruhat-tits Trees, Commun. Math. Phys. 125 (1989) 675 [INSPIRE].

[184] V. Bonzom and S. Dartois, Blobbed topological recursion for the quartic melonic tensor model, arXiv:1612.04624 [INSPIRE]. 